Aus der Abteilung Psychiatrie und Psychotherapie

(Komm. Direktor: Prof. Dr. B. Bandelow)

im Zentrum Psychosoziale Medizin

der Medizinischen Fakultät der Universität Göttingen

\title{
Einfluss eines videobasierten Furchtappells auf Abbrüche im stationären Drogenentzug
}

Inaugural - Dissertation

zur Erlangung des Doktorgrades der Medizinischen Fakultät der Georg-August-Universität zu Göttingen

vorgelegt von

Dorothee Pletke

aus Lüchow

Göttingen 2013 
Dekan:

I. Berichterstatter:

II. Berichterstatter/-in:

III. Berichterstatter/-in:

Tag der mündlichen Prüfung:
Prof. Dr. rer. nat. H. K. Kroemer

Prof. Dr. med. W. Poser

Prof. Dipl.-Psych. Dr. rer. nat. E. Leibing

Prof. Dr. med. A. Rothenberger

01.07 .2015 


\section{Inhaltsverzeichnis}

1

Einleitung 1

1.1 Theoretische und empirische Grundlagen zum stationären

Drogenentzug und zum Einsatz von Furchtappellen

1.1.1 Epidemiologische und gesundheitsökonomische Aspekte der Suchterkrankungen

1.1.2 Behandlungsabbrüche im stationären Drogenentzug sowie Behandlungsstrategien

1.1.3 Einflussfaktoren auf Behandlungsabbrüche im stationären Drogenentzug.

1.2 Die Problematik der Diagnostik komorbider psychiatrischer Störungen im Rahmen des stationären Entzugs illegaler Drogen ....11

1.2.1 Empirische Befunde zur Häufigkeit komorbider psychiatrischer Störungen und zur Suizidalität bei Drogenabhängigkeit.

1.2.2 Empirische Befunde zum Einfluss komorbider psychiatrischer Störungen auf die Behandlung einer Drogenabhängigkeit.

1.2.3 Geschlechtsspezifische Besonderheiten drogenabhängiger Patienten mit komorbiden psychiatrischen Störungen.

1.3 Zur Geschichte der methadongestützten Behandlung der Opiatabhängigkeit

1.4 Übersicht der Literatur zum Einsatz der Videografie in der Psychiatrie und Psychotherapie .29

1.5 Furchtappelle als Mittel zur Einstellungs- und Verhaltensänderung.....34

1.5.1 Die Rolle der Informationsverarbeitung im Rahmen eines Furchtappells. .37

1.5.2 Der Furchtappell als Mittel zur Suchtprävention ............................42

1.6 Ableitung der klinischen Hypothesen zur Abbruchquote .....................45

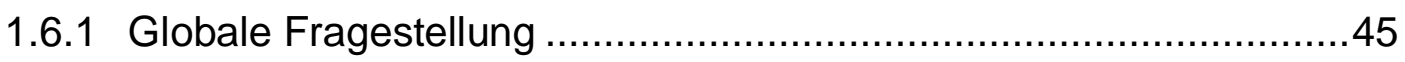

1.6.2 Klinische Erfahrungen aus dem Bereich der Entgiftungsbehandlung.

1.6.3 Mögliche Bedeutung eines Furchtappells in der Entgiftung... .46

2 Methoden

2.1 Der Furchtappell 50

2.1.1 Setting der Videodokumentation .51

2.1.2 Situation der Videokonfrontation .52

2.1.3 Procedere bei Abbruchwunsch von Kontrollpersonen .55 
2.2 Fragebogen zur Erfassung der inneren Verarbeitung …....................56

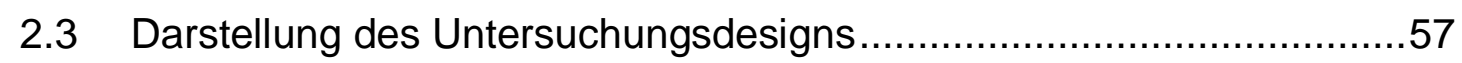

2.4 Darstellung der experimentellen Variation/ Gruppenbildung ................58

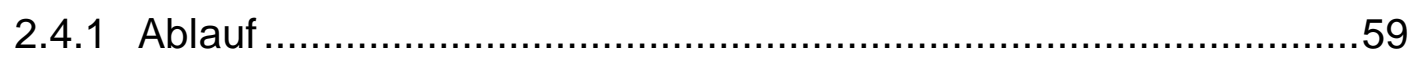

2.5 Darstellung des multimodalen Therapiekonzepts auf der

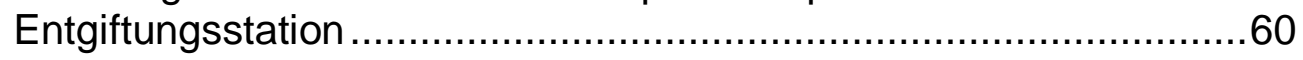

2.5.1 Medikamentöse Entgiftungsschemata:........................................60

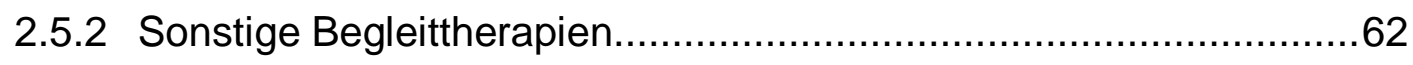

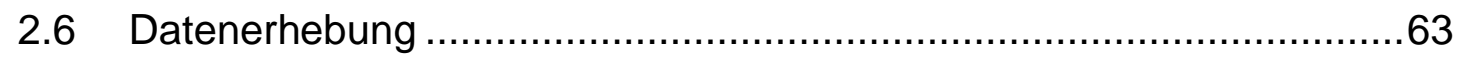

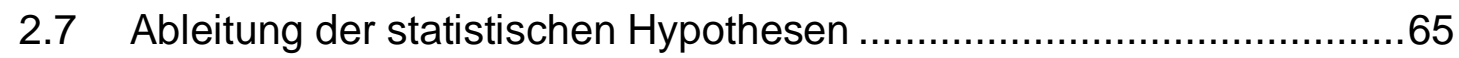

2.7.1 Variablen und angenommene Wirkungszusammenhänge im

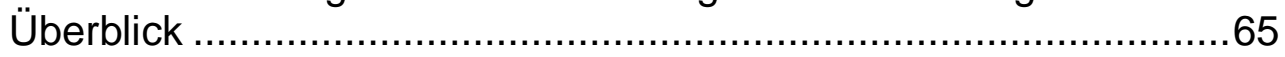

2.7.2 Zuordnung der Variablen und Operationalisierung ........................66

2.7.3 Ableitung der klinischen Vorhersagen und statistischen

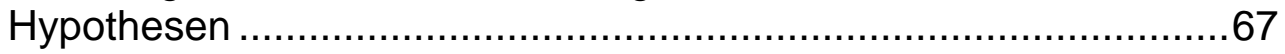

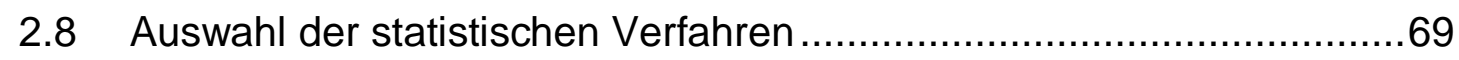

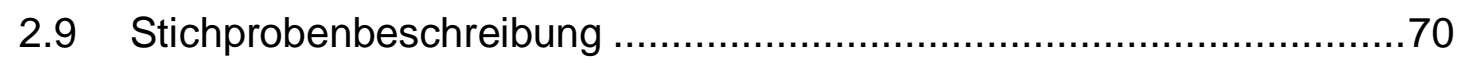

2.9.1 Darstellung der im Untersuchungszeitraum stationär aufgenommenen Patienten, die nicht in die Studie eingeschlossen werden konnten.

3 Ergebnisse .75

3.1 Experimentelle Variation des Furchtappells .....................................75

3.2 Der Fragebogen zur Erfassung der inneren Verarbeitung …...............77

3.3 Statistische Hypothesenprüfungen.................................................

3.4 Subjektiv eingeschätzter Nutzen des verwendeten Furchtappells .......91

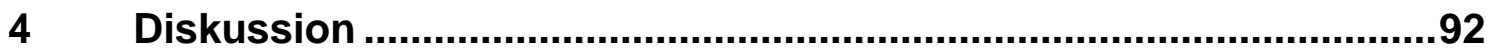

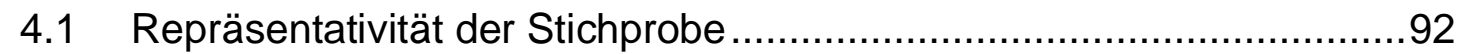

4.2 Diskussion zum Einfluss des videobasierten Furchtappells auf die Abbruchquote im stationären Drogenentzug ..................................94

4.3 Diskussion zum Zusammenhang zwischen innerer Verarbeitung des Furchtappells und dem Durchhaltevermögen bzw. Abbruchverhalten im stationären Drogenentzug

4.4 Diskussion zur geschlechtsspezifischen Wirkung von Furchtappellen103 
6.1 Anhang A Tabellen 20-27 ....

6.2 Anhang B Patienteneinverständniserklärungen und

Patientenaufklärungen.. 120

6.2.1 Patienteneinverständniserklärung zur Studie 120

6.2.2 Patientenaufklärung zur Studie 121

6.2.3 Patientenaufklärung zur Studie (Kontrollgruppe) 122

6.2.4 Patienteneinverständniserklärung zur Studie (Kontrollgruppe)...... 123

6.3 Anhang C Erhebungsbögen 124

$7 \quad$ Literaturverzeichnis 129 


\section{Abbildungsverzeichnis}

Abbildung 1: Zielpyramide der Drogenentzugsbehandlung mit niederschwelligem Zugangsweg

Abbildung 2: Zielsetzungen in der Suchttherapie jenseits des

Abstinenzparadigmas .26

Abbildung 3: Dreikomponentenmodell der Einstellung nach Rosenberg und Hovland .35

Abbildung 4: Prozesse der Informatonsverarbeitung. .35

Abbildung 5: Mögliche Antworten auf den Werbespot „Just say no“ .36

Abbildung 6: Revidierte Theorie der Schutzmotivation von Rogers 1983

Abbildung 7: Darstellung des Arztzimmers .52

Abbildung 8: Fragebogen nach Ansicht des Videos 57

Abbildung 9: Darstellung der untersuchten Variablen bzw. Merkmale mit den jeweils in der vorliegenden Studie betrachteten (Wirkungs-)

Zusammenhängen .65

Abbildung 10: Versuchsplan - Aufteilung der Gruppen im Überblick .66

Abbildung 11: Anzahl der Abbrüche in der Kontroll- und Experimentalgruppe 80 Abbildung 12: Anzahl der Videokonfrontationen bei Männern und Frauen .83

Abbildung 13: Darstellung der Anzahl der Nennungen von

Antwortalternativen zur Frage nach dem Suchtdruck infolge der Videokonfrontation .86 


\section{Tabellenverzeichnis}

Tabelle 1: Abbruchquoten bei stationären Entgiftungen................................ 4

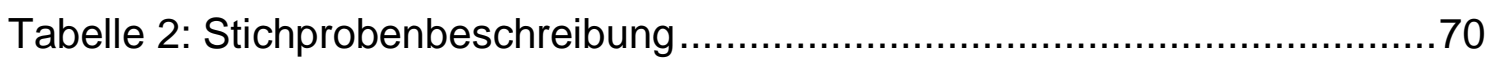

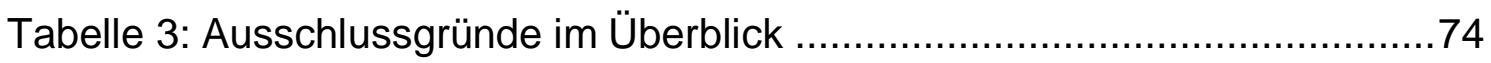

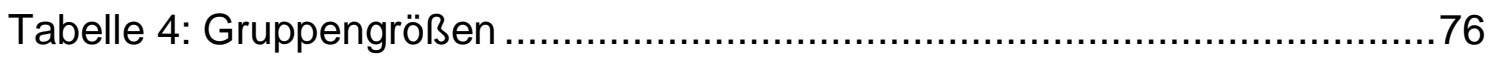

Tabelle 5: Deskriptive Statistiken zur inneren Verarbeitung ..............................79

Tabelle 6: Anzahl der Abbrüche in Kontroll- und Experimentalgruppe ...............80

Tabelle 7: Darstellung der Subgruppen der Experimentalgruppe.......................81

Tabelle 8: Darstellung der Abbruchhäufigkeit in der Subgruppe mit

Videokonfrontation und der Kontrollgruppe ................................... 82

Tabelle 9: Abhängigkeit der Bereitschaft zur Videokonfrontation vom

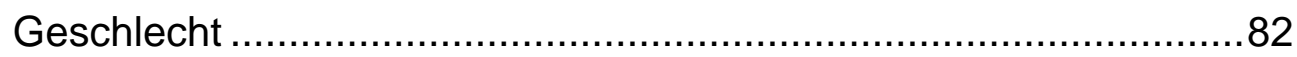

Tabelle 10: Zusammenhang zwischen Behandlungsabbruch und Ausmaß der inneren Berührung durch die Videokonfrontation ............................84

Tabelle 11: Darstellung des Zusammenhangs zwischen Ausmaß der

Reaktanz und dem Durchhaltevermögen in der stationären

Entgiftung

Tabelle 12: Darstellung des Zusammenhangs zwischen erzeugtem

Suchtdruck und Durchhaltevermögen der Patienten

Tabelle 13: Darstellung des Zusammenhangs zwischen Geschlecht und innerer Verarbeitung des Videos

Tabelle 14: Darstellung des Zusammenhangs zwischen Geschlecht und

Reaktanz .88

Tabelle 15: Darstellung des Zusammenhangs zwischen Geschlecht und

Suchtdruck .89

Tabelle 16: Darstellung der Zusammenhänge zwischen Geschlecht und Abbruch für die Subgruppe mit Videokonfrontation .90

Tabelle 17: Darstellung der Zusammenhänge zwischen Geschlecht und Abbruch für die Subgruppe ohne Videodokumentation (Kontrollgruppe). .90 
Tabelle 18: Darstellung der Antworten auf die Frage nach einem standardmäßigen Einsatz des videobasierten Furchtappells im stationären Drogenentzug .

Tabelle 19: Einschätzung des Nutzens des Furchtappells bei Männern und

Frauen. .91

Tabelle 20: Korrelationsmatrix 112

Tabelle 21: Kommunalitäten. 113

Tabelle 22: Erklärte Gesamtvarianz 113

Tabelle 23: Komponentenmatrix(a) 114

Tabelle 24: Rotierte Komponentenmatrix(a) 114

Tabelle 25: Komponententransformationsmatrix 115

Tabelle 26: Zusammenhang von Traumatisierung und Suchterkrankung. 116

Tabelle 27: Zusammenhang von Traumatisierung, Posttraumatischer Belastungsstörung und Substanzmittel-Konsum. 


\section{Verwendete Abkürzungen}

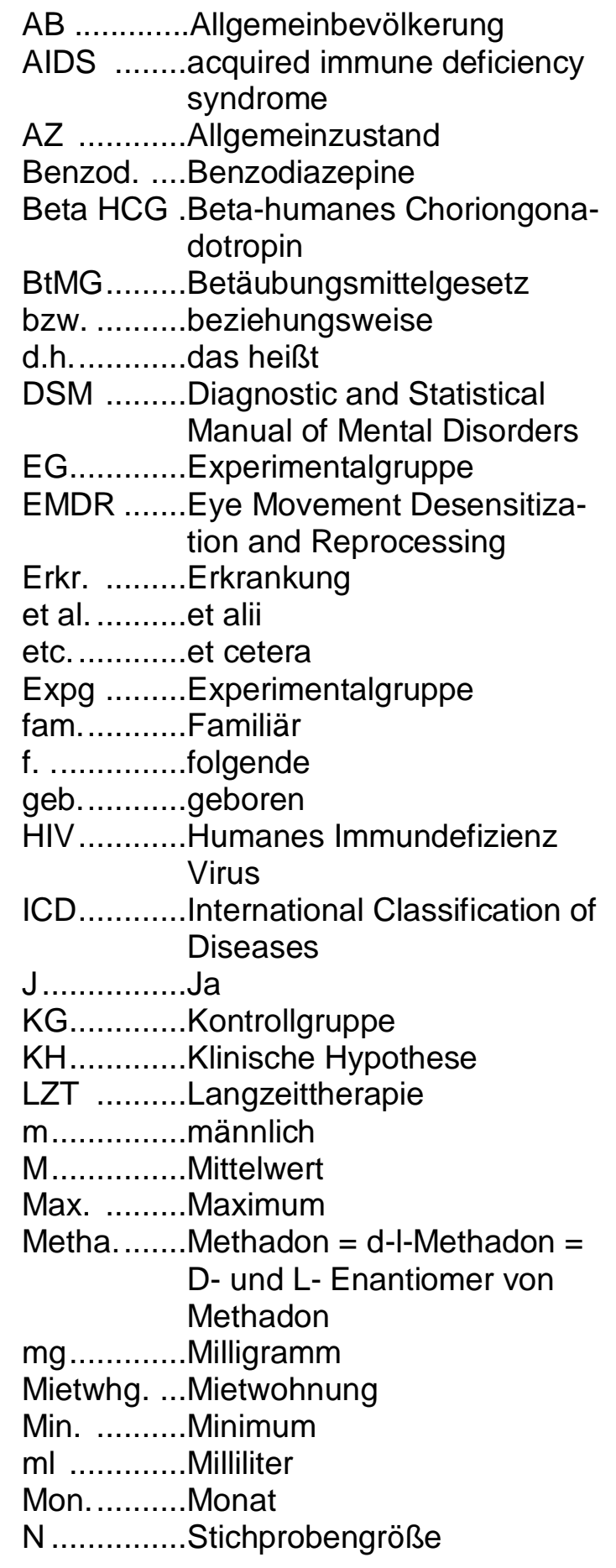

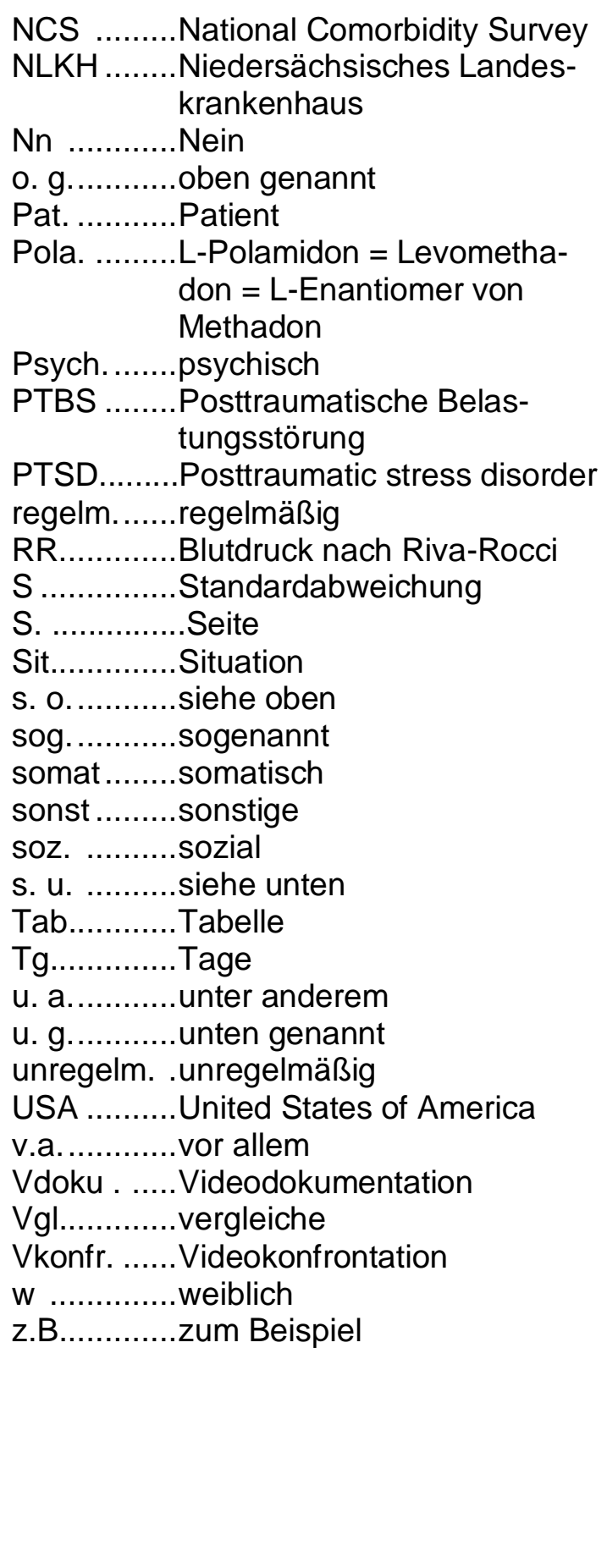


Im Asklepios Fachklinikum Göttingen, das zum Zeitpunkt der im Folgenden dargestellten Untersuchung noch in Trägerschaft des Landes Niedersachsen war, und damals Niedersächsischen Landeskrankenhaus Göttingen hieß, gibt es Spezialstationen zur Entgiftung illegaler Drogen seit Anfang 1994. Obwohl auf den Stationen schon damals entsprechend innovativster Konzepte eine warme Methadongestützte Entgiftung angeboten wurde, sind die Mitarbeiter fortgesetzt mit einer erheblichen Abbruchquote bei den Entgiftungsbehandlungen konfrontiert. Misserfolge in bis zu über 50\% der Fälle wirken sowohl demotivierend auf das Team als auch auf die Patientengruppe. Daher wurde und wird in Göttingen weiterhin nach Möglichkeiten gesucht, die Haltequote im stationären Drogenentzug zu verbessern.

Über Jahre hinweg wurde zu diesem Zweck unter anderem ein videobasierter Furchtappell in schwierigen Phasen der Entgiftung eingesetzt: Wenn die Entzugssymptome am stärksten sind, wird den Patienten ihr ursprünglicher Allgemeinzustand mittels einer Videoaufzeichnung vergegenwärtigt und die Gefahren eines Abbruchs werden aufgezeigt. Begleitet wird dies durch ein motivierendes therapeutisches Gespräch. Insgesamt soll es den Patienten dadurch erleichtert werden, die Entgiftungsbehandlung weiter durchzuhalten.

Der notwendigen Videodokumentation bei der stationären Aufnahme sowie der späteren Konfrontationssitzung kommt ein nicht unerheblicher Arbeitsaufwand zu. Der praktische Nutzen dieses Vorgehens soll daher nun in der vorliegenden Arbeit geprüft, mögliche Wirkmechanismen systematisch untersucht und eventuelle Veränderungsmöglichkeiten bzw. -notwendigkeiten sollen aufgezeigt werden. Um aussagekräftige Ergebnisse zu erzielen, ist es wichtig, die empirische Prüfung direkt im gegebenen Anwendungsfeld vorzunehmen. Das stationäre Setting sowie auch die Behandlungsklientel weisen spezifische Besonderheiten auf, denen eine laborgestützte Untersuchung nicht gerecht werden könnte. 


\title{
1.1
}

\author{
Theoretische und empirische Grundlagen zum statio- \\ nären Drogenentzug und zum Einsatz von Furchtappel- \\ Ien
}

Da die empirische Prüfung des videobasierten Furchtappells wie beschrieben direkt im Anwendungsfeld erfolgen soll, werden zunächst die Besonderheiten dieses speziellen Settings verdeutlicht: Epidemiologische und gesundheitsökonomische Aspekte der Suchterkrankungen werden benannt, empirische Grundlagen zu Behandlungsabbrüchen und psychiatrischen Komorbiditäten im stationären Drogenentzug werden aufgeführt und es wird auf geschlechtsspezifische Besonderheiten hingewiesen. Daran anschließen werden sich eine Darstellung der Geschichte der Methadon-gestützten Entgiftungsbehandlung sowie eine Literaturübersicht zum Einsatz der Videografie in der Psychiatrie und Psychotherapie. Die theoretische und empirische Basis von Furchtappellen als Mittel zur Einstellungs- und Verhaltensänderung sollen beschrieben und ausgehend von den benannten Grundlagen die klinischen Hypothesen zur Abbruchquote abgeleitet werden. Diese werden den Fokus der folgenden empirischen Überprüfung darstellen.

\subsubsection{Epidemiologische und gesundheitsökonomische As- pekte der Suchterkrankungen}

Im Folgenden sind einleitend zunächst Daten zur Häufigkeit der Suchterkrankungen in der Bundesrepublik Deutschland aufgeführt:

Dem Drogen- und Suchtbericht der Bundesregierung vom Mai 2011 (Drogenbeauftragte der Bundesregierung, 2011) zufolge konsumieren nach aktuellen Schätzungen in Deutschland 200.000 Menschen illegale Drogen in riskanter Form, wobei Cannabiskonsumenten ausgeschlossen wurden. Die Mehrheit der Betroffenen injiziert Heroin.

600.000 Menschen betreiben derzeit in Deutschland einen missbräuchlichen oder abhängigen Cannabiskonsum. 
Bei 1,3 Millionen Menschen besteht eine Alkoholabhängigkeit, bei weiteren 9,5 Millionen liegt ein riskanter Alkoholkonsum vor.

1,4-1,9 Millionen Personen sind medikamentenabhängig, davon 70\% Frauen.

Nicht zuletzt aufgrund einer deutlich erhöhten Komorbidität Drogenabhängiger mit HI- und Hepatitis-C-Virusinfektionen stellen die Suchterkrankungen ein gravierendes epidemiologisches und gesundheitsökonomisches Problem dar: Heroinabhängige weisen im Vergleich zu gesunden Kontrollpersonen eine 13-mal erhöhte Mortalität auf (Hulse et al. 1999), bei injizierenden Heroinabhängigen sogar noch höher (Sporer 2003). Pro Jahr liegt die Mortalität bei der Opioidabhängigkeit zwischen 1 und 3\% (Bühringer et al. 1997; Segest et al. 1990; Staak und Rupp 1993). Dabei betrifft die Gefahr des Todes entsprechend der Altersstruktur Opioidabhängiger vor allem jüngere Menschen - also eine Altersgruppe mit einem generell eher niedrigen Mortalitätsrisiko. Im Drogen- und Suchtbericht 2012 wird der Altersdurchschnitt der Drogentoten mit 37Jahren angegeben (Drogenbeauftragte der Bundesregierung, 2012).

Unter anderem im Hinblick auf die Mortalität stellen Abbrüche stationärer Entgiftungsbehandlungen durch die Patienten kritische Ereignisse dar (Toleranzverlust).

\subsubsection{Behandlungsabbrüche im stationären Drogenentzug sowie Behandlungsstrategien}

Die Behandlung drogenabhängiger Patienten ist komplex und schwierig: Behandlungsabbrüche sind dabei häufig und stellen ein kritisches Ereignis besonders auf Drogenentzugsstationen dar. Dies nicht nur, weil sie frustrierend und demotivierend gleichermaßen auf Mitpatienten wie auch auf das Behandlungsteam wirken, sondern auch, weil - wie Rössler et al. (1993) berichten - nach vorzeitigem Behandlungsende das Risiko für potentiell letale Überdosierungen deutlich ansteigt. Außerdem gibt es Angaben darüber, dass sich bei einem Teil methadonsubstituierter Patienten nach belastenden Entgiftungserfahrungen Symptome einer Entgiftungsphobie entwickeln, welche hinsichtlich der emotionalen Reaktion einer Posttraumatischen Belastungsstörung gleichen (Milby et al. 1994). 
Um die Entzugssymptomatik zu dämpfen und damit die Haltequote zu verbessern bzw. die Abbruchquote in der Entgiftungsbehandlung zu verringern, werden vielfältige therapeutische Mittel eingesetzt - darunter u.a. die medikamentöse Behandlung mit Clonidin (Schanda et al. 1983), die Verordnung von Antidepressiva (McCaul et al. 1984), von Neuroleptika (Gastpar und Rösinger 1996) sowie der Einsatz von Codein (Banbery et al. 2000). Wie häufig sind dennoch Abbrüche der Entgiftungsbehandlung zu verzeichnen?

Trotz der sich seit Ende der 80er Jahre immer mehr durchsetzenden Methadongestützten Opioidentgiftung und der Hoffnung, mit einem sogenannten „warmen Entzug" die Haltequote verbessern zu können, zeigen verschiedenste Studien Durchhalteraten kaum oberhalb von $\mathbf{5 0 \%}$ (siehe Tabelle 1, S. 4: Übersicht zu Abbruchquoten bei stationären Entgiftungen).

\section{Tabelle 1: Abbruchquoten bei stationären Entgiftungen} (modifiziert nach Gößling et al. 2001, S. 475)

\begin{tabular}{|c|c|c|c|c|c|c|}
\hline & $\begin{array}{l}\text { Hammer } \\
\text { Modell } \\
\text { Raschke } \\
\text { et al. } \\
(1985)\end{array}$ & $\begin{array}{l}\text { Hamburg } \\
\text { Ochsenzoll } \\
\text { Trüg (1992) }\end{array}$ & $\begin{array}{l}\text { Königslutter } \\
\text { Esse et al. } \\
\text { (1994) }\end{array}$ & $\begin{array}{l}\text { Osnabrück } \\
\text { Schulz et } \\
\text { al. (1995) }\end{array}$ & $\begin{array}{l}\text { Lüneburg } \\
\text { Wild et } \\
\text { al. (1995) }\end{array}$ & $\begin{array}{l}\text { Universität } \\
\text { Bonn } \\
\text { Neumann } \\
\text { et al. } \\
\text { (2002) }\end{array}$ \\
\hline Untersuchungszeitraum & $1970-82$ & $1989 / 90$ & 1993 & $1993 / 94$ & $1993 / 94$ & $1990-94$ \\
\hline Stichprobengröße & 135 Pat. & 201 Pat. & 262 Pat. & 175 Pat. & 217 Pat. & 179 Pat. \\
\hline Zugangsweg & $\begin{array}{c}\text { hoch- } \\
\text { schwellig }\end{array}$ & $\begin{array}{l}\text { hochschwellig } \\
\text { und nieder- } \\
\text { schwellig }\end{array}$ & $\begin{array}{l}\text { nieder- } \\
\text { schwellig }\end{array}$ & $\begin{array}{l}\text { nieder- } \\
\text { schwellig }\end{array}$ & $\begin{array}{l}\text { nieder- } \\
\text { schwellig }\end{array}$ & $\begin{array}{l}\text { nieder- } \\
\text { schwellig }\end{array}$ \\
\hline $\begin{array}{l}\text { Durchschnittsalter bei } \\
\text { Aufnahme in Jahren }\end{array}$ & 19,2 & 26,2 & 25,9 & 26,6 & 27,4 & 28,6 \\
\hline \multicolumn{7}{|l|}{ Geschlechterverteilung } \\
\hline Männer & $58,0 \%$ & $66,0 \%$ & $67,9 \%$ & $78,3 \%$ & $69,9 \%$ & $53,6 \%$ \\
\hline Frauen & $42,0 \%$ & $34,0 \%$ & $32,1 \%$ & $21,7 \%$ & $34,1 \%$ & $46,4 \%$ \\
\hline Behandlungsdauer & 7 Monate & $?$ & 14,0 Tage & 21,2 Tage & $\begin{array}{r}14,6 \\
\text { Tage }\end{array}$ & $?$ \\
\hline Abbruchquote & $54,1 \%$ & $53 \%$ & $57,1 \%$ & $47,2 \%$ & $52,4 \%$ & $34,6 \%$ \\
\hline
\end{tabular}


Entgegen optimistischen Publikationen aus dem angloamerikanischen Bereich aus den 80er Jahren (Craig et al. 1982; Gossop et al. 1989) konnten mittels Verabreichung von Methadon lediglich die Frühabbruchraten in den ersten Behandlungstagen gesenkt werden (s.u.), jedoch kommt es nicht zu einer Reduktion der Gesamtabbruchrate (Gößling et al. 1998). Verstärkte Aufmerksamkeit sollte also der Frage zukommen, wie das sich beim Methadon-gestützten Entzug eröffnende therapeutische Fenster effektiv genutzt werden kann.

Aufgrund der unbefriedigenden Erfolgsquoten kam schließlich auch dem sogenannten „Turboentzug“ unter Vollnarkose verstärkt Beachtung zu (Loimer et al. 1991; Bartter und Goobermann 1996; Brewer 1996). Für diese durch Opiatantagonisten induzierten Entzugsbehandlungen in Narkose kommt nach bisherigen Erfahrungen jedoch nur eine hochselektierte Klientel in Frage (Gößling et al. 1997). Auch bei diesen Patienten manifestieren sich im weiteren Verlauf nach dem Eingriff Beschwerden, die mit denen einer konventionellen Entgiftung vergleichbar sind (Scherbaum et al. 1998, Boehle et al. 2000). Außerdem liegen Berichte über vital bedrohliche Komplikationen vor: So beschrieben Pfab et al. (1996) einen Fall von Lungenversagen und zwei Fälle von kompensiertem Nierenversagen. Brewer (1997) nennt in seiner Literaturübersicht bis 1997 vier Todesfälle in engem zeitlichem Zusammenhang zum „Turboentzug“.

Bei einer 20-tägigen Methadon-gestützten Entgiftung kommt es seltener zu Frühabbrüchen, die meisten Behandlungsabbrüche ereignen sich ab der 3 . Behandlungswoche. Darauf wies Cook schon 1989 hin und brachte dieses Phänomen damit in Zusammenhang, dass die Entzugssymptome am Ende der Entgiftung am stärksten sind.

In dieser Phase gefährdet eine große Rückfallneigung den Erfolg der Entzugsbehandlung. Es wurde auch versucht, auf medikamentösem Wege die Entzugssymptomatik und das Verlangen nach der Droge zu unterdrücken (Gallimberti et al. 1993; McCaul et al. 1984), jedoch bisher ohne nachhaltigen Erfolg. Therapieabbrüche stellen so weiterhin ein kritisches Ereignis auf Drogenentzugsstationen dar: Bei Therapieabbrechern wurde ein signifikant stärkerer Anstieg dysphorischer Verstimmungen beobachtet. Insbesondere bei der Entgiftung von langzeitsubstituierten Methadon-Patienten wurde ein organisch determiniertes dysphorisches 
Syndrom postuliert, welches die Entwicklung eines negativ gefärbten Selbsterlebens sowie eine anhaltend pessimistische Zukunftserwartung begünstigt (Kanof et al. 1993; Latowsky 1996).

In neueren Arbeiten wird über den Einsatz von Buprenorphin bei der Opioidentgiftung berichtet. Paetzold et al. (2000) beschreiben, dass darunter weniger depressive Verstimmungen im Gesamtverlauf der Entgiftung auftreten als unter der Gabe von d-I-Methadon bzw. L-Polamidon (Brack 2002). Bickel et al. (1988) berichteten darüber hinaus über eine bessere Haltequote beim Entzug mit Buprenorphin gegenüber der methadongestützten Entgiftung, während Brack (2002) diesbezüglich keine Unterschiede feststellte. Nach heutigem Wissensstand sind Methadon und Buprenorphin ähnlich effektiv in der Entgiftungsbehandlung sowie auch der Substitutionstherapie opioidabhängiger Patienten, wobei Buprenorphinnaloxon ein geringeres Missbrauchspotential besitzt (Boothby und Doering 2007). Brack und Behrend (2004) fanden in Entzugsbehandlungen mit Methadon bzw. Buprenorphin keinen Unterschied in der Haltequote, wenngleich die Entzugssymptome unter Buprenorphin in den letzten Tagen der medikamentösen Behandlung geringer ausgeprägt waren.

Neben den genannten medikamentösen Behandlungsstrategien kommen adjuvant unterschiedliche weitere Therapieverfahren im Rahmen der Entgiftungsbehandlung zum Einsatz:

- Die Akupunktur (Brewington et al. 1994; Moner 1996) wird inzwischen seit über 20 Jahren als Entzugsmethode sowie als Mittel zur Rückfallprophylaxe eingesetzt. Hinsichtlich des Forschungsstandes zu ihrer Wirksamkeit bietet sich ein uneinheitliches Bild. Zwar zeigen die vorliegenden kontrollierten Studien die prinzipielle Effektivität der Akupunktur zur Linderung von Entzugs- und Cravingsymptomen beim Opiatentzug, die erzielten Effekte sind jedoch insgesamt relativ gering. Darüber hinaus ist die Aussagekraft der Studien durch hohe Drop-out-Raten (um 80\%) eingeschränkt.

- Außerdem liegen Veröffentlichungen über verschiedene psychotherapeutische Verfahren zur Suchtbehandlung vor, meist jedoch ohne Berücksichtigung ihres Einsatzes im Rahmen einer Entgiftungsbehandlung (Platt et al. 1990). Darunter befinden sich verhaltenstherapeutische Methoden wie sys- 
tematische Desensibilisierung und Entspannungstraining (O'Brien JS et al. 1972), kognitiv verhaltenstherapeutische Techniken und Kontingenzmanagement (Carroll 2005) sowie EMDR (Shapiro et al. 1994). Die Motivierende Gesprächsführung (Demmel 2001; Miller und Rollnick 1999) kam bisher hauptsächlich bei der Behandlung der Alkoholkrankheit zum Einsatz, obwohl sie inzwischen zunehmend auch zur Anwendung bei der Behandlung von Drogenabhängigen kommt. Die Dialektisch-Behaviorale Therapie in der von Linehan (1996 a, 1996 b) entwickelten Form eignet sich vor allen Dingen zur Behandlung einer zusätzlich bestehenden Borderline-Störung (Stuppe 2002). Bei Komorbidität mit einer Suchterkrankung zeigten sich die bekannten Effekte auf die Borderlinepathologie in gleicher Weise, wohingegen keine signifikante Beeinflussung der Suchterkrankung nachweisbar war (van den Bosch et al. 2002). Neuerdings ist auch eine Weiterentwicklung der Dialektisch-Behavioralen Therapie unter Einbeziehung suchtspezifischer Aspekte erschienen (Lüdecke et al. 2010).

Bisher liegen jedoch keine Untersuchungen über den spezifischen Einfluss dieser Therapien auf die Haltequote im Rahmen stationärer Drogenentgiftungsbehandlungen vor.

\subsubsection{Einflussfaktoren auf Behandlungsabbrüche im statio- nären Drogenentzug}

Es existiert eine ganze Reihe von Studien zu Einfluss nehmenden Faktoren bezüglich Behandlungsabbrüchen in stationären Entgiftungsbehandlungen drogenabhängiger Patienten. Diese kommen zu sehr unterschiedlichen Ergebnissen Die Vielzahl an potentiellen Wirkfaktoren sowie die zum Teil ausgeprägte Uneinheitlichkeit der Befundlage sollen im Folgenden zum Ausdruck gebracht werden:

Nach einer Untersuchung von Gößling et al. (2001) zu Bedingungsfaktoren im stationären Drogenentzug wird das therapeutische Stationsprogramm von Therapieabbrechern kritischer beurteilt als von Durchhaltepatienten. Untersuchungen des therapeutischen Settings als Einflussgröße auf den Behandlungserfolg deuten darauf hin, dass auf spezialisierten Entgiftungsstationen signifikant höhere Raten 
erfolgreich abgeschlossener Entgiftungen erzielt werden als auf allgemeinpsychiatrischen Stationen (Strang et al. 1997). Dem gegenüber brachte eine vom Patienten selbst bestimmte Dosierung des Methadons keine besseren oder schlechteren Ergebnisse als die ärztlich bestimmte Dosierung (Senay et al. 1984).

In der Untersuchung von Gößling et al. (2001) war die Abbruchquote von Frauen im stationären Drogenentzug signifikant höher gegenüber derjenigen bei den Männern (68,4\% vs. 44,2\%), die Therapieabbrecher waren im Durchschnitt 2,2 Jahre jünger als Patienten mit regulärem Therapieabschluss. Der strafrechtliche Status hatte keinen Einfluss auf das Durchhaltevermögen, während eine länger dauernde Abstinenzphase in der Vorgeschichte die Chance auf ein reguläres Behandlungsende erhöhte. Auch das Ziel einer anschließenden Entwöhnungsbehandlung korrelierte positiv mit einer höheren Durchhalterate. Hervorgehoben wurde, dass Patienten, die ihre Behandlung vorzeitig abbrechen, ihr seelisches Befinden und ihren körperlichen Zustand deutlich pessimistischer einschätzen als Patienten mit regulärem Behandlungsabschluss.

Rüesch und Hättenschwiler (2002) betonen, dass Drogenrückfälle signifikant mit Behandlungsabbrüchen im stationären Drogenentzug korrelieren. Sie hinterfragen den restriktiven Umgang mit Drogenrückfällen kritisch, da bekannt ist, dass die Art der Entzugsbeendigung als wichtigster Prädiktor für den langfristigen Therapieerfolg gilt (Roch et al. 1992).

Hirsch und Tretter (2000) wiesen nach, dass Therapieabbrecher im Vergleich zu regulären Therapiebeendern häufiger mit einer Bezugsperson zusammen lebten, eine eigene Wohnung und bereits Vorbehandlungen sowie eine kurze Wartezeit hatten. Günstig für eine reguläre Entlassung war eine länger währende vorherige Methadonsubstitution. In einer Untersuchung von Backmund et al. (2001) hingegen stand die Wartezeit auf den Therapieplatz in keinem Zusammenhang zum erfolgreichen Abschluss einer Entgiftungsbehandlung. Übereinstimmend mit Hirsch und Tretter (2000) kommen auch Backmund et al. (2001) zu dem Ergebnis, dass substituierte Patienten eine Entgiftungsbehandlung eher erfolgreich beenden als Heroinabhängige (50,4\% der Methadonsubstituierten, 45,5\% der Codeinsubstituierten, 35,9\% der Heroinabhängigen beendeten Entgiftungsprogramm erfolgreich). Die Autoren vermuten, dass Methadonprogramme (Grönbladh et al. 1990; 
Backmund et al. 2001; Marsch 1998) und Codeinprogramme (Krausz et al. 1998) die Patienten stabilisieren, wobei Patienten in einem Methadonprogramm im Vorfeld einer Entgiftungsbehandlung die beste psychosoziale Unterstützung erleben. Des Weiteren fanden Backmund et al. (2001) folgende Prädiktoren für eine erfolgreiche Entgiftungsbehandlung: Steigendes Alter (bis 28 Jahre), bessere Ausbildung, regelmäßiger Kontakt zu einem Berater, Vorliegen einer Bewährung sowie Pläne an einer Entwöhnungsbehandlung teilzunehmen. Die Anzahl der Vorstrafen korrelierte negativ mit dem Entgiftungserfolg, wobei dieser Faktor erst unter Hinzuziehung des Alters signifikant wurde. Mehr psychosoziale und psychotherapeutische Unterstützung führte zu einem besseren Ergebnis der Entgiftungsbehandlung.

Hirsch und Tretter (2000) weisen darauf hin, dass eine gute soziale und forensische Situation als Desmotivator wirken kann. Sie unterscheiden interne und externe Faktoren der Therapiemotivation und kommen zu dem Schluss, dass die Haltekraft der Entzugstherapieeinrichtungen zu einem hohen Anteil von einer Vielzahl nur geringgradiger Wirkgrößen bestimmt zu sein scheint, die sich nach Meinung der Autoren zu einem großen Teil der Reichweite therapeutischen Handelns entziehen.

Hinsichtlich der Suche nach Prädiktoren für das Durchhaltevermögen berichten Neumann et al. (2002) in ihrer Untersuchung einer niederschwelligen qualifizierten Drogenentzugsbehandlung darüber, dass sich günstig hinsichtlich der Abbruchwahrscheinlichkeit eine positive Familienanamnese für eine depressive Störung und die Anzahl der bereits durchgeführten Langzeittherapien erwiesen. Alle anderen dort untersuchten Parameter (soziodemographische Variablen wie Alter, Geschlecht, forensische Anamnese, Prostitution, Arbeitslosigkeit, Konsummuster in der Vergangenheit, Konsummuster vor der stationären Therapie) zeigten in dieser Untersuchung keinen signifikanten Einfluss auf das Abbruchverhalten.

Petry und Bickel (1999) beforschten Einflüsse der Qualität der therapeutischen Beziehung und des psychiatrischen Störungsschweregrades auf die Therapiebeendigung bei Opiatabhängigen. Die Autoren stellten fest, dass Therapieabbrecher ein schweres Störungsbild aufweisen. In dieser Subgruppe wirkt sich die Qualität der therapeutischen Beziehung signifikant auf die Art der Therapiebeendigung 
aus: Eine gute therapeutische Beziehung führt bei Patienten mit einem schweren Störungsbild zu einer dreifach erhöhten regulären Therapiebeendigung gegenüber Patienten, die die therapeutischen Beziehung weniger gut bewerten. Die Therapiebeender sind durch einen leichten bis mittleren Schweregrad ihrer Symptomatik gekennzeichnet und der Einfluss der therapeutischen Beziehung ist bei ihnen nicht relevant.

In Untersuchungen von San et al. (1989) und Armenian et al. (1999) standen Patientenvariablen wie Geschlecht, Alter, Dauer des Drogengebrauchs, Ausbildungssowie Beschäftigungsverhältnis nicht in Zusammenhang mit dem Behandlungsergebnis. In anderen Studien wurde festgestellt, dass Patienten, die die Behandlung gegen ärztlichen Rat beendeten, jünger waren, keinen Hausarzt hatten, eine Vorgeschichte mit Behandlungsabbrüchen aufwiesen (Jeremiah et al. 1995), öfter Single waren, vor Aufnahme auf die Entgiftungsstation Kokain konsumiert hatten (De los Cobos et al. 1997; Armenian et al. 1999) und einen schwereren Drogengebrauch sowie schwerere medizinische Probleme nach dem Addiction Severity Index hatten (Franken und Hendriks 1999). Psychopathologie und soziodemografische Variablen sagten das Ergebnis der Detoxifikation in einer Studie von Franken und Hendriks (1999) nicht voraus.

Den Ergebnissen von Backmund et al. (2001) zufolge stand der erfolgreiche Abschluss einer Entgiftungsbehandlung weder in Zusammenhang mit einem täglichen Konsum anderer Drogen noch mit dem Alter bei Beginn der Sucht.

Küfner (1995) beleuchtet diverse Behandlungsfaktoren im Rahmen der Drogenentwöhnungsbehandlung. Dabei stellt er u.a. fest, dass niedrige Abbruchquoten zugleich in Zusammenhang stehen mit höheren Rückfallquoten. Eine liberalere Haltung der Einrichtung war dabei mit höheren Haltequoten, aber auch erhöhtem Rückfallrisiko in der Behandlung verbunden. Er plädiert aufgrund therapeutischer Überlegungen eher für die größere Wichtigkeit der Haltequote, da nur beim Verbleib der Patienten eine therapeutische Aufarbeitung der Bedingungen des Rückfalls möglich ist. Diese wird allerdings durch den Suchtdruck auf der anderen Seite erheblich erschwert. Komplexe Verleugnungsstrategien (Subkowski 2000) seitens der Patienten bezüglich ihrer Behandlungsbedürftigkeit kommen weiterhin erschwerend hinzu. 
Insgesamt wurde im Rahmen dieser Ausführungen verdeutlicht, wie uneinheitlich und vielschichtig die Befundlage zu personenspezifischen und behandlungsspezifischen Einflüssen auf das Durchhaltevermögen der suchtkranken Patienten ist. Es wird wichtig sein, weiteres Wissen darüber zu gewinnen, welche Patienten von welchen möglichen Behandlungsangeboten bzw. -strategien profitieren können. Nur so ließe sich die Nutzung von Ressourcen im Rahmen der stationären Entgiftungsbehandlung weiter optimieren. Es gilt herauszufinden, welche Patienten ein an die speziellen Bedürfnisse dieser Patientengruppe angepasstes Therapieangebot benötigen und wie dieses Angebot beschaffen sein müsste. Ziel ist es, den größtmöglichen Behandlungserfolg zu erzielen und die Haltequoten weiter zu verbessern. Die vorliegende Arbeit versucht, zu diesen Bemühungen ebenfalls einen Beitrag zu leisten. Berücksichtigung finden muss dabei u.a. auch das Komorbiditätsproblem.

\subsection{Die Problematik der Diagnostik komorbider psychiatri- scher Störungen im Rahmen des stationären Entzugs illegaler Drogen}

Die Komorbidität psychiatrischer Störungen bei drogenabhängigen Patienten ist erheblich. Diese spezielle Form der Komorbidität (Kombination von substanzbezogenen Störungen und psychischen Störungen ohne Substanzbezug) wird als ein immer wichtiger werdendes Problem psychiatrischer Klassifikation (Maser und Patterson 2002) und Therapie beurteilt (O’Brien CP et al. 2004). Das Konzept der substanzinduzierten Störungen versus -unabhängigen Störungen wird zudem inzwischen als eine Erweiterung des „Primär/sekundär“-Ansatzes angesehen (Grant et al. 2004).

Während einer Entgiftungsbehandlung ist die valide Diagnostik komorbider psychiatrischer Störungen allerdings nahezu unmöglich: Dem zugrunde liegt der Umstand, dass zu Beginn einer Entgiftungsbehandlung häufig Intoxikationen bzw. Mischintoxikationen oder auch Entzugssyndrome bestehen. Letztere prägen auch den psychopathologischen Zustand der Probanden in der Endphase der Entgif- 
tungsbehandlung. Insgesamt resultiert aufgrund der Überlappung der Syndrome zum einen sowie der Kürze der Behandlungsdauer einer 3- bis 4-wöchigen stationären Entgiftung zum anderen ein erhebliches Risiko an Fehldiagnosen: Es ist bekannt, dass manche Substanzen z.B. Alkohol oder Sedativa bei Intoxikationen zeitweise Zustände verursachen können (z.B. Depressionen oder Angstzustände), die bestimmten psychischen Störungen ohne Drogen ähneln. Andere Drogen (z.B. Psychostimulanzien) wiederum können diese Zustände im Entzug auslösen, während sie bei Intoxikationen z.B. maniforme oder psychotische Zustände verursachen. Diese sind von den entsprechenden psychiatrischen Störungen an sich nur schwer und in manchen Fällen erst im Rahmen einer langfristigen Verlaufsbeobachtung zu unterscheiden.

Raskin und Miller (1993) weisen darauf hin, dass psychiatrische Symptome, die in Zeiten aktiven Substanzkonsums auftreten, unter einer Behandlung innerhalb von Wochen bis Monaten zurück gehen können. Die Autoren betonen, dass die Beurteilung einer vom Substanzgebrauch unabhängigen psychischen Störung Beobachtungszeiträume zwischen 4 Wochen und 2 Jahren voraussetzt und auch von der Art der Diagnosen abhängt.

Die Zusatzdiagnose einer Persönlichkeitsstörung weist außerdem eine Streubreite mit Prävalenzraten von 7-100\% (Clerici und Carta 1996; Kuntze et al. 1998) auf. Selbst bei ein und derselben Stichprobe können je nach Messverfahren unterschiedliche Einschätzungen resultieren (Marlowe et al. 1997), zumal die Messinstrumente nicht immer an Suchtkranken validiert sind.

Einen weiteren möglichen Erklärungsansatz für die unterschiedlichen Prävalenzraten im Verlauf von Suchterkrankungen liefern Kipp und Stolzenburg (2000): Die Autoren gehen davon aus, dass sich im Rahmen einer Suchtentwicklung das gesamte Funktionsniveau der süchtigen Person zunehmend regressiv wandelt und zwar auf eine frühe Funktionsstruktur.

Außerdem sind die Populationen klinischer Studien nicht identisch mit denen epidemiologischer Studien. Klinische Studien liefern aus epidemiologischer Sicht oft zu hohe Prävalenzraten, da der Substanzgebrauch häufig mit einer Exazerbation der komorbiden psychischen Störung einhergeht. 
Werden die genannten Aspekte berücksichtigt, so ist es nicht verwunderlich, dass die Prävalenzraten psychiatrischer Störungen bei Suchterkrankungen erheblich im Rahmen unterschiedlicher Behandlungssettings variieren: Gossop et al. (2006) beschreiben, dass sich psychiatrische Symptome bei Drogenabhängigen nach Beginn einer ambulanten oder stationären Behandlung der Suchterkrankung verringern. Diese Remissionen ereigneten sich recht schnell - im ersten Monat - und blieben bis zur Nachuntersuchung (nach einem Monat und sechs Monaten) erhalten: Zu Beginn hatten 39\% der stationären Patienten Zeichen einer komorbiden psychiatrischen Erkrankung, während es sowohl nach einem Monat als auch nach sechs Monaten nur noch 3\% waren. Bei den Methadon-Patienten erfültten 15\% initial die Kriterien einer weiteren psychiatrischen Erkrankung, und 5\% nach einem Monat bzw. sechs Monaten. Diejenigen Personen, die in Behandlung blieben, zeigten eher Verbesserungen hinsichtlich der psychiatrischen Symptome. Die Anzahl der Patienten, die über Suizidideen berichteten, verringerte sich drastisch und hatte sich an beiden Nachuntersuchungspunkten mehr als halbiert.

Bei einer großen australischen Studie zur Therapie Heroinabhängiger, wurde festgestellt, dass die Rate an depressiven Erkrankungen im Verlauf der Behandlung der Suchterkrankung nach 12 Monaten von 26\% auf 11\% gesunken war (Havard et al. 2006).

Raskin und Miller (1993) wiesen darauf hin, dass die Prävalenzrate depressiver Störungen bei Suchtkranken in einer psychiatrischen Umgebung $30 \%$ betrug, während sie in einer anderen Umgebung des Suchthilfesystems bei $5 \%$ lag. Bei den Angststörungen ließen sich ähnliche Befunde nachweisen (30\% versus 3\%). Trotz deutlicher Streuung der Prävalenzraten (besonders hoch in klinischer Umgebung) tritt Komorbidität in unterschiedlichen Populationen oft in ähnlicher Weise auf, z.B. in unterschiedlichen Ländern, bei abstinenzorientierten Behandlungsansätzen ebenso wie bei substitutionsgestützten Behandlungen (Günther et al. 2000).

Psychiatrischen Zusatzdiagnosen, die im Rahmen einer Entgiftungsbehandlung gestellt werden, kommt meist wenig Gültigkeit zu. Dennoch sollen bei der Interpretation der Ergebnisse der vorliegenden Studie Komorbiditäten nicht gänzlich unberücksichtigt bleiben. In einem nächsten Kapitel wird daher ein Überblick über die 
empirischen Befunde zur psychiatrischen Komorbidität und deren Einfluss auf die Behandlung drogenabhängiger Personen gegeben. Anschließend soll auch auf geschlechtsspezifische Besonderheiten eingegangen werden.

\subsubsection{Empirische Befunde zur Häufigkeit komorbider psy- chiatrischer Störungen und zur Suizidalität bei Dro- genabhängigkeit}

Die Komorbidität psychiatrischer Störungen ist nicht etwa nur ein Phänomen, das besonders bei Drogenabhängigen zu beobachten ist, sondern stellt bei der Betrachtung der Lebenszeitprävalenzen psychischer Störungen eher die Regel dar (Kessler et al. 1994; Kessler et al. 1996; Wittchen et al. 1992).

Es wurde aufgezeigt, dass unter Drogenabhängigen psychische Störungen nach ICD-10 weitaus häufiger auftreten als in der sogenannten Normalbevölkerung.

\section{Befunde zur allgemeinen Komorbidität psychiatrischer Störungen}

Bei Opiatabhängigen wurden in großen epidemiologischen Studien wie der „Epidemiologic Catchment Area (ECA) Study" eine Lebenszeitprävalenz komorbider psychischer Störungen von 65\% nachgewiesen (Regier et al. 1990). Damit ist die Rate an psychischen Störungen 2-3-mal höher als in der Allgemeinbevölkerung. Andere ausschließlich an Opiatabhängigen durchgeführte Studien fanden sogar Prävalenzraten von bis zu 90\% (Basdekis-Jozsa und Krausz 2002)

Nach einer Untersuchung von Krausz et al. (1998) liegt die Lebenszeitprävalenz für mindestens eine weitere psychische Störung bei Opiatabhängigen bei $62 \%$. Lässt man die Persönlichkeitsstörungen unberücksichtigt, dann reduziert sich die Lebenszeitprävalenz auf $55 \%$. Es wurden bei $43 \%$ der Opiatabhängigen nach ICD-10 Störungen der Kategorie F4 diagnostiziert (neurotische, Belastungs- und somatoforme Störungen), bei insgesamt 32\% affektive Störungen (F3) und bei 5\% der Untersuchten wurde eine Psychose aus dem schizophrenen Formenkreis (F2) diagnostiziert.

Ähnliche Zahlen geben Günther et al. (2000) zur Komorbidität bei Drogenabhängigen in einer Entgiftungsbehandlung an. Hier lag die Lebenszeitprävalenz komorbi- 
der psychiatrischer Störungen ebenfalls bei 55\%, wobei Angst- und Zwangsstörungen mit 50\% und affektive Störungen mit 38\% dominierten. In einer Entwöhnungsbehandlung gaben $82 \%$ an, in ihrem Leben schon einmal unter ernsten psychischen Problemen gelitten zu haben.

Bei erwachsenen Drogenabhängigen wurde in 11-35\% die Zusatzdiagnose eines Aufmerksamkeit-Defizit-Hyperaktivitätssyndroms gestellt, wohingegen es in der Allgemeinbevölkerung unter 1-5\% der Erwachsenen auftritt (Kalbag und Levin 2005).

\section{Befunde zur Suizidalität}

Auch suizidale Verhaltensweisen sind häufig bei Abhängigkeitserkrankungen gegeben. Preuss et al. (2004) beziffern dabei den Anteil vollendeter Suizide auf mindestens 25-33\% und Suizidversuche sind sogar um das 10-20-Fache häufiger (Diekstra und Gulbinat 1993). In einer Übersicht über Suizidalität bei verschiedenen psychiatrischen Störungen wurde angegeben, dass das Mortalitätsrisiko für einen vollendeten Suizid bei Heroinabhängigen um das 14-Fache erhöht ist (Harris und Barraclough 1997). Auch andere Autoren bestätigen ein deutlich erhöhtes Risiko für vollendete Suizide bei dieser Personengruppe (Übersicht bei Darke und Ross 2002).

Die Risiken für Suizidalität steigen gegenüber Störungen durch Einzelsubstanzen nochmals, wenn ein kombinierter Substanzmittelkonsum vorliegt. So geben Harris und Barraclough (1997) ein insgesamt 3,4-fach erhöhtes Risiko für einen vorzeitigen Todesfall bei dieser Personengruppe an. Vollendete Suizide werden 39-mal häufiger als in der Vergleichsgruppe begangen. In der NCS-Studie wurde der kumulative Einfluss multiplen Substanzmittelkonsums auf das Risiko für Suizidversuche nachgewiesen. Dieses steigt bei Konsum von zwei Substanzen auf das 4,2Fache, bei Einnahme von vier Substanzen auf das 11,3-Fache und auf das 27,4Fache, wenn sechs oder sieben Substanzen konsumiert werden (Borges et al. 2000).

Im Gegensatz zu den oben genannten Befunden war das Risiko für Suizidversuche bei Heroinabhängigen in der NCS-Studie überraschenderweise nicht erhöht, 
während Personen mit Missbrauch und Gebrauch eine deutlich erhöhte Wahrscheinlichkeit für suizidales Verhalten aufwiesen (Borges et al. 2000).

\section{Prävalenz von Traumatisierungen und komorbiden Posttraumatischen Be- lastungsstörungen bei Drogenabhängigen}

Auch Traumatisierungen und Posttraumatische Belastungsstörungen vorwiegend durch sexuellen Missbrauch und Gewalterfahrungen kommen bei drogenabhängigen Patienten häufiger vor als in der Normalbevölkerung. Nach Finkelhor (1994) ist davon auszugehen, dass 10-15\% der Frauen und 5-10\% der Männer der Normalbevölkerung in Europa und den Vereinigten Staaten bis zum Alter von 16 Jahren mindestens einmal zu einem „sexuellen Körperkontakt“ gezwungen werden. Die Quote von Missbrauch überhaupt (auch ohne „sexuellen Körperkontakt“) wird auf bis zu 25\% geschätzt. Missbrauch zum Geschlechtsverkehr betrifft 2-2,3\% aller Mädchen und 1-1,5\% aller Jungen.

Kessler et al. (1995) fanden eine Lebenszeitprävalenz für die PTBS von 7,8\%, dabei waren Frauen mehr als doppelt so häufig betroffen wie Männer (10,4\% vs. $5,0 \%)$.

Kreyssig (1997) beziffert den Anteil sexuell missbrauchter Frauen an allen drogensüchtigen Frauen in Deutschland auf 70\%.

Nach den Ergebnissen einer neueren deutschen Multi Center Studie haben 34,1\% der Behandlung suchenden Personen mit kombinierter Alkohol- und Drogenabhängigkeit eine posttraumatischen Belastungsstörung, wobei mehr Frauen als Männer betroffen sind (Driessen et al. 2008).

Krausz und Briken (2002) fanden bei opiatabhängigen Frauen einen Anteil von 41,3\% mit sexuellen Missbrauchserfahrungen, die häufig mit physischer Misshandlung und emotionalem Missbrauch assoziiert sind. Nach unseren klinischen Erfahrungen sind die Symptome einer PTBS bei Opiatabhängigen bzw. Patienten mit polyvalentem Substanzkonsum inklusive einer Opioidabhängigkeit häufig durch die Suchterkrankung kupiert. Es besteht die Gefahr, dass sie im Rahmen einer Entgiftungsbehandlung exazerbieren.

Das Risiko für die Entwicklung einer Substanzabhängigkeit ist nach den Ergebnissen der Epidemiologic-Catchment-Area-Studie (Burnam et al. 1988) bei Frauen 
nach sexuellem Missbrauch erhöht. McCauley et al. (1997) berichten bei kindlicher Traumatisierung über eine 4,7-fach erhöhte Prävalenz von Drogenmissbrauch, eine 2,2-fach höhere Prävalenz von Alkoholabusus, 3,7-fach mehr Suizidversuche und ein 3,2-fach erhöhtes Risiko psychiatrischer Hospitalisierungen.

Traumatisierende Kindheitserfahrungen erhöhen das Risiko für einen Suizidversuch um das 2-5-Fache (Dube et al. 2001).

Außerdem stellt kindlicher Missbrauch einen Risikofaktor für die spätere Entwicklung einer Angststörung oder Depression (Bremner et al. 1993 a, b; Engel et al. 1993; Safren et al. 2002) sowie einer Essstörung (Newton-Taylor et al. 1998) dar. Wöller (2006) beschreibt umfassende Störungen der Emotionsregulierung als ein häufiges Problem in der Behandlung von Patienten mit komplexen Traumafolgestörungen und zeigt verschiedene Behandlungsansätze auf, die sich diesem Problem widmen.

Kilpatrick et al. (1997) beschreiben die Verbindung zwischen Substanzabhängigkeit und traumatisierenden Erfahrungen als Zirkel, in dem das Trauma die Gefahr für die Entwicklung des süchtigen Verhaltens und dieses wiederum die Gefahr erneuter Traumatisierungen erhöht. Auch andere Daten belegen, dass bei einer Suchterkrankung das Risiko für Traumatisierungen und die Entwicklung einer PTBS ansteigt (McCauley et al. 1997). Einen Überblick zu Studien zum Zusammenhang von Traumatisierung und Suchterkrankung bzw. zum Zusammenhang von Traumatisierung, Posttraumatischer Belastungsstörung und Substanzmittelkonsum liefern die Tabellen 26 und 27 im Anhang (S. 117-119).

Die beschriebenen komorbid bestehenden Problembereiche beeinflussen selbstverständlich den Behandlungsverlauf der Betroffenen. Auf diesen Aspekt wird im nun folgenden Abschnitt eingegangen. 


\subsubsection{Empirische Befunde zum Einfluss komorbider psychi- atrischer Störungen auf die Behandlung einer Drogen- abhängigkeit}

Suchtkranke Patienten mit komorbiden psychiatrischen Störungen haben einen erhöhten Substanzkonsum, stärkere soziale Belastungen, und im Verlauf mehr Behandlungsschwierigkeiten gegenüber den Mitpatienten ohne psychiatrische Zusatzdiagnosen.

Das Vorliegen einer komorbiden psychiatrischen Erkrankung bei Drogenabhängigen ist daher im Allgemeinen mit einer schlechteren Behandlungsprognose verbunden (Rounsaville et al. 1986). Gleiches gilt für die Schwere der psychiatrischen Erkrankung (McLellan et al. 1983). Ausschlaggebend für den Behandlungsverlauf scheint die Symptombelastung zu sein. Bei drogenabhängigen Patienten mit einem niedrigen psychiatrischen Symptomlevel ist der Behandlungserfolg im Allgemeinen gut (McLellan et al. 1983; Rounsaville et al. 1986), wohingegen Doppeldiagnosepatienten mit einem höheren Ausmaß psychiatrischer Symptome schlechtere Ergebnisse in den für gewöhnlich suchtfokussierten Behandlungen erzielen.

Eine schnellere Rehospitalisierung wurde bei drogenabhängigen Patienten mit einer drogeninduzierten psychischen Erkrankung gegenüber anderen Suchtpatienten ohne entsprechende Begleiterkrankungen festgestellt (Dixon et al. 1997).

Am Beispiel der depressiven Erkrankungen lassen sich die beschriebenen Gesichtspunkte etwas veranschaulichen:

Auch drogenabhängige Patienten mit komorbiden Depressionen entwickeln sich in einer Suchtbehandlung weniger gut als die Vergleichsgruppe ohne entsprechende komorbide psychiatrische Störung (Ziedonis und Kosten 1991). Im Vergleich zu denen ohne Depressionen, hatten depressive Individuen weniger Kontakt zur Substitutionstherapie und stationären Rehabilitation, verbrachten aber mehr Zeit in der Entgiftung. Sie berichteten über stärkeren Gebrauch von Heroin und anderen Drogen, mehr Risikoverhalten, schlechtere körperliche Gesundheit und mehr psychopathologische Auffälligkeiten. Havard et al. (2006) wiesen bei denjenigen Patienten, die zum Zeitpunkt der Nachuntersuchung (nach 12 Monaten) die Sympto- 
me einer Major Depression aufwiesen, eine insgesamt geringere Zahl an Behandlungstagen bei häufigeren Behandlungsepisoden nach.

Suchtkranke Patienten mit einem Aufmerksamkeitsdefizit-/Hyperaktivitätssyndrom waren im Vergleich zu nicht komorbid erkrankten Süchtigen bei regelmäßigem Konsum jünger, wiesen geringere Intelligenzquotienten auf, litten an spezifischen Persönlichkeitsstörungen und wurden öfter gerichtlich verurteilt (König et al. 2007). Suchtkranke Patienten mit Missbrauchserfahrungen zeigten in einer Studie von Schnieders et al. (2006) sowohl für das körperliche als auch für das psychische Befinden eine deutlich höhere Symptombelastung als die Referenzgruppe ohne sexuelle Missbrauchserfahrungen.

Bei opiatabhängigen Frauen mit sexuellen Missbrauchserfahrungen fanden Krausz und Briken (2002) hinsichtlich der Opiatabhängigkeit keinen problematischeren Verlauf. Allerdings kamen im Zusammenhang mit suizidalen Tendenzen häufiger gewollte Überdosierungen vor. An psychischen Problemen traten aktuell insbesondere Angst- und Spannungssymptome sowie phobische Erkrankungen auf.

In einer Entwöhnungsbehandlung fanden Günther et al. (2000) keinerlei systematischen Einfluss der Komorbidität von Angst- Zwangs- und affektiven Störungen auf einen Behandlungsabbruch der Patienten. Auch nach den Ergebnissen von Roch et al. (1992) korrelieren Klientenmerkmale nur in geringem Ausmaß mit dem Abbruch einer stationären Entwöhnungsbehandlung. Hier könnten Einrichtungsmerkmale relevanter sein (Vollmer 1988; Vollmer und Ellgring 1988).

Dagegen war im Methadonerprobungsvorhaben Nordrhein-Westfalen in der Abbrechergruppe der Anteil der Probanden mit einer oder mehreren psychischen Störungen signifikant höher als in der Gruppe der Nichtabbrecher (89,7 versus 67\%). Möglicherweise schlagen hier die Persönlichkeitsstörungen zu Buche.

Trafton et al. (2006) weisen darauf hin, dass eine Opiat-Substitutionstherapie bei Patienten mit PTBS zwar den Substanzgebrauch reduziert, dass aber zusätzliche therapeutische Maßnahmen nötig sind, um die komorbiden psychiatrischen Probleme anzugehen.

Gleiches gilt auch für suchtkranke Patienten mit anderen psychiatrischen Zusatzdiagnosen. Sie benötigen ein um spezielle Psychotherapieelemente erweitertes 
Behandlungsangebot (Lüdecke et al. 2010). Ein spezialisiertes auf die Mitbehandlung komorbid vorliegender psychiatrischer Störungen zugeschnittenes Therapieangebot verbessert den Behandlungserfolg (McLellan et al. 1983, 1997; Rounsaville und Kleber 1985). Ob ein videobasierter Furchtappell im Rahmen einer stationären Entgiftungsbehandlung eine sinnvolle Erweiterung des Therapieangebots darstellt wurde bisher nicht untersucht und ist Gegenstand der vorliegenden Arbeit.

\subsubsection{Geschlechtsspezifische Besonderheiten drogenab- hängiger Patienten mit komorbiden psychiatrischen Störungen}

In Deutschland sind etwa 30\% der drogenabhängigen Menschen Frauen (Sonntag et al. 2007). Es ist auch bekannt, dass Frauen im Gegensatz zu männlichen Abhängigen signifikant jünger sind, häufiger keinen Schulabschluss haben und häufiger arbeitslos sind. Außerdem steigen sie früher auf die sogenannten harten Drogen um. Zu deren Konsum lassen sie sich oft durch ihre ebenfalls drogenabhängigen Partner verleiten. (Comfort et al. 2003).

Elman et al. (2001) wiesen bei kokainabhängigen Frauen signifikant höhere Craving scores, ein größeres Ausmaß depressiver Symptome und eine höhere Belastung mit sozialen und familiären Problemen nach. Die Autoren vermuten, dass ein Zusammenhang zwischen erhöhtem Craving und weiblichen Geschlechtshormonen besteht, welcher über das dopaminerge System vermittelt wird.

Es ist auch bekannt, dass substanzabhängige Frauen eher dazu neigen, begonnene Therapieversuche abzubrechen (Backmund et al. 2001; Petry und Bickel 2000) bzw., dass sie generell weniger dazu bereit sind, sich einer Therapie ihrer Substanzabhängigkeit zu unterziehen (Green 2006). Andererseits haben Frauen nach einer Behandlung trotz ihrer häufig schwierigeren Lebensumstände eine niedrigere Rückfallrate als männliche Abhängige (Pelissier et al. 2003). 
Darüber hinaus wurden in verschiedenen Untersuchungen weitere Unterschiede zwischen den Geschlechtern bei drogenabhängigen Patienten gefunden, die möglicherweise einen Einfluss auf den Behandlungsverlauf haben und spezielle therapeutische Maßnahmen erfordern, um die Behandlungsergebnisse zu verbessern. Aufgrund der Relevanz des Geschlechts auch in Bezug auf die innere Verarbeitung eines Furchtappells wird nun ein Überblick über die Befundlage erfolgen.

Callaghan et al. (2006) fanden in einer Untersuchung mit kanadischen Aborigenals bei Männern und Frauen am häufigsten Behandlungen wegen Alkoholismus, Frauen hatten jedoch mehr Opiat- und Kokainentgiftungsdiagnosen. Zusätzlich zu einem jüngeren Alter berichteten Frauen über eine größere Rate erlebter körperlicher und sexueller Gewalt. Die Behandlung der Frauen war gekennzeichnet durch eine größere Anzahl medizinischer Untersuchungen und Verordnung von Antidepressiva und Antibiotika.

In einer Studie zur Komorbidität und Sucht in Taiwan berichten Chiang et al. (2007), dass 58,2\% der Männer und 62,5\% der Frauen mindestens eine Achse - I Kodiagnose oder eine antisoziale Persönlichkeitsstörung hatten. Die Frauen waren im Vergleich zu den Männern jünger, schlechter ausgebildet, und hatten einen früheren Beginn der Abhängigkeit illegaler Drogen. Außerdem zeigten sie mehr suizidales Verhalten als Männer. Über eine höhere Suizidalität bei suchtkranken Frauen gegenüber den Männer berichten auch Cottler et al. (2005).

In einer Untersuchung von Günther et al. (2000) wurde bei opiatabhängigen Frauen etwa zweimal so häufig eine weitere psychiatrische Diagnose gestellt im Vergleich zu opiatabhängigen Männern. Insgesamt hatten 57\% der komorbid Opiatabhängigen eine Mehrfachdiagnose. Opiatabhängige Frauen hatten auch häufiger eine zusätzliche dritte psychiatrische Diagnose nach ICD-10 (69\% der Frauen gegenüber $49 \%$ der Männer).

Bei der Lebenszeitprävalenz lagen die Werte der drogenabhängigen Frauen bei den Angst- und Zwangsstörungen sowie den affektiven Störungen mit jeweils 55\% höher als bei den Männern (49\% bzw. 37\%). Auch bei Drogenabhängigen in einer Entwöhnungsgruppe litten mehr Frauen unter Angst- und affektiven Störungen als Männer. 
Es ist bekannt, dass Frauen insgesamt häufiger an einer Posttraumatischen Belastungsstörung erkranken als Männer: Kessler et al (1995) fanden eine Lebenszeitprävalenz für die PTBS von 7,8\%, wobei Frauen mehr als doppelt so häufig betroffen waren als Männer (10,4\% vs. 5,0\%).

Teegen und Zumbeck (2000) untersuchten die Prävalenz traumatischer Erfahrungen und posttraumatischer Belastungsstörungen bei opiatabhängigen Patienten. Auch hier war eine Geschlechterdifferenz feststellbar: Einen sexuellen Missbrauch vor dem 14. Lebensjahr konnten sie bei 38,5\% der Frauen und 4,2\% der Männer nachweisen. 42,3\% der Frauen gaben an, im Laufe ihres Lebens vergewaltigt worden zu sein.

Schäfer et al. (2000) untersuchten Patienten mit polyvalentem Substanzgebrauch hinsichtlich eines sexuellen Missbrauchs vor dem 16. Lebensjahr. 70\% der Frauen und $56 \%$ der Männer gaben bei einer weiten Missbrauchsdefinition an, sexuell missbraucht worden zu sein.

Auch die Art der Traumatisierungen unterscheidet sich bei den Geschlechtern (Kessler et al. 1995). Belästigung und sexueller Missbrauch sind häufiger bei Frauen, während Unfälle, Kriegstraumata und Bedrohungen mittels einer Waffe häufiger bei Männern auftreten. Selbst wenn Frauen dasselbe Trauma erleiden wie Männer, haben sie etwa das doppelte Risiko, die Symptome einer PTBS zu entwickeln und ihre Symptome neigen mehr dazu, zu persistieren (De Lisi et al. 2003; Kessler et al.1995; Stein 2002; Van Loey et al. 2003). Frauen erfahren nach Traumatisierungen mehr negative Reaktionen von Familie und Freunden, wodurch die Entwicklung einer PTBS begünstigt wird.

Die Geschlechterdifferenzen können sich allerdings im Rahmen extremer Traumatisierungen auflösen (Kang et al. 2005).

Schnieders et al. (2006) fanden für die Gruppe suchtkranker Patienten mit Missbrauchserfahrungen sowohl für das körperliche als auch für das psychische Befinden eine deutlich höhere Symptombelastung als für die Referenzgruppe ohne sexuelle Missbrauchserfahrungen. Die Analyse bezüglich des Einflusses geschlechtsspezifischer Unterschiede wies für die Skalen Unsicherheit im Sozialkontakt und phobische Angst signifikant höhere Werte bei Frauen auf. Das bekräftigt, was Salter (1988) im Hinblick auf die unterschiedlichen Copingmechanismen von 
Frauen und Männern nach Missbrauchserfahrungen beschrieben hat: Danach entwickeln Männer eher externalisierende Verarbeitungsformen. Dazu passen Befunde, nach denen Männer mit einer Suchterkrankung häufiger zusätzlich eine dissoziale Persönlichkeitsstörung haben als Frauen. Frauen hingegen reagieren nach Auffassung Salters (1988) eher internalisierend mit nach innen gerichteten Aggressionen in Form von Angst, Selbstzweifeln und Schuldgefühlen. Der Autor schlussfolgert, dass Frauen vermehrt zu Mitteln greifen, die Innen kurzfristig Entspannung verschaffen und einen "Lösungsweg“ darstellen, mit Angst, Depressionen, Selbstzweifeln und Schuldgefühlen besser umzugehen und dass somit der Suchtmittelgebrauch gerade bei Frauen, die in der Kindheit missbraucht wurden, zum Problem wird.

Im Gegensatz dazu berichten Kessler et al. (1995), dass in der National Comorbidity Survey die Frauen mit PTBS im Vergleich zu Männern niedrigere Raten an Alkoholmissbrauch oder -abhängigkeit zeigten (27,9 vs. $51,9 \%)$, Drogenmissbrauch oder -abhängigkeit (26,9 vs. $34,5 \%$ ), aber höhere Raten an Panikstörungen (12,6 vs. $7,3 \%)$ und Agoraphobien (22,4 vs. $16,1 \%)$ hatten.

\subsection{Zur Geschichte der methadongestützten Behandlung der Opiatabhängigkeit}

Es wurde eingangs beschrieben, dass es im ehemaligen Niedersächsischen Landeskrankenhaus Göttingen bereits seit Anfang 1994 Spezialstationen zur Entgiftung illegaler Drogen gibt. Es wurde somit früh begonnen, eine „warme“, d.h. Methadon gestützte Entgiftung anzubieten. Wie andere Therapien unterlag auch die Suchtbehandlung einem fortwährenden Wandel. Es wird daher nun die Geschichte einer methadongestützten Behandlung der Opiatabhängigkeit skizziert. Die Substitutionstherapie mit Methadon geht auf Dole und Nyswander (1965) zurück. Schon 1963 führten sie ihr erstes Projekt durch.

Die Eignung dieser Behandlungsform für eine große Zahl Heroinabhängiger wurde 1970 durch ein unabhängiges Komitee der Columbia-University School of Public Health and Administration Medicine in New York festgestellt: Keiner der im Programm befindlichen Patienten wurde während des Untersuchungszeitraums rück- 
fällig mit Heroin, fast zwei Drittel der Patienten waren wieder berufstätig oder wandten sich einer Ausbildung zu. Die Inhaftierungsquote sank erheblich von initial 91 auf zuletzt 12\% (Gearing 1970). Zudem zeigte sich, dass eine Stabilisierung der Methadondosis innerhalb von zwei Jahren erreicht werden konnte. Aufgrund dieser Erfolge wurde die Methadonbehandlung 1970 als eine zweckmäßige Behandlungsform der Heroinabhängigkeit durch die New York State Medical Society anerkannt. Daraufhin breiteten sich die Methadonprogramme in den USA rasch aus, allerdings häufig mit schlechter Organisation und unzureichender Konzeption, so dass die ursprünglichen Erfolgsquoten von Dole und Nyswander nicht wiederholt werden konnten. Einheitliche Mindestanforderungen und Behandlungsregelungen für die Methadonsubstitution wurden 1974 in den USA durch die Einführung der Narcotic Addict Treatment Act verankert.

In Europa wurde das erste Methadonprogramm in enger Anlehnung an das ursprüngliche Dole-Nyswander-Modell 1966 in Schweden gestartet (Gunne 1983), und auch von anderen europäischen Ländern wurde die Substitutionstherapie Ende der 60er bzw. im Laufe der 70er Jahre übernommen. Seit 1987 gibt es auch in Österreich die Methadonbehandlung für Heroinabhängige auf der Basis eines Erlass des Gesundheitsministeriums, welcher im Januar 1991 neu gefasst wurde (Eisenbach-Stangl und Uhl 1992; Loimer 1992).

Im Laufe der 70er Jahre wurden in einigen asiatischen Ländern (wie Laos, Hongkong) sowie in Kanada und Australien Substitutionstherapien mit Methadon eingeführt.

In der Bundesrepublik Deutschland ist die Substitution mit Methadon eine relativ junge Therapieform zur Behandlung Heroinabhängiger. Abgesehen von einem Modellversuch in Hannover (Krach und Peschke 1987) in den frühen 70er Jahren und einem nicht publizierten Projekt in Göttingen wurde die Methadon-Substitution in der Bundesrepublik Deutschland strikt abgelehnt. Erst 1988 startete das erste Substitutionsprogramm in Nordrhein-Westfalen.

1990 wurde in der Bundesrepublik Deutschland der Weg für die Methadonsubstitutionstherapie auf breiter vertragsärztlicher Basis geebnet. Infolgedessen kommen auf die Entzugsstationen in zunehmendem Maße entgiftungswillige Langzeitsubstituierte zu. Diese haben teilweise ausgesprochen niedrige Durchhalteraten. 
Sees et al. (2000) berichten über ein Methadonentzugsprogramm, bei dem bis zum Schluss 90\% aller Teilnehmer die Entgiftung abbrachen. Es bestehen sogar Zweifel daran, ob bei Langzeitsubstituierten die Methadonentgiftung ein realistisches Behandlungsziel sein kann (Gossop und Strang 1991; Latowsky 1996; Rosenbaum 1991).

Die Orientierung auf Abstinenz als entscheidendem und unmittelbar zu erreichendem Ziel bei der Behandlung suchtkranker Patienten prägte die Drogenarbeit in Deutschland bis Mitte der 80er Jahre. Inzwischen ist die Arbeit mehr ausgerichtet an den realen Problemen und Ressourcen der Abhängigen und zielt in Richtung "Schadensbegrenzung“ (harm reduction): Unter diesem Blickwinkel haben auch die Therapieabbrüche nach zwei oder drei Behandlungswochen für einen multimorbiden Drogenpatienten überlebenssichernde und vielleicht auch sekundärpräventive Funktion, und die Behandlung ist nicht in erster Linie auf Abstinenz ausgerichtet. Das Angebot eines niederschwelligen qualifizierten Drogenentzugs soll dazu dienen, insbesondere diejenigen Patienten therapeutisch zu erreichen, die eine fortgeschrittene Drogenkarriere aufweisen (Hoffmann et al. 1997), möglicherweise eine Komorbidität mit einer anderen psychiatrischen Erkrankung zeigen (Krausz 1995) und nicht nur aufgrund hohen Drucks innerhalb des Drogenhilfesystems zu einem substanzfreien Leben motiviert sind (Bonorden-Kleij et al. 1993 a; Bonorden-Kleij et al. 1993 b). In diesem Sinne dient auch das Konzept der Niederschwelligkeit der Schadensminderung (Behrendt et al. 1993). Die Suchterkrankung muss als chronische Krankheit gesehen werden. Wie andere chronische Leiden stellen sie ein langjähriges prozesshaftes Geschehen dar mit Höhen, Tiefen und auch stabilen Phasen in ihrem jeweiligen Verlauf. Je nach Erkrankungsstadium sollte eine individuelle, gestufte Zielhierarchisierung erfolgen (vgl. Abbildung 1 und Abbildung 2, S. 26), um ein individuell angepasstes therapeutisches Casemanagement zu etablieren und die Zahl der Behandlungsabbrüche möglichst gering zu halten, denn jeder Behandlungsabbruch stellt ein kritisches Ereignis im Krankheitsverlauf einer Drogenabhängigkeit dar, insbesondere dann, wenn als Therapieziel der Ausstieg angestrebt wurde. Der Behandlungsabbruch ist - wie bereits beschrieben - schon allein deshalb bedrohlich, weil im direkten Anschluss daran vital gefährdende Intoxikationen gehäuft auftreten. 


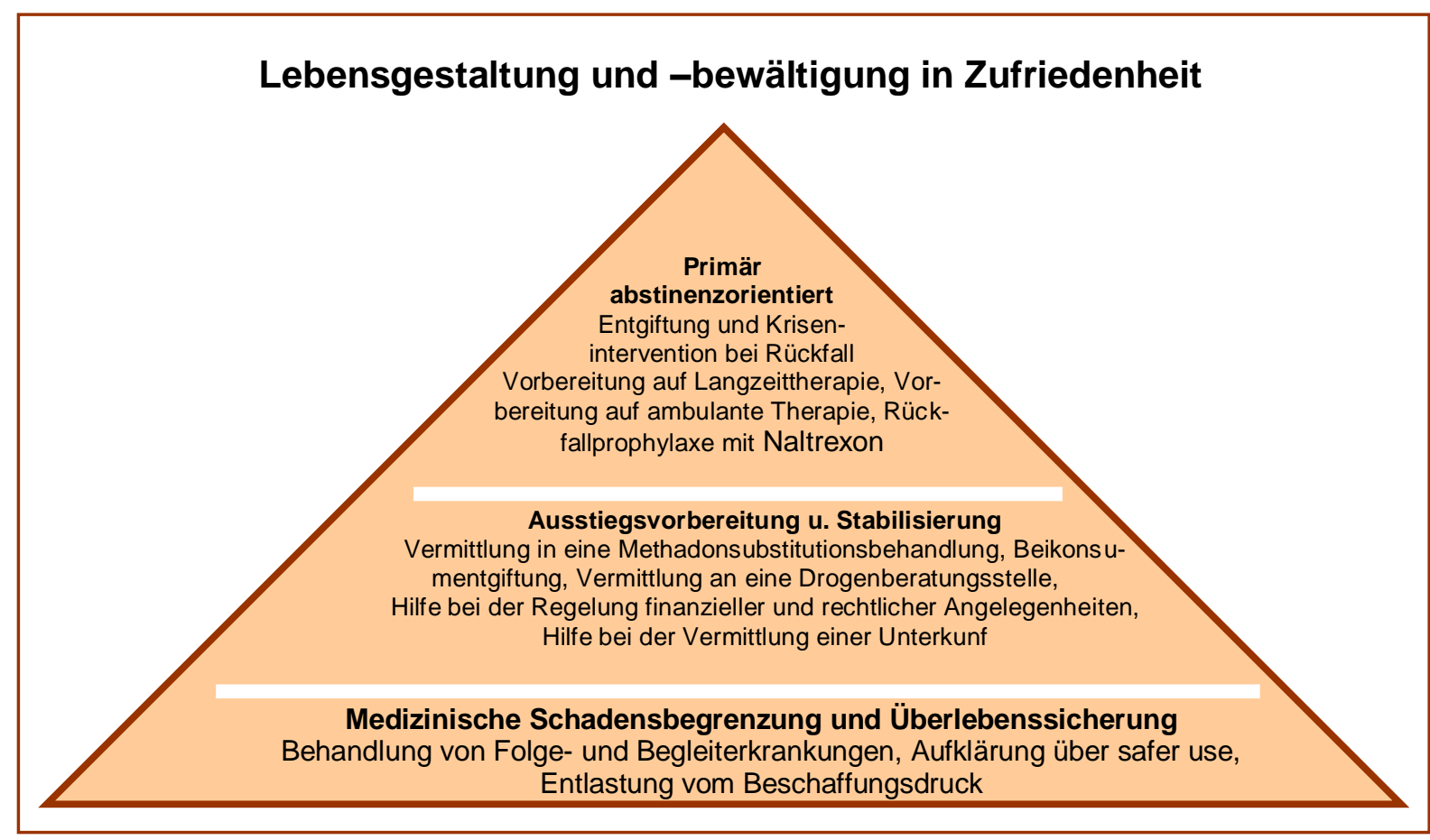

Abbildung 1: Zielpyramide der Drogenentzugsbehandlung mit niederschwelligem Zugangsweg (modifiziert nach Gößling 1997, S. 7)

Meili et al. (2004) schlagen eine Neuauflage der generellen Zielsetzung der Suchtbehandlung vor und verzichten auf die Abstinenz als Ziel (s. Abbildung 2)

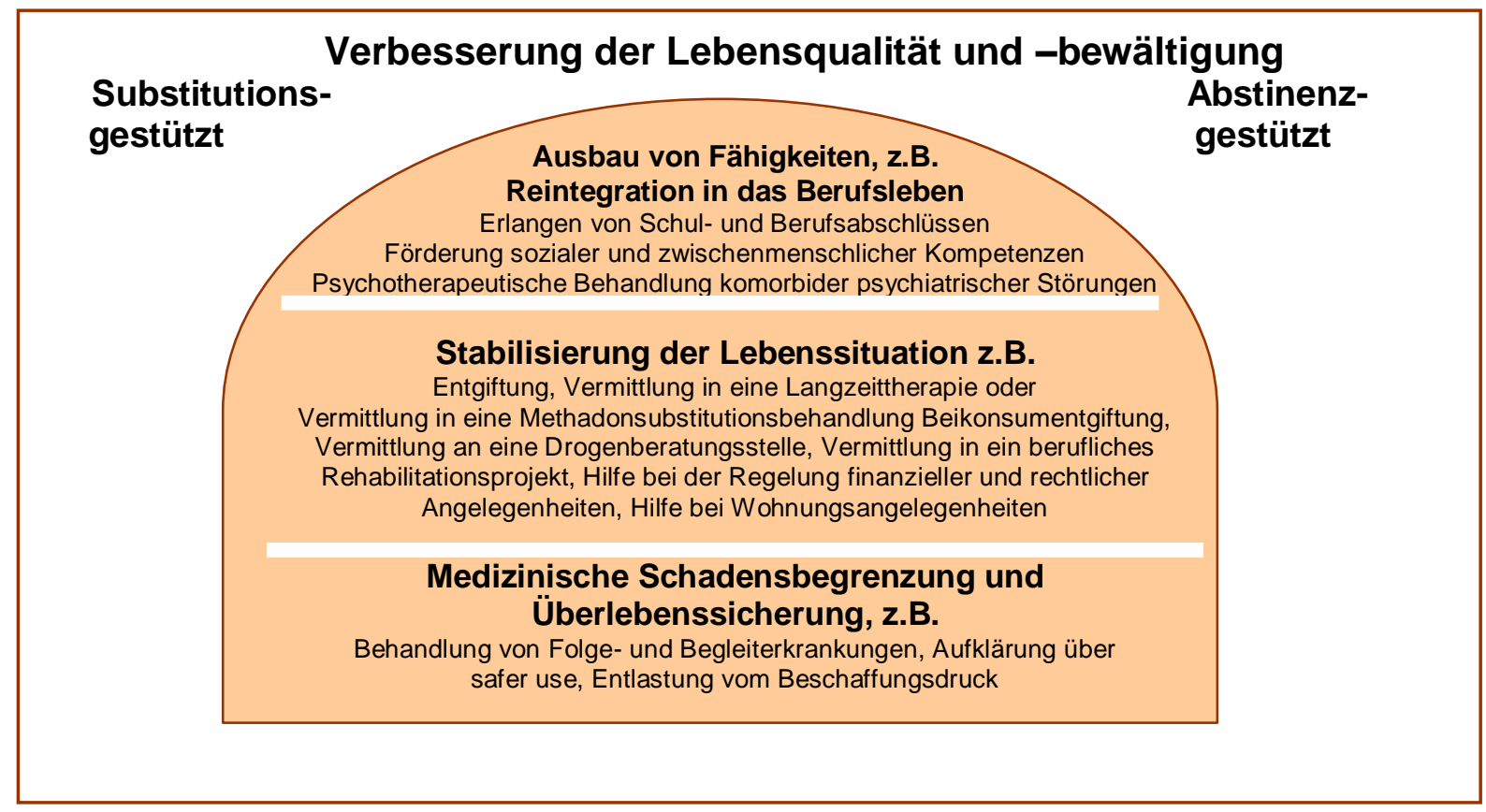

Abbildung 2: Zielsetzungen in der Suchttherapie jenseits des Abstinenzparadigmas (modifiziert nach Meili et al. 2004, S. 5) 
Es ist erwiesen, dass die Substitutionsbehandlung mit Methadon den Konsum illegaler Drogen reduziert, das Risiko einer HIV-Infektion verringert und sich positiv auf delinquentes Verhalten auswirkt (Marsch 1998; Mattick et al. 2003). Es gibt im klinischen Alltag jedoch immer wieder Patienten, die subjektiv mit dieser Therapieform unzufrieden sind bzw. von ihr nicht profitieren können. Diese Patienten klagen über belastende Nebenwirkungen (Müdigkeit, Gewichtszunahme und Ödeme (Longwell et al. 1979), Adynamie, Sexualstörungen, permanente Abhängigkeit von Einrichtungen des Suchthilfesystems) oder unzureichende Wirkung (fortgesetztes Suchtverlangen) der Substitutionstherapie. Daneben sind die Therapieverläufe in einer Reihe von Fällen wegen fortgesetzten Beigebrauchs von Alkohol (Backmund et al. 2003), Benzodiazepinen (Backmund et al. 2005) und/oder illegalen Drogen unbefriedigend (Hartel et al. 1995). Seit Einführung der Substitutionstherapie mit Levomethadon bzw. d-, I-Methadon Ende der 80er Jahre in Deutschland sind neben heroinabhängigen zunehmend auch Methadon behandelte Opioidabhängige zur stationären Entgiftungsbehandlung gekommen, um vom Beikonsum zu entgiften oder auch um die Substitutionstherapie zu beenden.

Der Benzodiazepinbeikonsum unter Opiatabhängigen ist ein weltweites Problem. Bei Methadonsubstituierten wurden Prävalenzraten bis $50 \%$ beobachtet, selbst bei Patienten mit jahrelanger kontinuierlicher Behandlung (Backmund et al. 2005; Bertschy 1995; Iguchi et al. 1993; Ministerium für Arbeit Gesundheit und Soziales des Landes Nordrhein-Westfalen 1998). Für täglichen bzw. abhängigen Konsum von Benzodiazepinen wurden Querschnittsraten zwischen 27\% und 45\% ermittelt (Backmund et al. 2005; Darke et al. 1993; Raschke 1994 S. 183). Durch die Sedierung sinken die Möglichkeiten zur Therapie und sozialen Rehabilitation, gleichzeitig gefährden sich die Patienten, und ein erhöhtes Risiko des Drogentodes wird bei ihnen diskutiert (Tretter 2001).

Außerdem existieren hohe Konsumraten von Kokain in Substitutionsbehandlungen. Leri et al. (2003) geben den Anteil der Kokainkonsumenten mit 50-60\% an, Dobler-Mikola et al. (2000) sprechen von 57\% Kokainkonsumenten unter den schweizer Substituierten. 
Madelung et al. (2006) konnten in einer Untersuchung an drogenabhängigen Patienten in Österreich zeigen, dass bei einer Substitutionstherapie mit Morphin im Vergleich zu Methadon ein reduzierter Zusatzkonsum von Heroin, Kokain und Benzodiazepinen auftritt. Es ist bekannt, dass ca. 30\% der methadonsubstituierten Patienten ein Alkoholproblem haben oder aber Alkoholiker sind (Helander et al. 1999; Hunt DE et al. 1986; Stastney und Potter 1991). Unklar ist, ob diese Patienten bereits Alkoholprobleme vor Beginn der Substitutionsbehandlung mitbrachten. Es gibt jedoch Hinweise darauf, dass sich im Verlauf der Substitutionsbehandlung die Anzahl der alkoholabstinenten Patienten erhöht (Vogt et al. 2000). Zusätzlich zum Risiko der Überdosierungen führt der fortgesetzte Alkoholismus langfristig zu erheblichen gesundheitlichen Folgeschäden (Gutjahr et al. 2001). Substituierte konsumieren signifikant mehr Alkohol als Heroinkonsumenten. Beigebrauch von Alkohol korreliert mit männlichem Geschlecht, längerer Dauer der Drogenabhängigkeit, täglichem Cannabiskonsum, und täglichem Benzodiazepinkonsum (Backmund et al. 2003). Persistierender Nebenkonsum im Rahmen einer Substitutionsbehandlung ist mit den Substitutionsrichtlinien nicht vereinbar und birgt zudem ein erhebliches Risiko an Mischintoxikationen und damit verbundener Letalitlät, so dass schließlich eine Beendigung der Substitutionstherapie resultiert.

Auf den Entgiftungsstationen hat die Zahl älterer, teilweise multimorbider langzeitsubstituierter Patienten zugenommen. Daneben gibt es unter den Jugendlichen und jungen Erwachsenen einen Trend im Konsummuster mit Zunahme des Gebrauchs von Cannabis, Amphetaminen, Ecstasy und Kokain (Kraus et al. 2005). Das Durchschnittsalter der Opioidabhängigen auf den Entgiftungsstationen hat sich demzufolge seit Anfang der 90er Jahre nach oben verschoben (Backmund et al. 2006), polyvalenter Substanzkonsum inklusive einer Opioidabhängigkeit stellt eine häufige Diagnose auf Entgiftungsstationen dar, reine Opiatentgiftungen sind seltener geworden.

Angesichts der aufgeführten breit gefächerten Problematik bei der Behandlung opioidabhängiger Menschen, ist es nicht verwunderlich, dass in einer großen Anzahl von Evaluationsstudien nachgewiesen wurde, dass es eine Einheitsbehandlung nicht geben kann. Ein gutes Behandlungssystem muss individuell dem jewei- 
ligen Fall angepasst sein und auf verschiedene Behandlungsmodalitäten zurückgreifen können.

Durch Veränderungen der Zielsetzungen im Rahmen der Suchtbehandlung von der primär abstinenzorientierten Behandlung hin zu Konzepten der Schadensbegrenzung, Niederschwelligkeit und langfristigen Stabilisierung über Vermittlung der Patienten in Substitutionsprogramme einerseits, sowie andererseits durch zunehmenden ökonomische Druck, haben sich in den letzten Jahren einige Veränderungen in der Suchttherapie abgezeichnet: In der stationären Entgiftungsbehandlung zeigt sich ein Trend mit Zunahme der Entgiftung des Nebenkonsums bei laufender Substitutionsbehandlung. Bei längerer Überlebenszeit suchtkranker methadonsubstituierter Patienten steigt das Durchschnittsalter der Patienten auf Entgiftungsstationen und eine Zunahme schwerer somatischer Folgekrankheiten jahrelanger Alkohol- und Drogenabhängigkeit ist zu verzeichnen.

Auch Krausz (2004) bezieht sich auf diese Entwicklung bei seinem Entwurf von " Paradigmen und Prinzipien der Suchtbehandlung von morgen“, und meint: „Durch den ökonomischen Druck werden Ausstattung und Behandlungszeiten infrage gestellt, durch schwere somatische Folgekrankheiten entsteht Handlungsdruck genauso wie durch den wissenschaftlichen Beleg der Effektivität neuer Interventionen (kontrolliertes Trinken, motivierende Gesprächsführung, BuprenorphinSubstitution u.a.)“ (Krausz 2004, S. 18). Er schlussfolgert: „Weder der Abbau von Behandlungsressourcen im stationären Bereich noch die reine Kürzung von Behandlungszeiten haben irgendeinen Sinn für die Betroffenen. Die Mittel in diesen Bereich sind dringend nötig für eine qualifizierte Suchttherapie, die aber im Sinne einer effektiveren Breitenwirkung neuer innovativer, integrierter Therapieangebote nutzbar gemacht werden müssen“, (Krausz 2004, S. 19). Die vorliegende Arbeit leistet ihren Beitrag eben dazu.

\section{4 Übersicht der Literatur zum Einsatz der Videografie in der Psychiatrie und Psychotherapie}

Mit zunehmender Verbreitung der Videotechnik in den 60er und 70er Jahren erschienen schon damals zahlreiche Arbeiten vorwiegend aus dem angloamerikani- 
schen Raum zur Anwendung der Videografie in der Psychiatrie und Psychotherapie, darunter deren Einsatz bei Gruppentherapien (Alger und Hogan 1967; Stoller 1969), Paartherapien (Alger und Hogan 1969; Eisler et al. 1973), im Psychodrama (Goldfield und Levy 1968) und bei der Behandlung alkoholkranker Patienten (Baker et al. 1975; Paredes et al. 1969).

Die Videografie wird im Rahmen der Psychiatrie und Psychotherapie sowohl zu diagnostischen Zwecken (wie zum Beispiel zur Abklärung psychogener Krampfanfälle) (Harden et al. 2003) als auch zum Monitoring therapeutischer Prozesse (Roscher 2006) und zur Aus- und Weiterbildung (Ekblad et al. 2004; Handmaker et al.1999; Walter et al. 2004) sowie zur Supervision (Abbass 2004) eingesetzt. AuBerdem wird sie zu Forschungszwecken bei verschiedenen Krankheitsbildern wie schizophrenen Psychosen (Angermeyer et al. 2003), Störungen der Mutter-KindInteraktion (Beebe 2005), Spinnenphobie (Johanson et al. 2006), Alkoholkrankheit (Seppä et al. 2004), Creutzfeldt-Jakob-Krankheit (Landolt et al. 2006), Alexithymie (Rasting et al. 2005), Anorexia nervosa (Probst et al. 1990) oder Bulimie (Stein et al. 2006) genutzt. Darüber hinaus findet sie Anwendung in der psychiatrischen Therapie beispielsweise bei autistischen oder hyperaktiven Kindern (Chung et al. 2006; Furman und Feighner 1973), bei postpartalen Störungen (Hornstein et al. 2006) bei sozialer Phobie (Harvey et al. 2000; Kim et al. 2002; Rapee und Hayman 1996), oder der Alkoholerkrankung (Baker et al. 1975; Paredes et al. 1969). Videofeedback wurde vor dem Hintergrund verschiedener Zielvorstellungen als therapeutisches Mittel in der Psychiatrie eingesetzt: So wollte man beispielsweise den Widerstand in der Behandlungssituation vermindern, die Behandlungsmotivation stärken (Griffiths 1974), das Auftreten maladaptiven Verhaltens verringern und akzeptableres Verhalten einüben, Informationen über Diskrepanzen zwischen Ist-Zustand und Zielzustand gewinnen und bearbeiten (Lincoln 1956) und Verhaltensänderungen durch Abschreckung bei alkoholkranken Patienten (Paredes et al. 1969) oder durch Korrektur einer gestörten Selbstwahrnehmung beim Sozialphobiker (Harvey et al. 2000) bewirken. Boyd und Sisney (1967) zeigten, dass Videofeedback zu erhöhter Selbstakzeptanz führen kann. Geertsma und Reivich (1965) wiesen Effekte von Selbstkonfrontation auf eine realistischere Selbstbewertung nach, Moore et al. (1965) fanden bei Patienten, die Videofeed- 
back bekommen hatten, eine stärkere Verbesserung der klinischen Symptomatik als in der Kontrollgruppe. Die Wirkmechanismen, die den Effekten des Videofeedback zugrunde liegen, wurden schon früh mit verschiedenen theoretischen Modellen in Zusammenhang gebracht (Griffiths 1974) z.B. mit dem Fertigkeitenmodell, den Imitationsprozessen sozialen Lernens sowie der Dissonanztheorie (Festinger 1957). Es ist mehrfach dokumentiert worden, dass auch Stress und Angst durch eine videogestützte Selbstkonfrontation ausgelöst werden (Bailey und Sowder 1970; Feinstein und Tamerin 1972; Schaefer et. al. 1971). Einige Autoren schlussfolgern auch, dass schädliche bzw. negative Wirkungen durch Videokonfrontation (Bailey und Sowder 1970; Danet 1968; Schaefer et al. 1971) auftreten können. Immer wieder wird hervorgehoben, dass die Videografie allein bzw. das Videofeedback allein nur von geringem Nutzen ist. Positive therapeutische Effekte bedürfen einer Kopplung von Videofeedback mit therapeutischen Instruktionen (Baker et al. 1975; Eisler et al. 1973; Harvey et al. 2000; Kim et al. 2002; Rapee und Hayman 1996). Detailliertes Feedback erwies sich als effektiver als Feedback in einer generalisierten Form, wie "richtig“ oder "falsch“ (McGuigan 1959). Mehrdeutiges Feedback ist zu vermeiden (Hunt DP 1964). Bilodeau und Bilodeau (1969) kommen zu dem Schluss, dass das Feedback sofort und nicht verzögert erfolgen sollte, um die Effektivität zu steigern. Es wird von den Autoren daher für sinnvoll gehalten, ein laufendes Replay zu unterbrechen, um sofort Kommentare zu geben.

Auch Harvey et al. (2000) fanden in einer Studie zur sozialen Phobie, dass kognitive Vorbereitung den therapeutischen Effekt von Video Feedback erhöht. Kim et al. (2002) wiesen eine Generalisierung der durch kognitive Vorbereitung erreichten Effekte bei einer zweiten Exposition nach.

Insgesamt wird deutlich, dass hinsichtlich des Settings sowohl für die Situation der Videoaufnahme als auch für die Replaysituation zahlreiche Varianten existieren. Das führt dazu, dass die verschiedenen Studien oft nicht direkt vergleichbar sind. Ein weiterer Aspekt, der die Vergleichbarkeit erschwert, besteht darin, dass Videofeedback teilweise mit anderen Formen der Behandlung kombiniert wird, manchmal aber auch nicht. Sollte dies der Fall sein, so ist schwerlich herauszufinden, ob die festgestellten Effekte für das Videofeedback spezifisch sind oder nicht. 
Einigkeit besteht weitgehend darüber, dass sich die meisten Patienten schnell an das künstliche Umfeld mit Mikrofon und Kamera gewöhnen, so dass Videokamera und Mikrophon in den meisten Studien deutlich sichtbar für die Probanden aufgestellt wurden. Nur bei wenigen älteren Untersuchungen wurden sie versteckt plaziert. Aspekte der Aufnahmetechnik werden von Jones (1972) und Berger (1970) diskutiert.

Das Videofeedback in der klinischen Situation beinhaltet zwei Formen des Feedbacks: 1. Das Playback erlaubt dem Einzelnen, sein eigenes Verhaltens anzusehen und mögliche Effekte seines Verhaltens zu beobachten. 2. Weiterhin ermöglicht die Videofeedback-Situation auch eine Erörterung durch den Therapeuten.

Die Feedbacksitzung kann dabei ganz unterschiedlich gestaltet werden. Alger beschreibt 1969 als gängige Methode, ein kurzes Stück einer Therapiesitzung (10 - 15 Minuten) aufzunehmen und es gleich im Anschluss anzusehen und so fort. Ein anderes Verfahren besteht darin, eine ganze Sitzung aufzunehmen und eine weitere separate Replay-Sitzung zu haben (z.B. Berger et al. 1968). Ein weiteres Vorgehen wurde in der Gruppentherapie beschrieben: Eine Videoaufnahme wird hier während einer regulären Sitzung gemacht und gestoppt, wenn ein Teilnehmer einschl. Therapeut danach fragt, eine spezielle Passage noch einmal anzusehen. Moore et al. (1965) beschreiben eine Methode, bei der nacheinander Videosegmente angeschaut werden, die über einen längeren Zeitraum entstanden sind. Dabei wird ein Vorteil darin gesehen, dass erhebliche Änderungen des Verhaltens und auch des Erscheinungsbildes dem Rezipienten deutlich gemacht werden können. Cornelison und Tausig (1964) entwickelten eine direkte Konfrontationsmethode, wobei die Probanden ihr eigenes Gesicht nahezu in Lebensgröße schon im Moment der Videoaufnahme auf einem Monitor anschauen können sowie auch später noch einmal im Rahmen einer Playbacksitzung. Bei einer anderen Vorgehensweise wird das Playback in nur einem Modus (nur der Ton oder nur das Bild) vorgeführt. Dies könnte helfen, die Aufmerksamkeit auf bisher vernachlässigte Aspekte der Kommunikation zu lenken. Beschrieben wurden des weiteren Replayverfahren mit im Vergleich zur Aufnahmesituation veränderter Geschwindigkeit, veränderter Bild- oder Tonqualität. Das soll dem Rezipienten u.a. ermögli- 
chen, mehr Distanz zu gewinnen und einen Perspektivenwechsel vorzunehmen (Alger 1969).

Während der Replaysituation kann das Material vom Patienten und Therapeuten unkommentiert angeschaut und im Anschluss besprochen werden. Das Video kann unter bestimmten Gesichtspunkten oder auch ohne fokussierende Instruktionen des Therapeuten angeschaut werden. Werden Kommentare während der Replaysitzung abgegeben kann das Videoband weiterlaufen oder gestoppt werden. Die Haltung des Therapeuten kann zurückhaltend sein oder aktiv. Patienten können instruiert werden, frei zu kommentieren oder das Augenmerk auf Verhaltesoder Gefühlsaspekte zu legen. Sie können auch aufgefordert werden, auf Diskrepanzen zwischen Verhalten und Gefühlen in der Aufnahmesituation und ihren aktuellen Gefühlen zu achten.

Die Menge an Feedback variiert von einzelnen Sitzungen Griffiths und Hinkson (1973) bis zu Serien von Videoaufnahmen, die über mehrere Wochen aufgenommen wurden (Bailey 1970). In der Realität des klinischen Alltags wird die Menge des zu ermöglichenden Feedbacks schon allein durch die Anforderung hoher Effizienz im Arbeitsalltag mit immer kürzeren stationären Aufenthaltsdauern sowie steigendem Druck durch die Kostenträger begrenzt. 


\subsection{Furchtappelle als Mittel zur Einstellungs- und Verhal- tensänderung}

Im Folgenden wird zunächst der Frage nachgegangen, was genau unter einem Furchtappell zu verstehen ist. An diese Begriffsbestimmung schließen sich die Darstellung klassischer sowie neuer Theorien zur Informationsverarbeitung persuasiver ${ }^{1}$ Botschaften an.

\section{Begriffsbestimmung}

Barth und Bengel (1998, S.51) definieren „Furchtappelle“ wie folgt:

„Unter Furchtappellen werden persuasive Botschaften verstanden, welche dem Empfänger mitteilen, dass für inn relevante Werte (wie Leben, Gesundheit, Eigentum etc.) bedroht sind. Furchtappelle bestehen aus verbalem oder nonverbalem Material, welches beim Empfänger Furcht auslösen und hierdurch Verhaltens- und Einstellungsänderungen bewirken sollen."

Bei der Furchtinduktion handelt es sich um eine der am häufigsten gebrauchten Techniken zum Zwecke der Einstellungsänderung (Aronson et al. 2002). Einstellungen haben nach Triandis (1971) kognitive, affektive und handlungsorientierte Komponenten und werden als handlungsleitend interpretiert. Rosenberg und Hovland (1960), (siehe Abbildung 3, S. 35 haben Einstellungen durch messbare Effekte auf diesen drei Ebenen definiert.

\footnotetext{
${ }^{1}$ Der in englischsprachigen Veröffentlichungen gebrauchte Begriff „persuasion“ meint Überredung und wird nach Simons (1986) folgendermaßen definiert: „Überredung ist eine Form der Beeinflussung, die prädisponiert aber nicht aufzwingt. Sie verändert die Bewertungen des Anderen und nicht nur sein Verhalten. Sie beeinflusst seine Empfindungen dafür, was falsch oder richtig, wahrscheinlich oder unwahrscheinlich ist; seine Bewertung von Personen, Ereignissen, Ideen, Vorschlägen, seine privaten oder öffentlichen Verpflichtungen, diese oder jene Dinge zu tun; möglicherweise auch die Grundwerte oder Ideologien“. (zitiert aus Simons 1986, S.22; übersetzt durch die Verfasserin)
} 


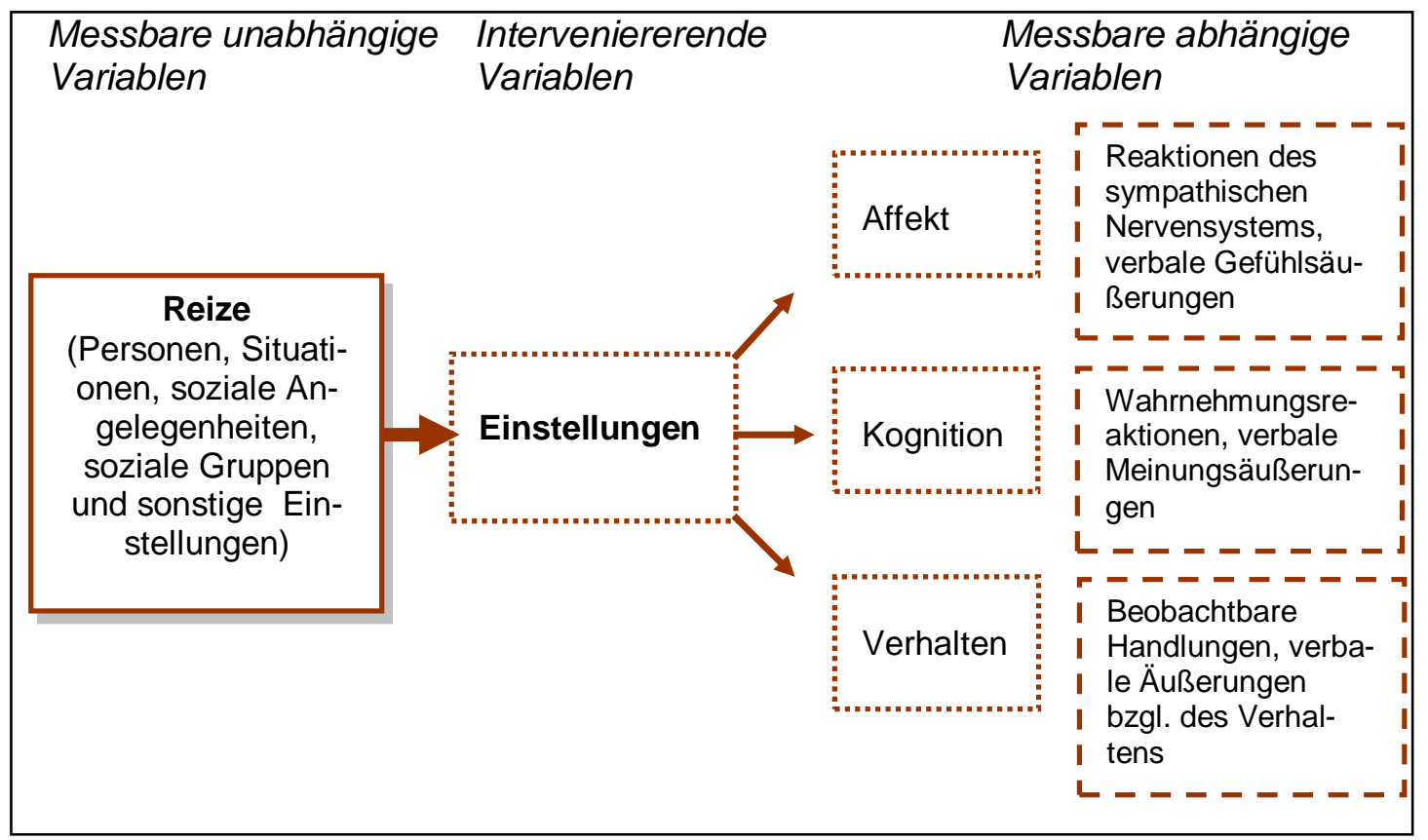

\section{Abbildung 3: Dreikomponentenmodell der Einstellung nach Rosenberg}

und Hovland (zitiert aus Rosenberg und Hovland 1960, S. 3)

Die Furchtappellwirkung ist ein komplexer und äußerst komplizierter Prozess der inneren Verarbeitung (siehe Abbildung 4, S. 35), welcher nach wie vor nicht vollständig geklärt ist. Der Furchtappell muss dazu wahrgenommen und enkodiert, d.h. in eine mentale Repräsentation übersetzt werden, welche im Gedächtnis abgelegt wird. Interferenzen werden gebildet, d.h. die Information wird interpretiert, mit bereits vorhandenem Wissen verknüpft und beurteilt. Sie führt am Ende möglicherweise zu einem bestimmten Folgeverhalten.

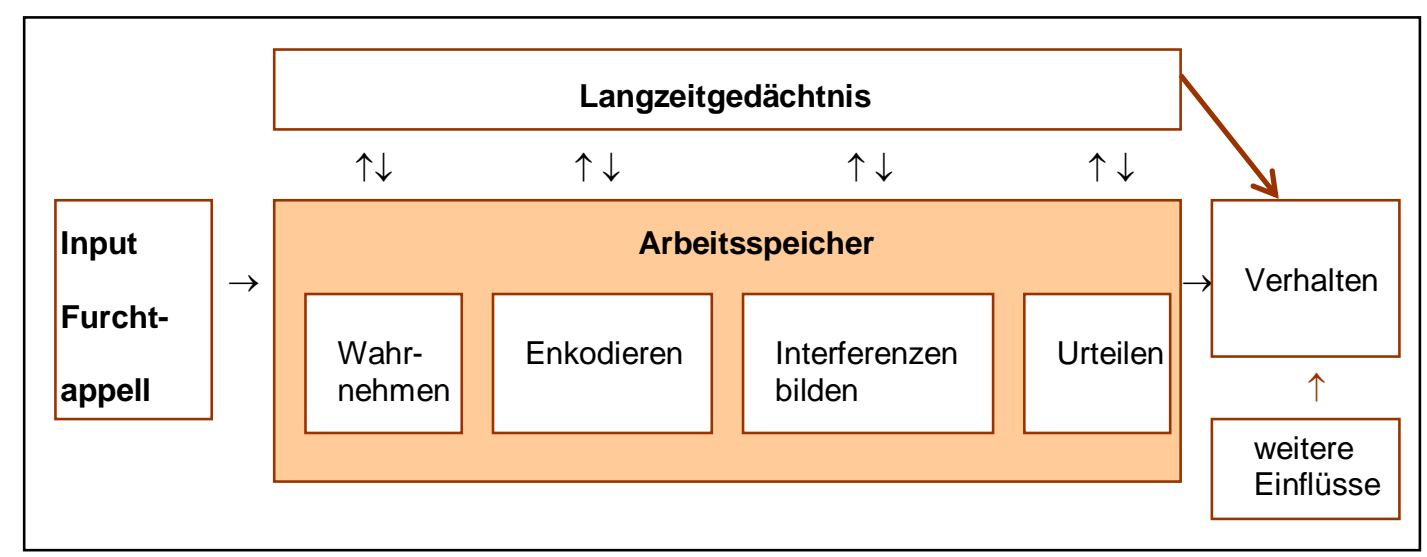

Abbildung 4: Prozess der Informationsverarbeitung

(modifiziert nach Wänke 2002, S. 484-485) 
Petty et al. (1991) gehen davon aus, dass das Ausmaß und die Richtung der Einstellungs- und vor allem der Verhaltensänderung von der Art der Verarbeitung einer präventiven Botschaft abhängen. Um die möglichen Arten der Verarbeitung zu verdeutlichen werden sechs Empfänger und deren Verhaltensänderungen auf einen Werbespot gegen Drogenkonsum dargestellt (siehe Abbildung 5, S. 36):

\begin{tabular}{|c|c|c|c|c|c|c|}
\hline & Person A & Person B & Person C & Person D & Person E & Person F \\
\hline Wissen & nichts & \multicolumn{4}{|c|}{$\begin{array}{l}\text { Einige Personen, die Marihuana rauchen, wer- } \\
\text { den später harte Drogen nehmen und sind } \\
\text { in Gefahr, ihr Leben zu zerstören. }\end{array}$} & $\begin{array}{l}\text { Eine be- } \\
\text { rühmte } \\
\text { Person } \\
\text { sagt, man } \\
\text { solle „nein“ } \\
\text { zu Drogen } \\
\text { sagen. }\end{array}$ \\
\hline \multirow[t]{2}{*}{$\begin{array}{l}\text { Kognitive } \\
\text { Reaktion } \\
\text { auf die } \\
\text { Botschaft }\end{array}$} & $\begin{array}{l}\text { (irrele- } \\
\text { vant) }\end{array}$ & $\begin{array}{l}\text { Aber eini- } \\
\text { ge Men- } \\
\text { schen- } \\
\text { möchten } \\
\text { dies }\end{array}$ & $\begin{array}{l}\text { Marihua- } \\
\text { na ist } \\
\text { gefährlich } \\
\text { für andere } \\
\text { Men- } \\
\text { schen }\end{array}$ & \multicolumn{2}{|c|}{$\begin{array}{l}\text { Marihuana kann für } \\
\text { mich gefährlich sein }\end{array}$} & $\begin{array}{l}\text { Die be- } \\
\text { rühmte } \\
\text { Person rät } \\
\text { vom Dro- } \\
\text { genkonsum } \\
\text { ab }\end{array}$ \\
\hline & & \multicolumn{2}{|c|}{$\begin{array}{l}\text { Die Nachricht ist für } \\
\text { mich nicht von Rele- } \\
\text { vanz }\end{array}$} & $\begin{array}{l}\text { Ich mag } \\
\text { Gefahr }\end{array}$ & $\begin{array}{l}\text { Ich mag } \\
\text { keine } \\
\text { Gefahr }\end{array}$ & $\begin{array}{l}\text { Ich mag die } \\
\text { Person }\end{array}$ \\
\hline Einstellung & & & & $\begin{array}{l}\text { lch könn- } \\
\text { te Drogen } \\
\text { mögen }\end{array}$ & $\begin{array}{l}\text { Ich mag } \\
\text { keine } \\
\text { Drogen }\end{array}$ & $\begin{array}{l}\text { Ich mag } \\
\text { keine Dro- } \\
\text { gen }\end{array}$ \\
\hline Verhalten & & & & $\begin{array}{l}\text { Mögliche } \\
\text { Einnahme } \\
\text { von Dro- } \\
\text { gen }\end{array}$ & $\begin{array}{l}\text { Abstinenz } \\
\text { von Dro- } \\
\text { gen }\end{array}$ & \\
\hline
\end{tabular}

Abbildung 5: Mögliche Antworten auf den Werbespot „Just say no“ (zitiert aus Petty et al. 1991, S. 75; übersetzt durch die Verfasserin )

„Person A versteht die Nachricht nicht, so dass keine weitere Informationsverarbeitung erfolgt.

Die Personen B, C, D und E verstehen die Botschaft, entwickeln nachfolgend jedoch unterschiedliche Gedanken, wobei lediglich Person D und E in die gewünschte Richtung denken („Drogen sind gefährlich"). 
Für Person D, die das Selbstbild hat, besonders risikofreudig zu sein, ist aber gerade diese Einschätzung ein Grund mehr, um in Zukunft Drogen zu konsumieren, da dieses Verhalten in ihr Selbstkonzept passt.

Nur bei Person E entsteht letztlich der Wunsch, der Gefahr zu entgehen, verbunden mit der Motivation keine Drogen zu nehmen.

Person $\mathrm{F}$ versteht die Nachricht wegen mangelnder kognitiver Fähigkeiten nicht, ändert aber ihre Einstellung aufgrund eines irrelevanten Botschaftsmerkmals, nämlich der Sympathie für den Sprecher. Diese Einstellungsänderung hat jedoch für ihr Verhalten keine Konsequenz" (Petty et al. 1991, S.74; übersetzt durch die Verfasserin)

Als wichtigstes Resultat arbeiten die Autoren heraus, dass es neben der erwünschten einen Form der Verarbeitung noch fünf andere Arten der Interpretation einer Nachricht gibt, welche nicht zur erwünschten Verhaltensänderung führen. Der Informationsverarbeitung kommt demnach eine entscheidende Rolle bei der Wirkung eines Furchtappells zu. Im Folgenden wird sie daher ausführlich betrachtet.

\subsubsection{Die Rolle der Informationsverarbeitung im Rahmen ei- nes Furchtappells}

Es werden nun sowohl die klassischen als auch neuere Theorien zur Informationsverarbeitung im Rahmen eines Furchtappells dargestellt.

Die Informationsverarbeitung, die einer Vielzahl an Einfluss nehmenden Faktoren unterliegt, kann im Allgemeinen an jeder Stelle abgebrochen und später wieder aufgenommen werden. Sie wird durch die Gedächtnisleistungen aber auch durch andere Faktoren wie z.B. soziale Bedingungen beeinflusst. Auch das Selbstbild kann die Informationsverarbeitung verändern, beispielsweise durch Verzerrungstendenzen oder Selektionsprozesse. Furchtappelle stehen inhaltlich in Konflikt zum positiven Selbstbild - dieses kann kognitive Dissonanzen erzeugen (Aronson 
et al. 2002), welche Menschen zu Veränderungen auf einer Einstellungsebene motivieren.

\section{Die Dissonanztheorie nach Festinger (1957)}

Die Dissonanztheorie geht ursprünglich auf Festinger (1957) zurück. Sie baut darauf auf, dass Menschen nach Konsistenz in Bezug auf ihre Kognitionen, ihr Handeln und ihre Erfahrungen streben. Bei Konfrontation mit inkonsistenten Kognitionen werden sogenannte Dissonanzen erlebt - aversive motivationale Zustände, welche nach Linderung verlangen. Furchtappelle können also dazu dienen, möglichst viel Dissonanz auszulösen, um eine möglichst starke Handlungsmotivation zu bedingen. Ziel ist es, dass die Empfänger der Nachricht durch Anpassung ihres Verhaltens an die dissonante Kognition eine Dissonanzreduktion bewirken. Es gibt jedoch auch diverse andere Möglichkeiten, diese zu erreichen: Zum Beispiel durch Änderung der Kognition statt durch Änderung des Verhaltens, durch Abwertung der Quelle der Botschaft, Verleugnung der angesprochenen Gesundheitsgefahren, durch das Anfügen konsonanter Kognitionen ("Drogen wirken entspannend") oder durch eine Bestätigung des Selbst durch die Betonung positiver Dimensionen, die von der Selbstbedrohung unabhängig sind (,ich nehme zwar Drogen, aber dafür bin ich ein guter Sportler"). Die Dissonanztheorie spezifiziert nicht näher, welche der zahlreichen Möglichkeiten zur Dissonanzbewältigung wann einsetzen. Menschen sind jedoch bestrebt, auf derjenigen Ebene Veränderungen vorzunehmen, die ihnen am leichtesten erscheint. Somit lässt sich von einer hohen Dissonanz nicht direkt auf eine Verhaltensänderung schließen, sie macht diese aber wahrscheinlicher. Konkret vorgegebene Bewältigungsempfehlungen in einer persönlichen Botschaft begünstigen die Dissonanzbewältigung auf dem erwünschten Weg, da Verhaltensalternativen nun vorgegeben werden und somit Änderungen auf der Verhaltensebene erleichtert werden.

\section{Das „Elaboration Likelihood Model“ von Petty und Cacioppo (1986)}

Nahe liegend ist weiterhin, dass Furchtappelle eine Stimmungsinduktion bewirken. Gut belegt ist ein allgemeiner Einfluss der Stimmung auf die Prozesse der Informationsverarbeitung (Bless 1997; Clore et al. 1994; Schwarz 1990; Schwarz u. 
Bless 1991). Es wird davon ausgegangen, dass die bei einem Furchtappell entstehende Furchterregung meist als unangenehmer emotionaler Zustand empfunden wird, der zur Aktivierung von kognitiven, affektiven und Verhaltens-Reaktionen führt, mit dem Ziel, den aversiv erlebten Zustand zu beenden bzw. zu lindern.

Das „Elaboration Likelihood Model“ von Petty und Cacioppo (1986) und das „Heuristic systematic Model“" von Chaiken et al. (1989) sind neuere Theorien zur Erklärung von Einstellungsänderung durch persuasive Botschaften. Sie zeigen, dass Personen in schlechter Stimmung persuasive Botschaften eher systematisch und analytisch unter Berücksichtigung der Qualität der Argumente verarbeiten, wohingegen Personen in guter Stimmung eher auf Heuristiken, Stereotypien und Skripts zurückgreifen und die Qualität von Persuasionsargumenten weniger genau beachten (Chaiken et al. 1989). Petty und Cacioppo (1986) bezeichnen diese Prozesse als "Verarbeitung auf zentralem bzw. peripherem Weg“.

Eine Einstellungsänderung auf zentralem Weg bzw. auf systematische Weise scheint stabiler und resistenter gegen Gegenargumente zu sein. Verhalten kann hier eher vorhergesagt werden als bei einer Einstellungsänderung auf peripherem Weg. Eine systematische Verarbeitung ist somit wünschenswert.

Nach dem Triebreduktionsmodell (Dollard und Miller 1950) hat Furcht als unangenehme Emotion die Eigenschaft eines (An-)Triebs. Die durch den Sender einer Nachricht induzierte Furcht motiviere demnach den Empfänger zu Verhaltensweisen, die zu einer Furchtreduktion führen. Das Verhalten ist nach Ansicht der Autoren nicht zielgerichtet sondern funktioniere nach dem Prinzip „trial and error“. Jede Reaktion, die zu einer Furchtreduktion führt, wird also verstärkt.

\section{Die kurvilineare Hypothese von Janis (1967)}

Die kurvilineare Hypothese von Janis (1967) samt seiner family of courves und McGuire's nonmonotonic models (1968 a, 1968 b) haben Annahmen dieser Theorie weiter entwickelt. Nach theoretischen Annahmen von Janis (1967) und McGuire (1968 a, 1968 b, 1978) entfalten Furchtappelle mittleren Ausmaßes die größte Wirkung. Sie beschrieben einen umgekehrt U-förmigen Zusammenhang zwischen der Stärke der Furcht und dem Ausmaß der Einstellungsänderung. Die Quantifizierung dessen, was unter einer mittleren Furchtstärke zu verstehen ist, 
konnte allerdings bisher empirisch nicht geleistet werden. Ebenfalls gelang keine empirische Spezifizierung, welches Furchtniveau maximal tolerabel ist, bevor es zu Abwehrprozessen kommt.

\section{Theorie der Schutzmotivation nach Rogers (1975/1983) sowie Furcht- und Gefahrenkontrolle nach Leventhal (1970)}

Im Rahmen der Weiterentwicklung der Theorien (beispielhaft Leventhal (1970), Leventhal und Hirschmann (1982), Leventhal und Watts (1966), Rogers (1975, 1983)) wird nicht mehr von einem kurvilinearen Zusammenhang von Furchtstärke und Einstellungsänderung ausgegangen, da sich häufig ein direkter positiver Zusammenhang zwischen Ausmaß der Einstellungsänderung und Furchtinduktion finden ließ. Wurden Erkrankungen als potentiell den Empfänger betreffend als relativ rasch eintretend und mit extremen Folgeschäden (tödlicher Verlauf) behaftet dargestellt, so zeigte sich eine höhere Inanspruchnahme medizinischer Vorsorgeuntersuchungen oder ein verändertes Gesundheitsverhalten im Sinne einer Reduktion riskanter Handlungen. Die Autoren unterschieden zwischen der wahrgenommenen Furcht vor einer gesundheitlichen Bedrohung und den wahrgenommenen Bewältigungsmöglichkeiten. Die beiden Prozesse sind meist unabhängig voneinander d.h. sie verlaufen parallel (Modell der parallelen Reaktionen), können einander jedoch beeinflussen. Nach dieser Theorie erscheint die Furchtinduktion als motivationaler Faktor für eine Verhaltensänderung sinnvoll, sollte jedoch auf jeden Fall gekoppelt sein mit der Vermittlung von Bewältigungsmöglichkeiten. Nach Rogers $(1975,1983)$ sind die wesentlichen Komponenten des Furchtappells: die wahrgenommene Schädlichkeit, Auftretenswahrscheinlichkeit (=Vulnerabilität), Wirksamkeit der Empfehlung zur Abwendung des Schadens, empfundene Selbstwirksamkeit und Reaktionskosten. Die Schutzmotivation als zentrales Konstrukt der Theorie Rogers (1975) ist ein wichtiger Vermittler zwischen Furchtappell und Verhalten und entspricht quasi der Intention für ein Verhalten. In Abbildung 6 (S.41) ist das revidierte Modell von Rogers in der Übersicht zu sehen. 


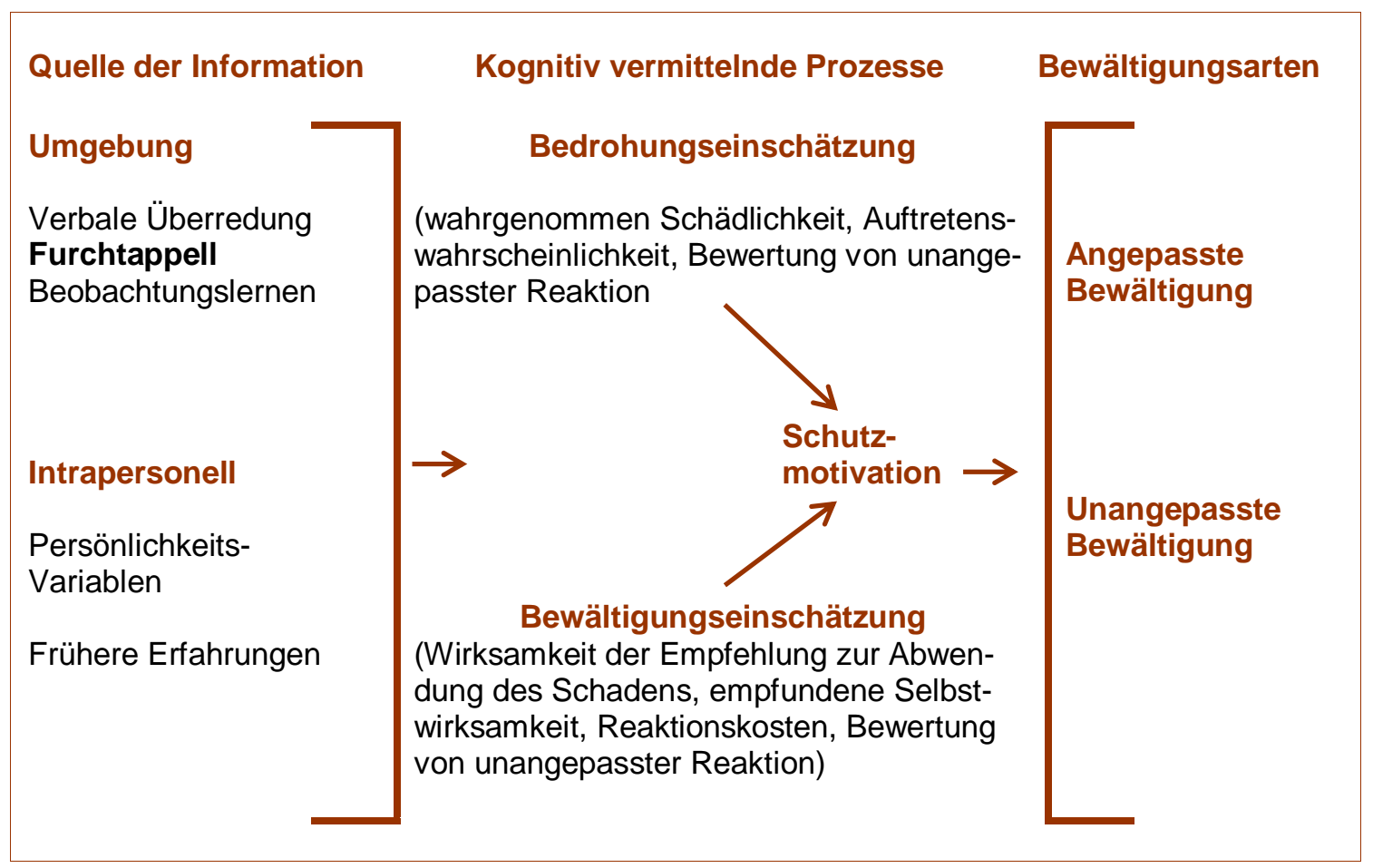

\footnotetext{
Abbildung 6: Revidierte Theorie der Schutzmotivation von Rogers 1983 ( modifiziert nach Rogers und Prentice-Dunn, 1997, S.114; übersetzt durch die Verfasserin)
}

Neben den bereits benannten Variablen spielen auch individuelle Unterschiede auf der Dimension Selbstwertgefühl (self-esteem) eine wichtige Rolle bei der Wirkung von Furchtappellen (Leventhal und Hirschman 1982).

Nach den Modell von Leventhal lassen sich zwei Prozesse von einander abgrenzen: Die Furcht- und die Gefahrenkontrolle. Es kann davon ausgegangen werden, dass die Furchtkontrolle (z.B. durch Anzweifeln der Information: „Ich glaube nicht dass Drogen wirklich schädlich sind“) nur kurzfristig Bedeutung hat, während langfristig die Gefahrenkontrolle (,Ich kann die Schäden infolge fortgesetzten Drogenkonsums abwenden, indem ich ein Behandlungsangebot wahrnehme, das mir hilft, drogenabstinent zu leben“) stärker motivierend wirkt.

\section{Weitere individuelle Einflussfaktoren}

Individuelle Faktoren wie Ängstlichkeit, Alter, Geschlecht, Ethnizität und Gruppenmitgliedschaft wurden ebenfalls hinsichtlich ihres Einflusses auf die persuasive Wirkung von Furchtappellen untersucht, wobei tendenziell kein Effekt auf die Ak- 
zeptanz der Empfehlung gefunden wurde (Witte und Allen 2000). Insgesamt sind die Untersuchungen zu individuellen Einflüssen jedoch noch zu wenig beweiskräftig (Sutton 1982; Witte und Allen 2000), als dass sich klare Vorhersagen über zielgruppenspezifische Furchtappellgestaltung machen ließen. Individuelle Einflüsse könnten aber zum geringen Konsens zwischen den vielen Furchtappelluntersuchungen beigetragen haben.

Weitere gezielte Untersuchungen zu zielgruppenspezifischer Furchtappellgestaltung auch im Bereich der Gesundheitsfürsorge sind daher dringend notwendig, um unterschiedliche Patientengruppen mit gezielten Botschaften zu erreichen und Verhaltensänderungen im Sinne der Gesundheitsprävention zu bewirken. Die vorliegende Arbeit soll dazu einen Beitrag leisten.

Im Unterschied zur vorliegenden Arbeit sind die meisten der benannten Untersuchungen unter Laborbedingungen durchgeführt worden, was die Validität der Befunde allgemein in Frage stellt.

\subsubsection{Der Furchtappell als Mittel zur Suchtprävention}

Furchtappelle werden zur Einstellungsänderung u.a. im Sinne der Beeinflussung des Gesundheits- und Vorsorgeverhaltens eingesetzt.

In der vorliegenden Untersuchung wird die Anwendung eines Furchtappells bei Personen mit einer stoffgebundenen Abhängigkeit untersucht. Es werden daher nun spezielle Gesichtspunkte für diese Personengruppe gesondert fokussiert.

Im Rahmen der Suchtprävention werden Furchtappelle und Warnhinweise zum Beispiel zur Bekämpfung des Alkoholismus, (MacKinnon et al. 1994; Stainback und Rogers 1983) oder der Nikotinabhängigkeit (Loken und Howard-Pitney 1988; Maddux und Rogers 1983; Sutton und Eiser 1984; Sutton und Hallet 1989; Watson et al. 1983) angewandt.

Exkurs zur Prävention:

Begriffsdefinition: Prävention ist ein sehr häufig gebrauchter Begriff, wobei eine allgemeingültige Definition fehlt. Als wichtige Unterteilung führte Caplan schon 1964 die Unterscheidung primärer, 
sekundärer und tertiärer Prävention ein. Ziel primärpräventiver Maßnahmen ist demnach die Senkung der Inzidenzrate von Erkrankungen. Die sekundäre Prävention bezeichnet die Frühdiagnostik auch unter Einsatz von Screeninguntersuchungen sowie die Frühtherapie von Krankheiten mit dem Ziel, einer Chronifizierung vorzubeugen. Der Begriff tertiäre Prävention deckt sich begrifflich weitgehend mit dem Konzept der Rehabilitation: Folgeschäden sollen vermieden oder beseitigt und eine berufliche Wiedereingliederung soll ermöglicht werden.

Eine weitere Klassifikation präventiver Maßnahmen stammt von Nasseri (1979): Unter Berücksichtigung der Zielgruppen einerseits und der zu erwartenden Effekte andererseits werden fünf Stufen der Prävention unterschieden: Nasseri differenziert zwischen (1) gesunden Personen, (2) gesunden Exponierten, (3) frühzeitig Erkrankten, (4) fortgeschritten Erkrankten und (5) chronisch Behinderten bzw. vom Tode bedrohten Personen. Als Prävention wird dabei jedes Verhindern des Absinkens auf eine tiefere Stufe definiert und ist somit für die unterschiedlichen Zielgruppen zu jeder Erkrankungsphase möglich. In diesem Sinne kann das in der vorliegenden Arbeit untersuchte Procedere auch als Studie zur Suchtprävention mittels eines Furchtappells gesehen werden.

Stoffgebundene Abhängigkeiten gehen mit drastisch verzerrten Einschätzungen der eigenen Gesundheitsgefährdung einher. Bzgl. Drogenabhängigkeit, Alkoholismus, Geschlechtskrankheiten, Suizid und Epilepsie wurden die größten Unterschätzungen des persönlichen Risikos für eine Gesundheitsgefährdung gefunden (Weinstein 1980,1984). Der Autor spricht dabei von einem unrealistischen Optimismus in Bezug auf die persönliche Gefährdung. Schwarzer $(1993,1994)$ baut auf diesem Konzept auf: Er unterscheidet zwischen einem funktionalen und einem defensiven Optimismus, wobei sich die defensive Form mit dem Konstrukt des unrealistischen Optimismus von Weinstein (1980) deckt und negativ mit dem Gesundheitsverhalten korreliert ist. Funktionale Optimisten hingegen überschätzen ihre Handlungsmöglichkeiten angesichts der Gefahren leicht und haben nach Schwarzer positive Korrelationen zu Kompetenz- und Handlungserwartungen, welche zu einer aktiven Problembewältigung beitragen.

Die beschriebene Unterschätzung des persönlichen Risikos für eine Gesundheitsgefährdung bei Drogenabhängigkeit führt möglicherweise dazu, dass die Suchterkrankung lange nicht als Problem von den Betroffenen gesehen wird. Daher werden oft über Jahre hinweg keine Strategien zur Problembewältigung entwickelt. Die Fehleinschätzung der Gefahren der Drogensucht macht eine Aufklärung und Korrektur nötig, sie könnte jedoch bedingen, dass besonders unpersönlich und allgemein gehaltene Furchtappelle am unrealistischen Optimismus der Betroffe- 
nen abprallen und nicht zu den gewünschten Einstellungs- und Verhaltensänderungen führen.

Betroffene Personen müssen demnach möglichst individuell angesprochen werden, sonst wird man sie kaum von der Notwendigkeit einer aktiven Problembewältigung (z.B. Entgiftungs- und Entwöhnungsbehandlung) überzeugen können. Diesen Aspekt gilt es besonders bei der Konzipierung eines Furchtappells für drogenabhängige Menschen - so auch in der vorliegenden Arbeit - zu berücksichtigen. 
Im Folgenden werden auf Basis der vorangegangenen Ausführungen die inhaltlich-klinischen Hypothesen bezüglich der Wirkung eines videobasierten Furchtappells im stationären Drogenentzug abgeleitet.

\subsubsection{Globale Fragestellung}

In der vorliegenden Arbeit

(1) soll festgestellt werden, ob mittels eines videobasierten Furchtappells das Durchhaltevermögen der betroffenen Patienten im stationären Drogenentzug nachhaltig beeinflusst werden kann.

(2) ist es auch das Ziel, die individuelle innere Reaktion der Rezipienten auf den Furchtappell zu berücksichtigen, um Wirkmechanismen genauer herausarbeiten zu können.

(3) gilt es, des Weiteren darzustellen, welche Bedeutung dem Geschlecht der Rezipienten für die Wirkung des Furchtappells zukommt.

\subsubsection{Klinische Erfahrungen aus dem Bereich der Entgif- tungsbehandlung}

Nach den klinischen Erfahrungen kommen viele Patienten immer wieder hoch motiviert zur Entgiftungsbehandlung und beteuern in der Aufnahmesituation ihren dringenden Entgiftungswunsch. Nicht selten befinden sie sich dabei in einem schlechten körperlichen und psychischen Gesundheitszustand: Sie sind oft abgemagert, haben Spritzenabszesse, sind ängstlich, intoxikiert oder entzügig. Daneben bedrücken sie zusätzlich häufig soziale (familiäre, finanzielle, behördliche...) oder forensische Probleme und chronische körperliche Erkrankungen. All dies wird 
oft schon in der Aufnahmesituation thematisiert und steht mehr oder weniger in Zusammenhang mit dem aktuellen Behandlungswunsch. Dennoch kommt es in ca. 50\% der Fälle zum Abbruch der Entgiftungsbehandlung (siehe auch Tabelle 1, S. 4), auch wenn die Opiatentgiftung methadongestützt durchgeführt wird (Gößling et al. 1998)! Dabei ereignen sich die meisten Behandlungsabbrüche in der Endphase der Entgiftung, wenn die Entzugssymptome am stärksten sind (Cook 1989). Die Patienten sind in dieser Phase schwer zu motivieren, die stationäre Therapie fortzusetzen. Verbalen Argumenten sind sie kaum noch zugänglich und alle bei der Aufnahme genannten motivationalen Faktoren und Behandlungsziele scheinen meist völlig in Vergessenheit geraten zu sein. Die im Falle eines Behandlungsabbruchs auftretenden Gefahren wie (1) Zunahme der Rückfallgefahr, (2) erhöhtes Sterberisiko, (3) Verschlimmerung der Suchterkrankung, (4) Zuspitzung sozialer oder forensischer Probleme werden genauso verleugnet wie die oft katastrophale soziale und gesundheitliche Situation, die zur Krankenhausaufnahme führte und dem Entgiftungswunsch zugrunde lag.

\subsubsection{Mögliche Bedeutung eines Furchtappells in der Entgif- tung}

\section{Zentrale Hypothese zur Abbruchquote}

Nach Untersuchungen von Leventhal und Watts (1966) ist ein direkter positiver Zusammenhang zwischen Ausmaß der Furchtinduktion und der Einstellungs- bzw. Verhaltensänderung zu erwarten.

Furchtinduktion sollte motivieren: Wichtig ist es daher zum Einen, dass Betroffene die Schädlichkeit des Suchtstoffgebrauchs erkennen und ihr persönliches Risiko für diesen Schaden (Vulnerabilität) adäquater einschätzen. Diese beiden wesentlichen Komponenten des Furchtappells sollen in der vorliegenden Untersuchung berücksichtigt werden. Die Patienten sollen ihren eigenen ursprünglichen Allgemeinzustand in einer abbruchgefährdeten Phase der Behandlung videobasiert gezeigt bekommen. Es ist zudem sehr wesentlich, neben der einfachen Furchtinduktion Bewältigungsmöglichkeiten zu vermitteln (Rogers 1983; Leventhal und 
Hirschmann 1982). Auch diesem Aspekt muss in der vorliegenden Untersuchung Rechnung getragen werden. In einem Nachgespräch im Anschluss an den Furchtappell müssen Bewältigungsmöglichkeiten aufgezeigt werden, um eingangs genannte Ziele zu erreichen.

Ein videobasierter Furchtappell sollte in der vorliegenden Studie auch helfen, in der Endphase der Entgiftung, wenn die Entzugssymptome am stärksten sind und die Abbruchgefahr am größten ist, die o.g. hartnäckigen Verleugnungen bezüglich der Rückfallgefahr und der Gesundheitsgefährdung durch die Suchterkrankung aufzulösen (vgl. unrealistischer Optimismus nach Weinstein 1980, 1984). Die Behandlungsmotivation soll gestärkt und insgesamt soll das Durchhaltevermögen im Rahmen der Entgiftungsbehandlung verbessert werden. Auch die „Kosten“ der Behandlung für den Patienten müssen daher berücksichtigt werden. Es erscheint günstig, mit dem Patienten möglichst weit reichende Strategien anzusprechen, die inm helfen, diese subjektiven Kosten zu reduzieren. Die empfohlenen Strategien müssten sehr konkret formuliert werden, so dass deren Umsetzung erleichtert wird. Die unterschiedlichen Möglichkeiten zum Ablenken von Entzugssymptomen erleichtern dem Individuum die Orientierung und fördern die Stärkung seines Selbstwirksamkeitsempfindens.

Unter Berücksichtigung der genannten Gesichtspunkte lautet die zentrale inhaltlich-klinischen Hypothese zur Wirkung eines videobasierten Furchtappells wie folgt:

KH-1:

Das Durchhaltevermögen im stationären Drogenentzug wird durch das Aufzeigen des ursprünglichen Allgemeinzustands (Furchtappell) bei gleichzeitigem Aufzeigen von Bewältigungsstrategien erhöht.

\section{Innere Verarbeitung}

Nach der klinischen Erfahrung handelt es sich besonders bei drogenabhängigen Menschen um eine Klientel, das ein spezielles, auf die spezifischen Bedürfnisse dieser Menschen abgestimmtes Vorgehen benötigt. Dies gilt u.a. sicher auch für 
Furchtappelle, um die Wahrscheinlichkeit zu maximieren, dass die persuasive Botschaft in der gewünschten Richtung verarbeitet wird. Die innere Informationsverarbeitung der Rezipienten hat sich wiederholt als entscheidender Faktor für die Wirkung eines Furchtappells erwiesen. Klinische Erfahrungen mit Suchtpatienten sprechen dafür, die Auslösung einer mittleren Furchtstärke anzustreben bei gleichzeitig ausreichender Vermittlung von Bewältigungsstrategien, um das Durchhaltevermögen im stationären Drogenentzug zu verbessern. Dieses würde den Annahmen von Rogers (1983) entsprechen. Daraus ergibt sich insgesamt eine weitere inhaltlich-klinische Hypothese:

$\mathrm{KH}-2$ :

Unter der Annahme, dass ein mittleres Maß an Furchtstärke nicht überschritten wird und Bewältigungsstrategien vermittelt werden, gilt: Die innere Verarbeitung des Furchtappells steht in Zusammenhang mit dem Durchhaltevermögen der Rezipienten - je mehr aversive emotionale Schemata aktiviert werden, um so eher wird das Durchhaltevermögen positiv beeinflusst.

\section{Induktion eines zusätzlichen Suchtdrucks}

Da erfahrungsgemäß der Suchtdruck und die Entzugssymptome am Ende einer Entgiftung (um den Nulltag herum) massiv sind, könnte zwar theoretisch auch noch zusätzlicher Suchtdruck durch eine Furchtinduktion erzeugt werden. Es wird jedoch wie bereits beschrieben ein therapeutisches Gespräch nach Ansicht des Videos geführt. In diesem Rahmen werden die bereits dargestellten Bewältigungsmöglichkeiten inklusive medikamentöser Strategien zur Dämpfung von Suchtdruck und Entzugssymptomen durchgegangen und angeboten. Eine weitere klinische Annahme lautet daher:

$\mathrm{KH}-3$ :

Der Furchtappell erzeugt keinen zusätzlichen Suchtdruck.

\section{Geschlechtsspezifische Wirkungen}

Es handelt sich bei drogenabhängigen Menschen um eine sehr heterogene Personengruppe (Geschlecht, Art des Suchtmittels, mono- oder polyvalenter Konsum, Krankheitsdauer...). Der Einfluss dieser interindividuellen Unterschiede auf die 
Verarbeitung eines Furchtappells bedarf an anderer Stelle einer ausführlichen Betrachtung. Ein einzelner Aspekt soll allerdings bereits in der vorliegenden Studie berücksichtigt werden: Geschlechtsspezifische Wirkungen bei der Verarbeitung des Furchtappells im Rahmen einer Entgiftungsbehandlung sind wahrscheinlich, da auch im Rahmen anderer gesundheitsbezogener Fragestellungen geschlechtsrelevante Unterschiede nachgewiesen werden konnten: Lairson und Swint (1978) zeigen, dass Frauen mittleren Alters höhere Punktwerte bezüglich des Stellenwerts der Gesundheit haben als gleichaltrige Männer. Außerdem fanden sie Hinweise darauf, dass bei Frauen im Vergleich zu Männern eine differenziertere, gleichzeitig umfassendere Sichtweise und eine intensivere Auseinandersetzung mit gesundheitsbezogenen Fragestellungen anzutreffen ist. Ferner sind Frauen präventiven Maßnahmen gegenüber positiver eingestellt und nehmen Angebote des medizinischen Gesundheitssystems, auch im Vorsorgebereich, mehr in Anspruch als Männer

Southwick-Bensley und Wu (1991) wiesen in einer Arbeit zur Einschränkung des Alkoholkonsums ebenfalls geschlechtsspezifische Unterschiede nach: Bei „mittleren bis schweren Trinkern“ erwiesen sich Männer nach einer dogmatischen Aufforderung zur Einschränkung des Alkoholkonsums als die Gruppe mit der größten Reaktanz und dem meisten Alkoholkonsum. Auch bei „leichten und mittleren Trinkern“ zeigten sich geschlechtsspezifische Unterschiede: Während Frauen bei einer Aufforderung zur Abstinenz mit einer geringeren Intention zu Trinken reagierten, zeigten Männer eine größere Trinkintention nach der Aufforderung zur Abstinenz.

Es ist davon auszugehen, dass sich auch in der vorliegenden Untersuchung bei drogenabhängigen Personen Reaktanzprozesse zeigen werden, die jedoch durch die individuelle Ansprache der Patienten etwas abgemildert werden können.

Es ergibt sich so die dritte inhaltlich-klinische Hypothese:

\section{KH-4:}

Die innere Verarbeitung eines Furchtappells weist geschlechtsspezifische Unterschiede auf: Frauen werden durch den videogestützten Furchtappell eher gefühlsmäßig berührt als Männer. 
Es werden nun der in dieser Studie angewandte Furchtappell sowie der Fragebogen, der zur Erfassung der inneren Verarbeitung dient, näher betrachtet. Anschließend wird das Untersuchungsdesign dargestellt, und die Gruppenbildung wird beschrieben. Nach Definition der Ein- und Ausschlusskriterien werden die medikamentösen und nichtmedikamentösen Behandlungsstrategien der stationären Entgiftung skizziert. Danach erfolgen die Operationalisierung der relevanten Merkmale, die Ableitung der statistischen Hypothesen sowie die Auswahl der notwendigen statistischen Verfahren. Abschließend wird die Untersuchungsstichprobe eingehend beschrieben und es wird geschildert, welche Personen letztlich ausgeschlossen wurden.

\subsection{Der Furchtappell}

Bei der Hälfte der teilnehmenden Patienten wurde das Aufnahmegespräch mit der Ärztin zu Beginn der Entgiftungsbehandlung mit einer Videokamera gefilmt. Intendiert war die Durchführung einer Videofeedbacksitzung am sog. „Nulltag“2 ${ }^{2}$ oder aber bei Abbruchwunsch der Patienten. Die Feedbacksitzung sollte in der Regel mit der Ärztin stattfinden. Bei Abbruchwunsch außerhalb der ärztlichen Dienstzeiten waren Mitglieder des Pflegeteams angeleitet worden, um die Videokonfrontation in einem solchen Fall durchführen zu können. Im Rahmen dieser Videokonfrontation sollte einerseits anknüpfend an die Aufnahmesituation audio-visuell mit den Gefahren für die Gesundheit und das Leben im Falle einer Drogenrückfälligkeit konfrontiert werden (Furchtappell) sowie andererseits an die bei Krankenhausaufnahme genannten Behandlungsmotivationen, Ressourcen und Therapieziele erinnert werden. Schon im Rahmen der Aufklärung über die Studie wurden die Rezipienten in diesem Sinne informiert und kognitiv vorbereitet. Der Nulltag wurde als Konfrontationstag für den videobasierten Furchtappell gewählt, weil einerseits

\footnotetext{
2 Nulltag heißt der erste Behandlungstag, an dem die zu entgiftende Substanz nicht mehr verabreicht wird. Werden mehrere Substanzen entgiftet, so zählt als Nulltag, der erste Tag an dem keine der zu entgiftenden Substanzklassen mehr eingenommen wurde
} 
nach den Erfahrungen des klinischen Alltags bei allen Patienten an diesem Tag starkes Suchtverlangen und Abbruchgedanken auftreten und andererseits, weil damit ein einheitlicher Punkt in den Entgiftungsverläufen festgelegt werden konnte, welcher deren Vergleichbarkeit ermöglicht.

Die Videodokumentation, das Vorgehen bei der Videokonfrontation und das Procedere bei Abbruchwunsch in der Kontrollgruppe werden nun im Detail beschrieben.

\subsubsection{Setting der Videodokumentation}

Das Arztzimmer, in dem das Aufnahmegespräch stattfand, welches mittels einer Videokamera auf Band aufgenommen wurde, ist bewusst eher gemütlich eingerichtet: Es soll den Patienten erleichtert werden, Vertrauen zu fassen, sich zu öffnen und über sich zu berichten. Die Videokamera ist, wenn man in der Eingangstür steht, etwa in der Mitte der linken Zimmerwand neben einer großen auf dem Boden stehenden Pflanze angebracht (siehe Abbildung 7, S. 52). Gegenüber der Eingangstür befindet sich ein Fenster mit pastellfarbenen Vorhängen und Blumen auf der Fensterbank. An der linken Wand, vor dem Fenster steht ein Schreibtisch mit einem drehbaren Schreibtischstuhl davor. Neben dem Fenster und dem Schreibtisch steht ein Bücherschrank. Er befindet sich also längs an der Wand mit dem Fenster, schräg gegenüber der Tür und reicht bis zum Ende des Zimmers an die rechte Zimmerwand. Gegenüber der Kamera bzw. neben dem Bücherschrank ist eine kleine Sitzgruppe aufgestellt, bestehend aus zwei Korbsesseln und einem runden Tisch. Ärztin und aufzunehmender Patient sitzen hier. Neben der Eingangstür steht direkt längs an der Wand, in der sich auch die Türöffnung befindet, eine Untersuchungsliege; diese steht also dem Bücherschrank gegenüber an der Wand. An den Wänden hängen mehrere größere und kleinere Bilder mit Landschaftsmotiven. Verbindet man die Standorte von Videokamera, Platz der Aufnahmeärztin und Platz der Patienten, so erhält man ein spitzwinkliges Dreieck.

Diese Dreieckkonstellation ist so gewählt, damit die Patienten im Gespräch mit der Ärztin sich nicht permanent in direkter Linie zur Kamera befinden. 


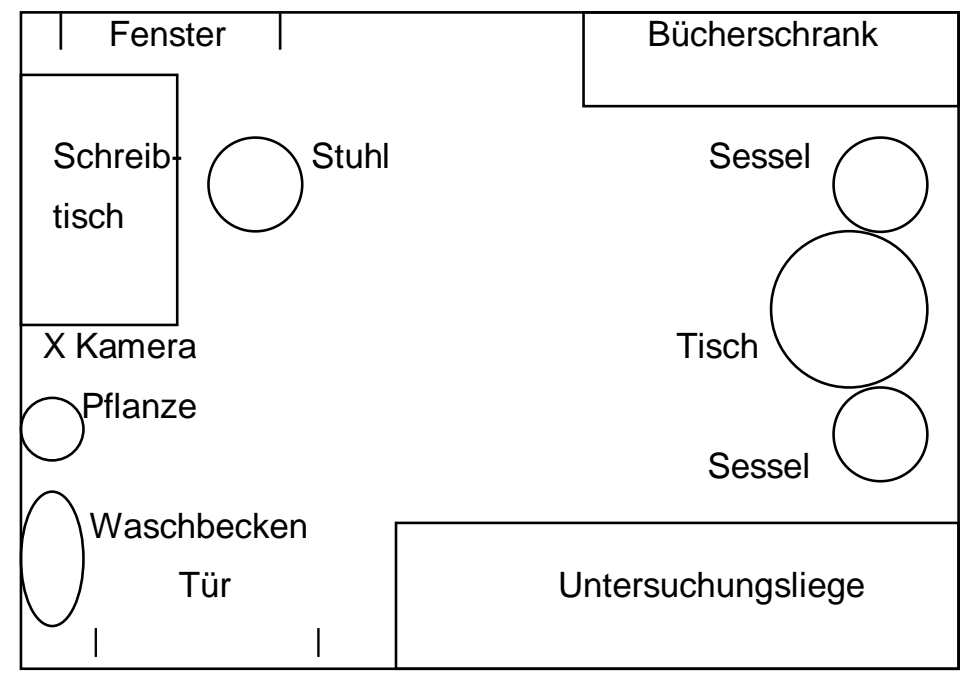

\section{Abbildung 7: Darstellung des Arztzimmers}

Wenn Patienten aufgrund ihrer Persönlichkeitsstruktur die Situation sehr zum Zwecke einer Selbstdarstellung zu nutzen versuchten, so wurde diesem Verhalten während der Aufnahme aktiv und steuernd von ärztlicher Seite entgegen gewirkt.

\subsubsection{Situation der Videokonfrontation}

Das Video vom Aufnahmetag sollte den Patienten jeweils am sog. „Nulltag“ gezeigt werden. In dieser Behandlungsphase sind erfahrungsgemäß die Entzugssymptome gekoppelt mit Suchtdruck und Abbruchtendenzen am stärksten. Die Patienten benötigen dann besondere Hilfe, um die Behandlung tatsächlich durchzuhalten.

Entstand ein Abbruchwunsch schon vor dem sog. Nulltag, sollte den entsprechenden Patienten ihr Video möglichst zu diesem Zeitpunkt gezeigt werden. Es wurde innen außerdem eine Reservemedikation (s.o.) zur Dämpfung von Suchtdruck und Entzugssymptomen angeboten. Es wurde im Einzelfall auch eine Reduktion der Entgiftungsgeschwindigkeit vorgeschlagen in der Hoffnung, die Patientin oder der Patient möge seinen Entschluss noch einmal überdenken und die Entgiftung trotz allem fortsetzen. 


\section{Phase der kognitiven Vorbereitung}

Das Video vom Aufnahmetag wurde bei der Konfrontation in der Regel zusammen mit der Ärztin angeschaut. Im Sinne einer kognitiven Vorbereitung wurden zuvor fokussierende Instruktionen gegeben: Die Patienten wurden darauf hingewiesen, dass es darum ginge, die Behandlungsmotivation in einer schwierigen Behandlungsphase zu stärken. In diesem Zusammenhang wäre auch das Video vom Aufnahmetag sehr wesentlich. Es sollte auch an die bei der Krankenhausaufnahme genannten Behandlungsziele erinnert und mit den Gefahren im Falle einer Drogenrückfälligkeit konfrontiert werden. Die einleitenden Instruktionen waren bewusst gewählt, denn es ist erwiesen, dass eine kognitive Vorbereitung den therapeutischen Effekt des Videofeedbacks erhöht (Kim et al. 2002).

\section{Eigentliche Phase der Videokonfrontation}

Die Videokonfrontation wurde in einem Aufenthaltsraum für das Personal der Station durchgeführt, da hier ein Fernsehgerät und ein Videorekorder vorhanden sind. In dem Raum befinden sich außerdem eine Sitzgruppe mit einem runden Tisch, einigen Stühlen und einem Ecksofa, des Weiteren ein kleiner Schrank, ein Kühlschrank und eine Spüle. Zum Zwecke der Videokonfrontation wurde der Raum der Ärztin und dem Patienten überlassen. Den Patienten war es erlaubt, während des Videoplaybacks frei von sich heraus zu kommentieren. Bei längeren Kommentaren (definiert als „länger als ein Satz") wurde das Band angehalten. Die Therapeutin war zurückhaltend, während das Band lief, zeigte sich jedoch bei Gesprächsbedarf der Rezipienten resonant, empathisch, Ressourcen fördernd und die Selbstwirksamkeit stärkend. Diese Unterstützung hinsichtlich Selbstwirksamkeitsempfinden und Selbstwertgefühl sind nach Rogers $(1975,1983)$ sowie Leventhal und Hirschmann (1982) zentrale Aspekte, die für eine ausreichende Wirkung eines Furchtappells notwendig sind. Bilodeau und Bilodeau kamen $1969 \mathrm{zu}$ dem Schluss, dass das Feedback sofort und nicht verzögert erfolgen sollte, um den Effekt zu maximieren. Ausgehend von diesen Schlussfolgerungen wurde entschieden, ein laufendes Replay zu unterbrechen, um sofort Kommentare zu geben (Griffiths 1974). 
Direkt im Anschluss an das Video erhielten die Patienten einen Fragebogen, welcher die individuelle Reaktion auf das Video erfasste (siehe Kapitel 2.2. Fragebogen zur Erfassung der inneren Verarbeitung; S. 57)

\section{Die Phase der Nachbereitung}

Die Videokonfrontation wurde dann in einem sofort anschließenden therapeutischen Gespräch mit den Patienten nachbereitet. Dabei wurde einerseits auf die Äußerungen der Probanden eingegangen. Es bestand z.B. die Möglichkeit, übermäßig heftige Schamreaktionen zu bearbeiten oder bei zu geringer affektiver Beteiligung abgewehrte Affekte erlebbar zu machen. Es wurde angestrebt, auf diese Weise ein mittleres Ausmaß aversiver emotionaler Aktivierung zu erreichen. Andererseits wurde auch detailliertes Feedback durch die Ärztin gegeben. Intendiert war, den Verleugnungen der Gefahren der Suchterkrankung entgegen zu wirken und den unrealistischen Optimismus zu schmälern. Eine direkte Orientierung am Einzelfall ist hier sehr wichtig, damit die Betroffenen sich tatsächlich persönlich angesprochen fühlen.

Es wurde von ärztlicher Seite aktiv auf die im Film sichtbaren schädlichen Drogenwirkungen hingewiesen. Auch wurden vitale und soziale Gefahren im Falle des Behandlungsabbruchs und der Drogenrückfälligkeit erörtert. Außerdem wurden die bei Aufnahme geäußerten Behandlungsziele erneut ins Bewusstsein gehoben und Bewältigungsmöglichkeiten der aktuellen Krise aufgezeigt: Es wurden Strategien besprochen, welche helfen sollten, die Behandlung durchzuhalten. So wurde darauf hingewiesen, dass bei starker vegetativer Entzugssymptomatik und Suchtdruck die o.g. Reservemedikation zur Verfügung steht; die individuellen „Kosten“ bei der Fortsetzung der Entgiftungsbehandlung werden so minimiert. Es wurde erläutert, dass Entzugssymptome und Suchtdruck wie andere Gefühle langsam zunehmen, ein Maximum erreichen und dann auch wieder nachlassen. Die Probanden wurden darüber aufgeklärt, dass es beim Auftreten von Entzugssymptomen und Suchtdruck hilfreich sein kann, den Fokus der Aufmerksamkeit nach auBen zu richten, um den Entgiftungsstress so zu überbrücken. Dazu wurden Möglichkeiten der Ablenkung wie sportliche Aktivitäten, Gespräche, Ergotherapie, 
Fernsehen und Musikhören angeboten. Die Selbstwirksamkeit wurde gestärkt, z. B. durch Erinnern an erfolgreiche Entgiftungen in der Vergangenheit.

Nachgewiesen wurde mehrfach, dass ein Videofeedback allein nur von geringem Nutzen ist, während bei einer Kopplung von Videofeedback und therapeutischen Instruktionen zu Bewältigungsstrategien positive therapeutische Effekte nachgewiesen werden konnten (Baker et al. 1975; Eisler et al. 1973; Harvey et al. 2000; Kim et al. 2002; Rapee und Hayman 1996). Detailliertes Feedback scheint effektiver zu sein als Feedback in einer generalisierten Form (wie z.B.: „richtig“ oder „falsch“ McGuigan 1959). Daher wurden auch diese beiden Aspekte bei der Planung des Vorgehens berücksichtigt.

\subsubsection{Procedere bei Abbruchwunsch von Kontrollpersonen}

Wollte eine Person der Kontrollgruppe die Behandlung abbrechen, so erhielt diese Person ein eingehendes Gespräch mit der Ärztin. Dieses entsprach nach Form und Inhalt dem Gespräch, das die Rezipienten der Experimentalgruppe bei Abbruchwunsch erhielten. Nach den Beweggründen für die vorzeitige Beendigung der Therapie wurde gefragt. Auf die Gefahren, die bei einem Behandlungsabbruch drohen, wie erhöhte Rückfallgefahr, Verschlimmerung der Suchterkrankung, negatives Selbsterleben, Überdosierung von Drogen etc. wurde hingewiesen. Es wurde an die bei Krankenhausaufnahme genannten Behandlungsziele erinnert, um doch noch zur Fortsetzung der Entgiftungsbehandlung zu motivieren. Des Weiteren wurden auch den Abbrechern in der Kontrollgruppe konkrete Bewältigungsmöglichkeiten der aktuellen Krise aufgezeigt (Einnahme einer Reservemedikation, Hilfe durch Gespräche mit Personal, Ablenkung durch Ergotherapie, Sport, Fernsehen, Musik, Stärkung der Selbstwirksamkeit z. B. durch Erinnern an erfolgreiche Entgiftungen in der Vergangenheit, es wurde hingewiesen auf den Rückgang von Suchtdruck und Entzugssymptomen im Laufe der Zeit). 


\section{$2.2 \quad$ Fragebogen zur Erfassung der inneren Verarbeitung}

Es wurde bereits darauf hingewiesen, dass die Variable der inneren Verarbeitung mittels eines selbstkonstruierten Fragebogens operationalisiert wurde. Aufgrund der Zielgruppenspezifität der Furchtappelle gab es keinerlei bewährte Instrumente, auf die an dieser Stelle hätte zurückgegriffen werden können. Um relevante Items zu finden, wurden daher einige Patienten im Rahmen einer kurzen Pilotphase im Vorfeld der aktuellen Studie zu ihren Reaktionen auf den videobasierten Furchtappell befragt. Geäußert wurden positive und negative Reaktionen auf das Videofeedback wie: Das Video deprimiert mich (bzw. deprimiert mich nicht), hilft mir, die Behandlung durchzuhalten, (hilft mir nicht, die Behandlung durchzuhalten). Angelehnt an die hauptsächlich genannten emotionalen, kognitiven und handlungsbezogenen Reaktionen wurde der in der Studie angewandte dreistufige Fragebogen entworfen, den die Rezipienten direkt im Anschluss an die Videokonfrontation ausfüllen sollten, bevor dann das therapeutische Gespräch stattfand (siehe Abbildung 8, Seite 57).

Darüber hinaus interessierte die Frage, ob die Videokonfrontation einen negativen bzw. schädlichen Einfluss auf den Verlauf der Entgiftungsbehandlung durch Verstärkung des Suchtdrucks hat, daher wurde zu diesem Gesichtspunkt ein weiteres Item formuliert.

Um zu erfahren, wie die Zielgruppe selbst den Nutzen der Videokonfrontation beurteilt, wurde ein weiteres Item ergänzt: Es wurde danach gefragt, ob die Videokonfrontation als grundsätzlicher Bestandteil des therapeutischen Vorgehens in den Therapieplan aufgenommen werden sollte. Die Beantwortung erfolgte hier kategoriell mit den Möglichkeiten „Ja“ und „Nein“. 


\section{Code:}

1. Das Video berührt mich gefühlsmäßig.

2. Es ist mir gleichgültig.

nicht etwas sehr

3. Es ist mir peinlich.

nicht etwas sehr

4. Es macht mir Suchtdruck.

nicht etwas sehr

5. Es stößt mich ab.

nicht etwas sehr

6. Ich finde es hilfreich, um die Entgifnicht etwas sehr tung durchzuhalten.

nicht etwas sehr

7. Es deprimiert mich.

nicht etwas sehr

8. Es hat für mich keine Bedeutung. Diese Aussage stimmt. nicht etwas sehr

Ich würde es bei allen Patienten machen: Ja / nein

\section{Abbildung 8: Fragebogen nach Ansicht des Videos}

Es ist davon auszugehen, dass eine faktorielle Prüfung der acht zentralen Items dieses Fragebogens zu zwei Faktoren führen wird:

Skala / Faktor 1: Gefühlsmäßige Berührung

Skala / Faktor 2: Suchtdruck

\subsection{Darstellung des Untersuchungsdesigns}

Für die Entscheidung darüber, ob das Aufzeigen des ursprünglichen Allgemeinzustands (zum Zeitpunkt des Entzugsbeginns) mittels einer Videodokumentation geeignet ist, um das Durchhaltevermögen nachhaltig zu beeinflussen, wurde ein Zwei-Gruppendesign gewählt: Eine Gruppe von Patienten erhielt eine Videodokumentation ihres Allgemeinzustands am ersten stationären Behandlungstag und 
sollte das entsprechende Video am sogenannten „Nulltag“ gezeigt bekommen. Im Falle eines drohenden Behandlungsabbruchs vor dem Nulltag sollten die Probanden das Video schon dann gezeigt bekommen. Das Video wurde zusammen mit der Ärztin angeschaut und wie bereits an anderer Stelle beschrieben in einem direkt anschließenden therapeutischen Gespräch mit den Patienten nachbereitet. Die Kontrollgruppe erhielt weder die Videoaufzeichnung noch die Videokonfrontation am Nulltag bzw. bei Äußerung des Abbruchwillens. In diesem Fall erfolgte lediglich ein therapeutisches Gespräch.

\subsection{Darstellung der experimentellen Variation/ Gruppenbil- dung}

Untersucht wurden Patienten beiderlei Geschlechts, welche zwischen Mai 2004 und Dezember 2005 zur stationären Entgiftung illegaler Drogen im ehemaligen Niedersächsischen Landeskrankenhaus Göttingen auf einer Drogenentzugsstation aufgenommen worden waren. Das Niedersächsische Landeskrankenhaus Göttingen war ein psychiatrisches Krankenhaus der Regionalpflichtversorgung für den Bereich Süd-Niedersachsen.

Der Untersuchung wurde eine randomisierte Stichprobe von insgesamt 120 Patienten zugrunde gelegt. Es ist bekannt, dass sich das Geschlechterverhältnis Frauen / Männer in der Suchttherapie illegal drogenabhängiger Patienten auf 1 / 3 bzw. 1 / 4 beläuft (siehe Tabelle 1, Seite 4). In der vorliegenden Studie wurde die Stichprobe dem entsprechend geschichtet randomisiert und bestand aus je 20 Frauen und je 40 Männern in der Experimental- bzw. Kontrollgruppe. Die Zuordnung der Patienten in die Gruppe derer, die videografiert wurden bzw. in die Vergleichsgruppe, bei der keine Videoaufnahme gemacht wurde, erfolgte unmittelbar im Anschluss an die Einwilligung in die Studienteilnahme nach einem Randomisierungsplan. Beide Gruppen waren auf einer Station untergebracht und wussten voneinander. 


\subsubsection{Ablauf}

Die Studie begann mit der stationären Aufnahme.

Die Patienten wurden sofort (innerhalb der ersten Stunde nach der Krankenhausaufnahme) über die Studie aufgeklärt und erhielten Aufklärungs- und Einwilligungsformulare. Eingeschlossene Patienten (nach Prüfung auf Ein- und Ausschlusskriterien - s. u.) galten als in die Studie aufgenommen, wenn sie sich schriftlich hierzu einverstanden erklärt hatten und das halbstandardisierte Aufnahmegespräch ${ }^{3}$ hinter sich gebracht hatten.

Zur Auswahl kamen Patienten mit einer Opioidabhängigkeit bzw. Patienten mit einer Polytoxikomanie inklusive einer Opioidabhängigkeit entsprechend den Kriterien der ICD-10 (Dilling et al. 2000). Hinsichtlich der Suchterkrankungen wurden, sofern die Diagnosekriterien für eine Opioidabhängigkeit nach ICD-10 erfüllt waren, auch Mehrfachdiagnosen gestellt. Die stationäre Aufnahme der Patienten erfolgte zur stationären Entgiftung von Opioiden oder zur Beikonsumentgiftung unter laufender Substitutionstherapie mit Levomethadon bzw. Buprenorphin bei einer Vorgeschichte mit Konsum illegaler Drogen. Auch eingeschlossen wurden Fälle, die neben illegalen Drogensucht vom Opioidtyp suchterzeugende Arzneimittel und/oder Alkohol aktuell konsumiert hatten.

Folgende Einschlusskriterien bestanden:

1. Die Diagnose einer Opioidabhängigkeit (ICD-10: F 11.2) und fakultativ auch polyvalenter Substanzmissbrauch inklusive Opioidabhängigkeit (ICD-10: F 19.2) entsprechend den Kriterien der ICD-10 musste vorliegen.

2. Die Patienten hatten als Therapieziel einen Entgiftungswunsch und

3. waren mindestens 18 Jahre, höchstens aber 65 Jahre alt,

4. geschäftsfähig und mit der Teilnahme an der Studie einverstanden.

5. Sie unterlagen keiner gesetzlichen Betreuung.

\footnotetext{
${ }^{3}$ Die Befunderhebung bei Aufnahme war angelehnt an die Dokumentation Standards 3 für Evaluation der Behandlung von Abhängigen (Deutsche Gesellschaft für Suchtforschung und Therapie e.v. 2001).
} 
6. Die stationäre Aufnahme erfolgte direkt auf der Suchtstation und nicht als Verlegung innerhalb der Klinik.

Bei den Personen, die die Einschlusskriterien erfüllten, wurden des Weiteren die folgenden Ausschlusskriterien geprüft:

1. die gleichzeitige Teilnahme an einer anderen klinischen Prüfung,

2. das Vorliegen von Schwangerschaft oder Stillperiode,

3. eine akute Psychose (einschließlich eines akuten Erregungszustands),

4. das Fehlen oder die Rücknahme des Einverständnisses der PatientIn,

5. der Zugang gemäß Psychischkrankengesetz Niedersachsen (Zwangseinweisung),

6. die Unterbringung als erkrankter Häftling, sowie

7. ein bereits bestehendes schweres Entzugssyndrom.

8. Ebenfalls ausgeschlossen werden mussten Patienten, die keinerlei Begleitmedikation im Rahmen der Entgiftungsbehandlung benötigten (bei innen war kein Nulltag vorhanden!).

\subsection{Darstellung des multimodalen Therapiekonzepts auf der Entgiftungsstation}

Im stationären Drogenentzug wurden im Rahmen eines multimodalen Therapiekonzepts entsprechend den aktuellen medizinischen Standards unterschiedliche medikamentöse und nicht-medikamentöse Begleittherapien eingesetzt. Diese werden im Folgenden dargestellt:

\subsubsection{Medikamentöse Entgiftungsschemata:}

\section{a) Opioidentgiftung:}

Opioidentgiftungen wurden mit Levomethadon (L-Polamidon oder Methadon 1\%) und/oder Buprenorphin durchgeführt. Nach einer Phase der Dosisfindung und Einstellung von zwei Tagen erfolgte die Abdosierung ab dem dritten Behandlungstag. 
Bei Dosen jenseits von $5 \mathrm{ml} /$ Tag wurde in der Regel um $1 \mathrm{ml} /$ Tag reduziert, ab $5 \mathrm{ml}$ erfolgte tägliche Abdosierung um $0,5 \mathrm{ml}$ pro Tag. Bei einer Tagesdosis von 2 $\mathrm{ml}$ täglich bestand die Möglichkeit einer Fortsetzung der Opioidentgiftung mittels Buprenorphin. Bei Opiatabhängigkeit mit täglicher Heroineinnahme bis zu maximal $3 \mathrm{~g}$ täglich bestand auch die Möglichkeit einer unmittelbaren Einstellung auf Buprenorphin zum Zwecke der Entgiftung. Zur Dämpfung vegetativer Entzugssymptome erhielten die Patienten ab einer täglichen Methadondosis von $5 \mathrm{ml}$ oder weniger bei Bedarf bis maximal $300 \mathrm{mg}$ Doxepin oder Trimipramin pro Tag, bis $600 \mu \mathrm{g}$ Catapresan pro Tag, ein niederpotentes Neuroleptikum zur Nacht, wenn über Schlafstörungen geklagt wurde.

\section{b) Kokainentgiftung:}

Eine Kokainentgiftung wurde Benzodiazepin gestützt durchgeführt. Nach einer Phase der Dosisfindung von zwei Tagen erfolgte die schrittweise Abdosierung ab dem dritten Behandlungstag. Zur Stabilisierung der Stimmungslage wurde in der Regel ein Antidepressivum vom Typ der Serotoninreuptakehemmer verabreicht. Bei Schlafstörungen wurde ein niederpotentes Neuroleptikum zusätzlich verordnet.

\section{c) Benzodiazepinentgiftung:}

Die Benzodiazepinentgiftung wurde mittels Diazepam oder Clonazepam unter Anfallsschutz mit Carbamazepin durchgeführt. Nach einem 2-3-tägigen Intervall der Dosisfindung wurde die Abdosierung schrittweise (täglich ca. 10\% der Ausgangsdosis weniger) vorgenommen.

\section{d) Alkoholentgiftung:}

Im Rahmen der Alkoholentgiftung wurde zur Dämpfung vegetativer Entzugssymptome Clomethiazol, Diazepam oder Clonazepam verordnet, wobei die Abdosierung schrittweise nach einer initialen 2-tägigen Phase der Dosisfindung erfolgte. Zum Anfallsschutz wurde begleitend Carbamazepin verordnet. 


\section{e) Cannabisentgiftung:}

Die Cannabisentgiftung erfolgte in der Regel ohne eine spezielle Begleitmedikation, nur durch Einhaltung von Abstinenz.

\section{f) Entgiftung mehrerer Suchtstoffe:}

Bei Detoxifikation mehrerer Suchtstoffe kamen die o.g. Entgiftungsschemata zur Anwendung. Die Entgiftungen wurden überlappend durchgeführt, jedoch in der Regel so terminiert, dass nicht für alle Substanzen im selben Moment der Nulltag erreicht wurde, um den Entgiftungsstress in Grenzen zu halten. In diesem Falle wurde zur Auswertung der Frage ,Abbruch ja/nein' der letzte Nulltag als Ausgangspunkt für die Studie genommen.

Unzulässig war die selbstständige Einnahme von Medikamenten (einschließlich rezeptfreier Medikamente), die nicht durch die Stationsärztin verordnet worden sind.

Bei entsprechender medizinischer Indikation wurde eine spezielle Diät ärztlich verordnet. Alle Patienten durften keine mohnhaltigen und keine alkoholhaltigen Nahrungsmittel zu sich nehmen. Die Probanden erhielten das im NLKH Göttingen übliche Patientenessen.

\subsubsection{Sonstige Begleittherapien}

Die Mitbehandlung somatischer und psychiatrischer Erkrankungen wurde entsprechend den Regeln der Kunst (einschließlich aller Psycho- und Pharmakotherapien) durchgeführt. Eine Interferontherapie bei Hepatitis-C-Virusinfektion wurde im Untersuchungszeitraum bei keinem der Teilnehmer gemacht. Die Behandlung wurde von der zuständigen Oberärztin überwacht. Sport- und Physiotherapie fanden mehrfach wöchentlich statt. Das diesbezügliche Angebot umfasste Konditionstraining, Krafttraining, Spaziergänge, Joggen, Kegeln, Bowling, Walken, Gymnastik, Rückengymnastik, Übung zur Entspannung, Bäder, Fango-Packungen und Massagen. 
Im Rahmen der Ergotherapie gab es die Möglichkeit, an handwerklichkonstruktiven sowie kreativ-gestalterischen Arbeiten teilzunehmen. Neben den umfangreichen Aufgaben der alltäglichen Routine war pflegerischer Schwerpunkt die Kontakt- und Beziehungsaufnahme zu den Patienten. In der täglichen Morgenrunde wurden anstehende Termine, Tagesabläufe und Schwierigkeiten auf Station besprochen. Beratung durch einen Sozialarbeiter erfolgte individuell nach Bedarf. Ärztliche Visiten oder Kurzvisiten fanden werktäglich statt. Jeder Patient der Kontrollgruppe erhielt am Nulltag ein ärztliches Gespräch, um Hilfsmöglichkeiten bei Suchtdruck und Abbruchtendenzen aufzuzeigen und die Behandlungsmotivation zu stärken. Darüber hinaus wurden Einzelgespräche auf stützender und kognitivverhaltenstherapeutischer Grundlage nach Bedarf durchgeführt. Akupunktur zur Entzugserleichterung erhielten Patienten, wenn sie es wünschten. Gruppentherapeutische Angebote waren teils fakultativ (Entspannungsgruppe), teils Pflichtbestandteil des Behandlungsprogramms (psychoedukative „Entgiftungsgruppe“).

\subsection{Datenerhebung}

Primäre Zielgröße ist der Therapieabbruch, d.h. der Patient kündigt das Arbeitsbündnis auf und bricht die Entgiftung vorzeitig ab. Als abgeschlossen gilt eine Entgiftungsbehandlung in der vorliegenden Studie, wenn sie bis zum 5. Nulltag durchgehalten wurde. Gemessen werden sollte der Einfluss der Videodokumentation und -konfrontation (einschließlich der Nachbereitung) auf das Durchhaltevermögen bzw. das Abbruchverhalten der Probanden. Die Videoaufnahme erfolgte am ersten stationären Behandlungstag.

Die soziodemographischen Daten wurden bei Aufnahme im Rahmen eines halbstandardisierten Interviews erhoben. Die Erfassung der erhobenen Befunde erfolgte gemäß Prüfplan mittels Erhebungsbögen (siehe Anhang C, Seite 124 f.) sowie Videoaufzeichnung. Folgende Daten wurden erfragt: Alter Geschlecht, Familienstand, berufliche Situation, Einkommen, Wohnsituation, psychische und körperliche Krankheiten, Suizidversuche, Suizidalität, Suchtanamnese einschließlich Familienanamnese, somatische Anamnese, psychiatrische Anamnese, abstinenzerhaltende Maßnahmen nach dem Entzug, z.B. Naltrexonbehandlung oder Langzei- 
tentwöhnungsbehandlung, Biografie, schulischer und beruflicher Werdegang, traumatische Erfahrungen, forensische Situation, Therapieziele, Perspektiven, Therapiemotivation, therapeutische Vorerfahrungen und die Wartezeit bis zur stationären Aufnahme. Es wurde eine internistische und neurologische klinische Untersuchung durchgeführt, der psychische Befund wurde ebenfalls erhoben. Folgende Laborparameter wurden darüber hinaus bestimmt: Regelmäßig wurden Suchtstoffscreenings im Urin durchgeführt, erstmals am Aufnahmetag und dann mindestens 3x/Woche. Bei jedem Patienten wurden folgende Blutuntersuchungen durchgeführt: Blutsenkungsgeschwindigkeit, Blutbild, Elektrolyte, Lebertransaminasen, Creatin-Kinase, Blutzucker, Blutfette, Albumin, Thyroidea-stimulierende Hormone, Gerinnungswerte, harnpflichtige Substanzen, Hepatitis-Serologie (Hepatitis A-, B- und C Antikörper), HIV-Test, wenn gewünscht. Bei den Frauen wurde regelhaft ein Schwangerschaftstest (Beta-HCG im Urin) durchgeführt.

Die anamnestischen Daten bzw. die Compliance wurden kontrolliert über Vergleiche der aktuell ärztlich erhobenen Befunde mit der Pflegeanamnese und den Vorbefunden aus den in der Klinik befindlichen Akten oder auch aus Berichten auswärtiger Kliniken. Von letzteren wurden Befunde nach schriftlichem Einverständnis der jeweiligen Patienten angefordert. 


\subsubsection{Variablen und angenommene Wirkungszusammen- hänge im Überblick}

Es wurden bereits verschiedene relevante Variablen benannt, deren Wirkzusammenhänge nun im Überblick betrachtet werden sollen. Die experimentelle Variation des Furchtappells ermöglicht es, Hinweise auf einen eventuell bestehenden kausalen Einfluss dieser Variable für das Durchhaltevermögen der Probanden nachzuweisen. Die innere Verarbeitung der Rezipienten sowie das Geschlecht stellen quasiexperimentelle Variablen dar. Aufgrund dieses sonst quasiexperimentellen bzw. korrelativen Designs ist es bezüglich dieser Variablen nicht möglich, kausale Beziehungen zwischen den einzelnen Merkmalen nachzuweisen. Es kann jedoch überprüft werden, ob Kovariationen zwischen den Merkmalsausprägungen zu finden sind, um nähere Hinweise auf die zugrunde liegenden Wirkmechanismen zu erhalten (siehe Abbildung 9, S. 65; Kovariationen sind durch Linien dargestellt, während der mögliche Kausalzusammenhang als Pfeil repräsentiert ist).

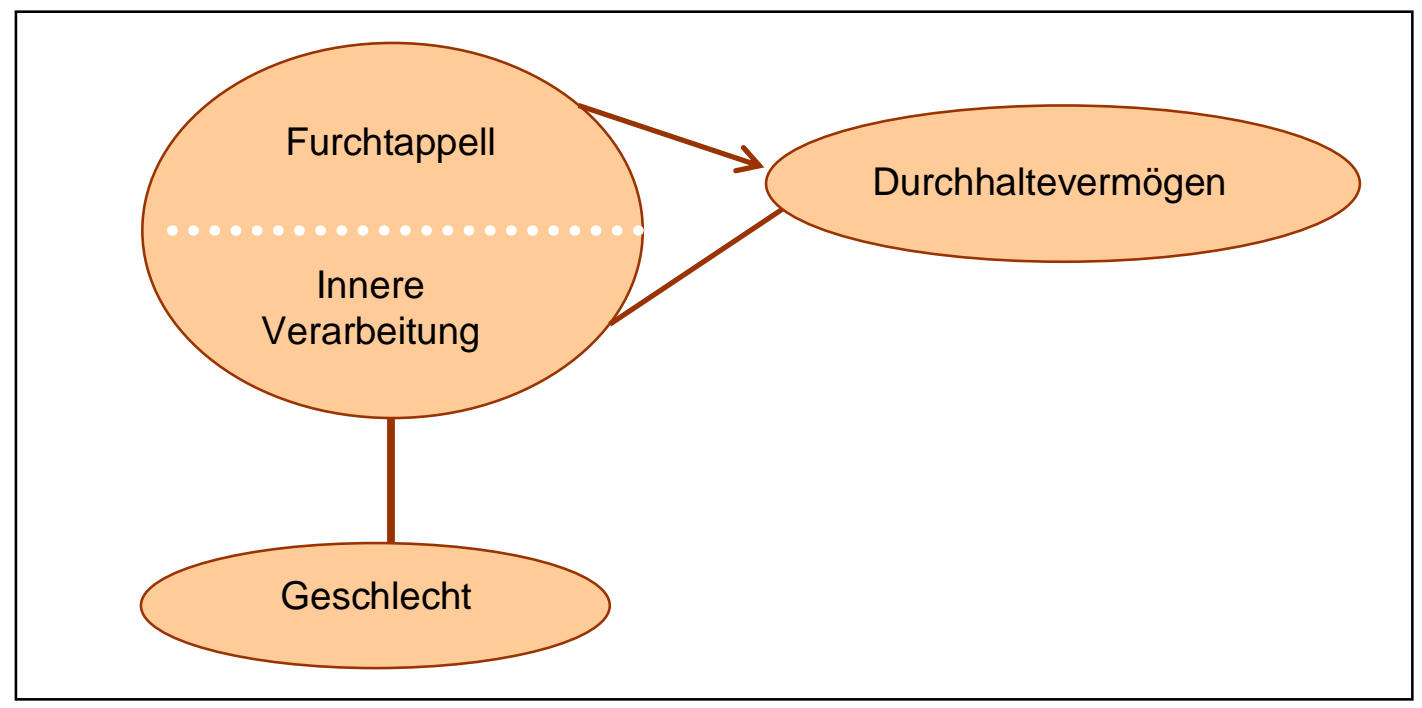

Abbildung 9: Darstellung der untersuchten Variablen bzw. Merkmale mit den jeweils in der vorliegenden Studie betrachteten (Wirkungs-) Zusammenhängen 


\subsubsection{Zuordnung der Variablen und Operationalisierung}

Die Unabhängige Variable in dieser Untersuchung ist die Durchführung der Videodokumentation bzw. auch -konfrontation.

Das Durchhaltevermögen im stationären Entzug stellt die abhängige Variable dar. Es wird hinreichend operationalisiert, indem ein eventueller Abbruch des stationären Aufenthalts wie beschrieben erfasst wird.

Die innere Verarbeitung des in der vorliegenden Studie eingesetzten videobasierten Furchtappells und das Geschlecht der Patienten wurden als weitere relevante Merkmale innerhalb der Gruppe derer, die das Video sahen, ebenfalls erhoben. Die innere Verarbeitung wurde durch den Fragebogen erfasst, der mittels einer dreistufigen Ratingskala beantwortet wurde. Es wurden (wie bereits beschrieben) kognitive, affektive und handlungsbezogene Reaktionen der Rezipienten auf den Furchtappell erfragt.

Es war zudem natürlich sehr wichtig, zu erfassen, ob die Personen mit einer Videodokumentation das Video auch tatsächlich angesehen haben, d.h. einer Videokonfrontation letztlich zustimmten. Die Experimentalgruppe lässt sich mittels dieser Variablen in zwei Subgruppen aufteilen. In Abbildung 10 (S. 66) wurden die Gruppen im Überblick abgebildet:

\begin{tabular}{|l|l|l|}
\hline \multicolumn{2}{|l|}{ UV Video } & Experimentalgruppe \\
\hline Kontrollgruppe & Vdoku & Vdoku+Vkonf \\
\hline Keine Vdoku+Vkonf & (Gruppe 2a) & \\
(Gruppe 1) & & (Gruppe 2b) \\
\hline
\end{tabular}

Abbildung 10: Versuchsplan - Aufteilung der Gruppen im Überblick 


\subsubsection{Ableitung der klinischen Vorhersagen und statisti- schen Hypothesen}

Ausgehend von der vorgenommen Operationalisierung ist es nun möglich, den inhaltlich-klinischen Hypothesen klinische Vorhersagen zuzuweisen. Diese Vorhersagen stellen den Bezug zum Versuchsplan her. Die statistischen Hypothesen, die diesen Vorhersagen zugeordnet werden, übertragen die Annahmen in statistische Kennwerte. Aus diesen ergeben sich schließlich die geeigneten statistischen Verfahren, die als Hilfsmittel zur Überprüfung der inhaltlichen Hypothesen zu betrachten sind.

In Kapitel 1.6 (Seite 45 f.) wurden die inhaltlich-klinischen Hypothesen bereits umfassend hergeleitet.

Zentrale Hypothese zur Abbruchquote $(\mathrm{KH}-1)$

KH-1:

Das Durchhaltevermögen im stationären Drogenentzug wird durch das Aufzeigen des ursprünglichen Allgemeinzustands (Furchtappell) bei gleichzeitigem Aufzeigen von Bewältigungsstrategien erhöht.

Im Sinne daraus abzuleitender klinischer Vorhersagen bedeutet dies:

KV-1:

Die Häufigkeit von Abbrüchen des Entzugs ist bei den Patienten, die einen Furchtappell gezeigt bekommen, geringer als bei denjenigen, die kein entsprechendes Video ansehen.

Kann die klinische Vorhersage empirisch belegt werden, so gilt die klinische Hypothese als bestätigt. Die Abbildung auf die statistische Ebene muss erschöpfend erfolgen.

$\mathrm{KH}-1$ wird auf statistischer Ebene abgebildet als eine Hypothese bezüglich Häufigkeitsangaben. Die zugehörige statistische Hypothese sieht wie folgt aus:

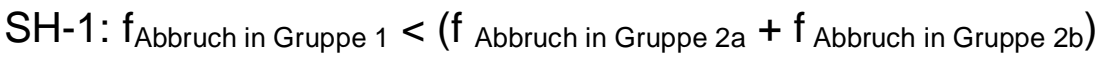


Die übrigen klinischen Hypothesen benennen Kovariationen bzw. Häufigkeitsverteilungen innerhalb der Gruppe 2a, die das Video gesehen hat.

Hypothese zur inneren Verarbeitung (KH-2)

KH-2:

Unter der Annahme, dass ein mittleres $M a ß$ an Furchtstärke nicht überschritten wird und Bewältigungsstrategien vermittelt werden, gilt:

Die innere Verarbeitung des Furchtappells steht in Zusammenhang mit dem Durchhaltevermögen der Rezipienten - je mehr aversive emotionale Schemata aktiviert werden, um so eher wird das Durchhaltevermögen positiv beeinflusst

Hinsichtlich der Klinischen Vorhersage bedeutet dies:

KV-2: .

Das Maß der inneren Verarbeitung des Furchtappells steht in einem negativen Zusammenhang mit dem Abbruch des stationären Drogenentzugs.

Erschöpfend wird diese Vorhersage auf die statistische Ebene durch folgende statistische Hypothese abgebildet:

$\mathrm{SH}-2 .: \mathrm{r}_{\mathrm{IVXAbbruch}}<0$

Hypothese zur zusätzlichen Induktion von Suchtdruck (KH-3)

KH-3:

Der Furchtappell erzeugt keinen zusätzlichen Suchtdruck.

Daraus lässt sich unter Berücksichtigung der Operationalisierung folgende klinische Vorhersage ableiten: 
KV-3:

Die Auswahl-Häufigkeiten der verschiedenen Stufen des erzeugten Suchtdrucks unterscheiden sich: Die Häufigkeit der Angabe, dass das Video keinen zusätzlichen Suchtdruck erzeuge, ist gröBer als die Häufigkeiten der übrigen Antwortalternativen.

SH-3: $f_{\text {ltem } 4=0}>f_{\text {ltem } 4=1} \cap f_{\text {ltem } 4=0}>f_{\text {ltem } 4=2}$

Hypothese zu geschlechtsspezifischen Unterschieden (KH-4)

KH-4:

Die innere Verarbeitung eines Furchtappells weist geschlechtsspezifische Unterschiede auf: Frauen werden durch den videogestützten Furchtappell eher gefühlsmäßig berührt als Männer.

Es ergibt sich daher die folgende klinische Vorhersage:

KV-4:

Es lässt sich ein negativer Zusammenhang feststellen zwischen der Ausprägung des Geschlechts und dem Maß der inneren Verarbeitung eines Furchtappells im stationären Drogenentzug.

Die statistische Hypothese lautet entsprechend:

SH-4: $\mathrm{r}_{\mathrm{IV} \times \mathrm{Geschlecht}}<0$

Alle Hypothesen, die Häufigkeitsunterschiede betreffen, werden mit einem 2- bzw. 4-Felder-Chi2-Test statistisch geprüft. Für die Überprüfung der Zusammenhangshypothesen werden Korrelationstests durchgeführt.

Die Datenanalysen erfolgen mittels des Computerprogramms SPSS $12.0^{\circledR}$ für Windows. 
Folgende Grundsätze finden bei der Überprüfung und Darstellung eventuell bestehender Gruppenunterschiede im Rahmen der nun folgenden Stichprobenbeschreibung (siehe Tabelle 2, S. 70 f.) Anwendung:

Häufigkeitsunterschiede von Merkmalen werden mittels Chi²-Tests ermittelt. Für ordinalskalierte Daten werden U-Tests nach Mann und Whitney angewendet. Der $\mathrm{t}$-Test ist für einen Mittelwertsvergleich bei intervallskalierten Daten sehr effizient (Bortz, 1999) und sollte daher verwendet werden, sofern die Varianzhomogenität der zu vergleichenden Gruppe gegeben ist. Ist die Annahme der Varianzhomogenität verletzt, dann sollte im Einzelfall entschieden werden, ob ein t-Test für heterogene Varianzen sinnvoll ist: Die alternative Verwendung des nicht parametrischen U-Tests nach Mann und Whitney ist dann angebracht, wenn die kleinere Gruppe die höhere Varianz aufweist, da dieses zu einer progressiven Testentscheidung führen kann, d.h. die Annahme der Alternativhypothese würde in diesem Fall erleichtert (Bortz, 1999). Dieses sollte allerdings vermieden werden. Die geschichtete Randomisierung führte zu der folgenden Gruppenbildung, die in Tabelle 2 (S. 70 f.) im Sinne einer Stichprobenbeschreibung dargestellt ist.

Alle Signifikanzen wurden dabei zweiseitig geprüft, „**“ bedeutet, ein hochsignifikanter Unterschied liegt vor.

\section{Tabelle 2: Stichprobenbeschreibung}

\begin{tabular}{|l|l|l|l|l|}
\hline $\begin{array}{l}\text { Merkmal / } \\
\text { Variable }\end{array}$ & EG & KG & Test & Statistiken \\
\hline \multicolumn{5}{|l|}{} \\
Soziodemographische Angaben \\
\hline Geschlecht & $\begin{array}{l}\mathrm{fm}=40 \\
\mathrm{fw}=20\end{array}$ & $\begin{array}{l}\mathrm{fm}=40 \\
\mathrm{fw}=20\end{array}$ & $\begin{array}{l}\text { 4-Felder } \\
\text { Chi'-Test }\end{array}$ & $\begin{array}{l}\mathrm{Chi}^{2}=0,000 \\
\mathrm{df}=1 \\
\mathrm{p}=1\end{array}$ \\
\hline Alter & $\mathrm{M}=29,22$ & $\begin{array}{l}\mathrm{M}=29,97 \\
\mathrm{~S}=7,114\end{array}$ & t-Test & $\begin{array}{l}\mathrm{t}=0,592 \\
\mathrm{df}=118 \\
\mathrm{p}=0,555\end{array}$ \\
\hline
\end{tabular}




\begin{tabular}{|c|c|c|c|c|}
\hline $\begin{array}{l}\text { Merkmal / } \\
\text { Variable }\end{array}$ & EG & KG & Test & Statistiken \\
\hline Familienstand $^{4}$ & $\begin{array}{l}f 0=45 \\
f 1=8 \\
f 2=6 \\
f 3=1\end{array}$ & $\begin{array}{l}f 0=41 \\
f 1=4 \\
f 2=12 \\
f 3=3\end{array}$ & U-Test & $\begin{array}{l}\text { Mittlerer Rang EG } \\
=57,66 \\
\text { Mittlerer Rang KG } \\
=63,34 \\
U=1629,5 \\
p=0,259\end{array}$ \\
\hline Partnerschaft & $\begin{array}{l}\text { fnein=29 } \\
\text { fja }=31\end{array}$ & $\begin{array}{l}\text { fnein }=26 \\
\mathrm{fja}=34\end{array}$ & $\begin{array}{l}\text { 4-Felder } \\
\text { Chi'-Test }^{2}\end{array}$ & $\begin{array}{l}\mathrm{Chi}^{2}=0,302 \\
\mathrm{df}=1 \\
\mathrm{p}=0,583\end{array}$ \\
\hline Kinder & $\begin{array}{l}\text { fnein }=35 \\
\mathrm{fja}=25\end{array}$ & $\begin{array}{l}\text { fnein }=40 \\
\mathrm{fja}=20\end{array}$ & $\begin{array}{l}\text { 4-Felder } \\
\text { Chi²Test }\end{array}$ & $\begin{array}{l}\mathrm{Chi}^{2}=0,889 \\
\mathrm{df}=1 \\
\mathrm{p}=0,346\end{array}$ \\
\hline Schulabschluss $^{5}$ & $\begin{array}{l}f 0=12 \\
f 1=29 \\
f 2=11 \\
f 3=8\end{array}$ & $\begin{array}{l}f 0=12 \\
f 1=30 \\
f 2=14 \\
f 3=4\end{array}$ & U-Test & $\begin{array}{l}\text { Mittlerer Rang EG } \\
=61,47 \\
\text { Mittlerer Rang KG } \\
=59,53 \\
\begin{array}{l}\mathrm{U}=1742 \\
\mathrm{p}=0,743\end{array}\end{array}$ \\
\hline $\begin{array}{l}\text { abgeschlossene } \\
\text { Ausbildung }\end{array}$ & $\begin{array}{l}\text { fnein }=38 \\
\mathrm{fja}=22\end{array}$ & $\begin{array}{l}\text { fnein }=36 \\
\mathrm{fja}=24\end{array}$ & $\begin{array}{l}\text { 4-Felder } \\
\text { Chi²Test }^{2}\end{array}$ & $\begin{array}{l}\text { Chi }^{2}=0,141 \\
d f=1 \\
p=0,707\end{array}$ \\
\hline Arbeit & $\begin{array}{l}\text { fnein }=54 \\
\text { fja }=6\end{array}$ & $\begin{array}{l}\text { fnein }=58 \\
\mathrm{f} j a=2\end{array}$ & $\begin{array}{l}\text { 4-Felder } \\
\text { Chi²Test }\end{array}$ & $\begin{array}{l}\text { Chi }^{2}=2,143 \\
d f=1 \\
p=0,143\end{array}$ \\
\hline $\begin{array}{l}\text { Wartezeitraum in } \\
\text { Tagen }\end{array}$ & $\begin{array}{l}M=66,45 \\
S= \\
46,891\end{array}$ & $\begin{array}{l}M=43,1 \\
S=39,821\end{array}$ & t-Test & $\begin{array}{l}t=-2,94 \\
d f=18 \\
p=0,004^{\star *}\end{array}$ \\
\hline \multicolumn{5}{|c|}{ Art der Abhängigkeit: } \\
\hline Polytoxikomanie & $\begin{array}{l}f=39 \text { (in } \\
\% ?)\end{array}$ & $f=33$ (in \%?) & $\begin{array}{l}\text { 2-Felder } \\
\text { Chi²Test }\end{array}$ & $\begin{array}{l}\text { Chi }^{2}=0,5 \\
d f=1 \\
p=0,48\end{array}$ \\
\hline $\begin{array}{l}\text { Opiat- } \\
\text { abhängigkeit }\end{array}$ & $\begin{array}{l}f=60 \\
(100 \%)\end{array}$ & $f=60(100 \%)$ & $\begin{array}{l}\text { 2-Felder } \\
\text { Chi²Test }\end{array}$ & $\begin{array}{l}\text { Chi }{ }^{2}=0 \\
d f=1 \\
p=1\end{array}$ \\
\hline $\begin{array}{l}\text { Benzodiazepin- } \\
\text { abhängigkeit }\end{array}$ & $f=34$ & $f=31$ & $\begin{array}{l}\text { 2-Felder } \\
\text { Chi²Test }\end{array}$ & $\begin{array}{l}\text { Chi }^{2}=0,138 \\
\text { df }=1 \\
p=0,710\end{array}$ \\
\hline $\begin{array}{l}\text { Kokain- } \\
\text { abhängigkeit }\end{array}$ & $f=12$ & $f=12$ & $\begin{array}{l}\text { 2-Felder- } \\
\text { Chi'-Test }\end{array}$ & $\begin{array}{l}\mathrm{Chi}^{2}=0,000 \\
\mathrm{df}=1 \\
p=1,000\end{array}$ \\
\hline $\begin{array}{l}\text { Alkohol- } \\
\text { abhängigkeit }\end{array}$ & $f=19$ & $f=18$ & $\begin{array}{l}\text { 2-Felder- } \\
\text { Chi'2-Test }\end{array}$ & $\begin{array}{l}\text { Chi }^{2}=0,027 \\
\text { df }=1 \\
p=0,869\end{array}$ \\
\hline
\end{tabular}

${ }^{4}$ Familienstand: $0=$ ledig; 1 = verheiratet; $2=$ geschieden; $3=$ verwitwet; $4=$ getrennt lebend

5: $0=$ kein Abschluss, $1=$ Hauptschulaabschluss, $2=$ Mittlere Reif, $3=$ Abitur 


\begin{tabular}{|c|c|c|c|c|}
\hline $\begin{array}{l}\text { Merkmal / } \\
\text { Variable }\end{array}$ & EG & KG & Test & Statistiken \\
\hline $\begin{array}{l}\text { THC- } \\
\text { Abhängigkeit }\end{array}$ & $f=25$ & $\begin{array}{l}\text { Schulab- } \\
\text { schluss } f=26\end{array}$ & $\begin{array}{l}\text { 2-Felder- } \\
\text { Chi²-Test }\end{array}$ & $\begin{array}{l}\text { Chi }^{2}=0,020 \\
d f=1 \\
p=0,889\end{array}$ \\
\hline $\begin{array}{l}\text { Amphetamin- } \\
\text { abhängigkeit }\end{array}$ & $f=0$ & $f=0$ & $-/-$ & $-/-$ \\
\hline \multicolumn{5}{|l|}{ Art des Entzuges: } \\
\hline Opiatentzug & $f=45$ & $f=49$ & $\begin{array}{l}\text { 2-Felder- } \\
\text { Chi'-Test }\end{array}$ & $\begin{array}{l}\mathrm{Chi}^{2}=0,170 \\
\mathrm{df}=1 \\
\mathrm{p}=0,680\end{array}$ \\
\hline $\begin{array}{l}\text { Benzodiazepin- } \\
\text { entzug }\end{array}$ & $f=37$ & $f=34$ & $\begin{array}{l}\text { 2-Felder- } \\
\text { Chi'-Test }\end{array}$ & $\begin{array}{l}\text { Chi }^{2}=0,127 \\
d f=1 \\
p=0,722\end{array}$ \\
\hline Kokainentzug & $f=18$ & $f=14$ & $\begin{array}{l}\text { 2-Felder- } \\
\text { Chi2-Test }\end{array}$ & $\begin{array}{l}\text { Chi }{ }^{2}=0,500 \\
d f=1 \\
p=0,480\end{array}$ \\
\hline Cannabisentzug & $f=32$ & $f=29$ & $\begin{array}{l}\text { 2-Felder- } \\
\text { Chi2-Test }\end{array}$ & $\begin{array}{l}\text { Chi }^{2}=0,148 \\
d f=1 \\
p=0,701\end{array}$ \\
\hline Alkoholentzug & $f=16$ & $f=158$ & $\begin{array}{l}\text { 2-Felder- } \\
\text { Chi'-Test }\end{array}$ & $\begin{array}{l}\text { Chi }^{2}=0,118 \\
d f=1 \\
p=0,732\end{array}$ \\
\hline Nulltag erreicht & $f=40$ & $f=43$ & $\begin{array}{l}\text { 2-Felder- } \\
\text { Chi2-Test }\end{array}$ & $\begin{array}{l}\text { Chi }^{2}=0,108 \\
d f=1 \\
p=0,742\end{array}$ \\
\hline $\begin{array}{l}\text { Frühabbrüche in } \\
\text { den ersten } 7 \\
\text { Tagen }\end{array}$ & $f=5$ & $f=4$ & $\begin{array}{l}\text { 2-Felder- } \\
\text { Chi2-Test }\end{array}$ & $\begin{array}{l}\text { Chi }^{2}=0,111 \\
d f=1 \\
p=0,739\end{array}$ \\
\hline $\begin{array}{l}\text { Frühabbrüche in } \\
\text { den ersten } 3 \\
\text { Tagen }\end{array}$ & $f=3$ & $f=1$ & $-/-$ & $p-0,1,0$ \\
\hline $\begin{array}{l}\text { Liegedauer in } \\
\text { Tagen }\end{array}$ & $\begin{array}{l}M=26,07 \\
S=13,508) \\
M i n=1 \\
M a x=66\end{array}$ & $\begin{array}{l}M=23,45 \\
(S=12,294) \\
M i n=1 \\
M a x=56 \\
\end{array}$ & t-Test & $\begin{array}{l}t=1,11 \\
d f=118 \\
p=0,269\end{array}$ \\
\hline Ziele $^{6}$ & $\begin{array}{l}\mathrm{f} 1=26 \\
\mathrm{f} 2=7 \\
\mathrm{f} 3=7 \\
\mathrm{f} 4=5 \\
\mathrm{f} 5=15\end{array}$ & $\begin{array}{l}f 1=20 \\
f 2=0 \\
f 3=10 \\
f 4=8 \\
f 5=22\end{array}$ & U-Test & $\begin{array}{l}\text { Mittlerer Rang KG } \\
=65,83 \\
\text { Mittlerer Rang EG } \\
=55,17 \\
\begin{array}{l}\mathrm{U}=1480,0 \\
\mathrm{p}=0,078\end{array}\end{array}$ \\
\hline $\begin{array}{l}\text { Anzahl vorheriger } \\
\text { LZT }\end{array}$ & $\begin{array}{l}M=0,92 \\
S=1,078\end{array}$ & $\begin{array}{l}M=0,93 \\
S= \\
0,1539\end{array}$ & $\mathrm{t}$-Test & $\begin{array}{l}t=0,069 \\
d f=118 \\
p=0,945\end{array}$ \\
\hline
\end{tabular}

${ }^{6}$ Ziele: $1=$ Langzeittherapie; $2=$ ambulante Therapie; $3=$ Erfüllen einer Therapieauflage gemäß $§ 35$ BtmG; 4=einer Arbeit nachgehen; 5=sonstige 


\begin{tabular}{|l|l|l|l|l|}
\hline $\begin{array}{l}\text { Merkmal } / \\
\text { Variable }\end{array}$ & EG & KG & Test & Statistiken \\
\hline $\begin{array}{l}\text { Anzahl er- } \\
\text { folgreicher LZT }\end{array}$ & $\begin{array}{l}\mathrm{M}=0,37 \\
\mathrm{~S}=0,663\end{array}$ & $\begin{array}{l}\mathrm{M}=0,42 \\
\mathrm{~S}=0,809\end{array}$ & $\mathrm{t}$-Test & $\begin{array}{l}\mathrm{t}=0,370 \\
\mathrm{df}=118 \\
\mathrm{p}=0,712\end{array}$ \\
\hline $\begin{array}{l}\text { Anzahl bisheriger } \\
\text { Entzüge }\end{array}$ & $\begin{array}{l}\mathrm{M}=5,65 \\
\mathrm{~S}=7,666\end{array}$ & $\begin{array}{l}\mathrm{M}=6,35 \\
\mathrm{~S}=8,178\end{array}$ & $\mathrm{t}$-Test & $\begin{array}{l}\mathrm{t}=0,484 \\
\mathrm{df}=118 \\
\mathrm{p}=0,629\end{array}$ \\
\hline $\begin{array}{l}\text { Anzahl er- } \\
\text { folgreicher Ent- } \\
\text { züge }\end{array}$ & $\begin{array}{l}\mathrm{M}=2,72 \\
\mathrm{~S}=4,088\end{array}$ & $\begin{array}{l}\mathrm{M}=3,75 \\
\mathrm{~S}=5,144\end{array}$ & t-Test & $\begin{array}{l}\mathrm{t}=1,218 \\
\mathrm{df}=118 \\
\mathrm{p}=0,226\end{array}$ \\
\hline $\begin{array}{l}\text { Substitution bei } \\
\text { Aufnahme }\end{array}$ & $\mathrm{f}=52$ & $\mathrm{f}=49$ & $\begin{array}{l}\text { 2-Felder- } \\
\text { Chi'-Test }\end{array}$ & $\begin{array}{l}\text { Chi }{ }^{2}=0,089 \\
\mathrm{df}=1 \\
\mathrm{p}=0,765\end{array}$ \\
\hline $\begin{array}{l}\text { Substitution bei } \\
\text { Entlassung }\end{array}$ & $\mathrm{f}=24$ & $\mathrm{f}=22$ & $\begin{array}{l}\text { 2-Felder- } \\
\text { Chi'-Test }\end{array}$ & $\begin{array}{l}\text { Chi' }=0,087 \\
\mathrm{df}=1 \\
\mathrm{p}=0,768\end{array}$ \\
\hline
\end{tabular}




\subsubsection{Darstellung der im Untersuchungszeitraum stationär aufgenommenen Patienten, die nicht in die Studie ein- geschlossen werden konnten.}

Im Untersuchungszeitraum wurden insgesamt 311 Fälle (301 Patienten $=207$ Männer und 94 Frauen) auf den Drogenentzugsstationen für illegal drogenabhängige Patienten behandelt. Davon wurden 120 Patienten in die Studie eingeschlossen (60 Patienten der Kontrollgruppe und 60 Patienten der Experimentalgruppe im Geschlechterverhältnis von je 40 Männern und 20 Frauen). Ausgeschlossen wurden 191 Fälle (181 Patienten; davon 127 Männer und 54 Frauen). Tabelle 3 (S. 74) gibt Aufschluss darüber, welche Kriterien dem Studienausschluss jeweils zugrunde lagen.

Tabelle 3: Ausschlussgründe im Überblick

\begin{tabular}{|l|c|c|c|c|}
\hline Zum Ausschluss führendes Merkmal & Fälle & $\begin{array}{l}\text { Patien- } \\
\text { ten }\end{array}$ & Männer & Frauen \\
\hline Akute Psychose & 6 & 6 & 5 & 1 \\
\hline Schwangerschaft & 5 & 5 & 0 & 5 \\
\hline Minderjährigkeit & 6 & 4 & 1 & 3 \\
\hline Einwilligungsunfähig & 1 & 1 & 1 & 0 \\
\hline Einwilligungsunwillig & 16 & 16 & 13 & 3 \\
\hline Unklare Behandlungsindikation & 8 & 8 & 7 & 1 \\
\hline Aufnahme durch AVD Wochenende/ nachts & 10 & 10 & 9 & 1 \\
\hline Aufnahme als interne Verlegung & 47 & 42 & 28 & 14 \\
\hline Erfüllten das Protokoll nicht & 42 & 42 & 31 & 11 \\
\hline Dosisreduktion bei Substitution & 13 & 12 & 8 & 4 \\
\hline Substitutionsumstellung auf Buprenorphin & 7 & 7 & 4 & 3 \\
\hline $\begin{array}{l}\text { Abgabe einer sauberen Urinkontrolle vor } \\
\text { Langzeittherapie }\end{array}$ & 24 & 23 & 16 & 7 \\
\hline Aufnahme zur Krisenintervention & 5 & 5 & 4 & 1 \\
\hline Summe & 191 & 181 & 127 & 54 \\
\hline
\end{tabular}




\section{Ergebnisse}

Es wird nun ausführlich dargestellt, zu welchen Ergebnissen die statistischen $\mathrm{Hy}$ pothesenüberprüfungen führen, um zu entscheiden, ob sich die klinischen Hypothesen bewähren. Vorab gilt es allerdings zu betrachten, ob eine experimentelle Variation des Furchtappells sowie die Konstruktion des Fragebogens zur Erfassung der inneren Verarbeitung ausreichend geglückt sind. Anschließend wird ebenfalls skizziert, wie hoch die teilnehmenden Patienten den subjektiven Nutzen des untersuchten Furchtappellvideos einschätzen.

\subsection{Experimentelle Variation des Furchtappells}

\section{Ist die Gruppenbildung geglückt?}

Es war geplant, bei Abbruchwunsch oder spätestens am Nulltag den Personen der Experimentalgruppe das Video der Aufnahmesituation zu zeigen, welches ihren ursprünglichen Allgemeinzustand dokumentierte. Allerdings waren trotz vorheriger Zustimmung nicht alle Teilnehmer dieser Gruppe ohne weiteres bereit, sich das Video dann auch tatsächlich anzusehen. Einige lehnten die Videokonfrontation kategorisch ab. In der folgenden Tabelle 4 (S. 76) ist zu sehen, dass sich etwa ein Drittel der Patienten trotz eingehender Bemühungen von ärztlicher Seite dagegen entschieden, das Video wie verabredet anzusehen. 
Tabelle 4: Gruppengrößen

\begin{tabular}{|c|c|c|c|c|c|}
\hline \multicolumn{4}{|c|}{$\begin{array}{l}\text { Experimentalgruppe mit Videodokumentation: } \\
N=60\end{array}$} & \multicolumn{2}{|c|}{$\begin{array}{l}\text { Kontrollgruppe ohne } \\
\text { Videodokumentation: } \\
\mathrm{N}=60\end{array}$} \\
\hline \multicolumn{2}{|c|}{$\begin{array}{l}\text { mit Videokonfrontation } \\
N=41\end{array}$} & \multicolumn{2}{|c|}{$\begin{array}{l}\text { ohne Videokonfrontation } \\
\mathrm{N}=19\end{array}$} & & \\
\hline $\begin{array}{l}0 \text {-Tag nicht } \\
\text { erreicht: } \\
\mathrm{N}=3\end{array}$ & $\begin{array}{l}\begin{array}{l}0-T a g \\
\text { erreicht } \\
\mathrm{N}=38\end{array} \\
\end{array}$ & \begin{tabular}{|l|}
$0-T a g$ \\
nicht \\
erreicht: \\
$\mathrm{N}=17$
\end{tabular} & $\begin{array}{l}\text { 0-Tag } \\
\text { erreicht: } \\
\mathrm{N}=2\end{array}$ & $\begin{array}{l}0-\text { Tag } \\
\text { nicht er- } \\
\text { reicht } \\
\mathrm{N}=17 \\
\end{array}$ & $\begin{array}{l}0-T a g \\
\text { erreicht } \\
N=43\end{array}$ \\
\hline
\end{tabular}

In der Gruppe mit Videodokumentation und -konfrontation sahen 3 Patienten (2 Männer und eine Frau) ihr Video bei Abbruchwunsch bereits vor dem Nulltag an. Dies führte jedoch nicht dazu, dass sie ihren Entschluss änderten, sondern sie brachen die Entgiftungsbehandlung dennoch ab.

Der Anteil der Experimentalgruppe, die die Videokonfrontation ablehnte, bestand aus 19 Patienten, davon 18 Abbrecher und ein erfolgreicher Entgifter. Dieser und noch ein anderer erreichten den Nulltag. Alle anderen 17 Probanden brachen die Behandlung vor dem Nulltag ab.

Ein Aspekt kristallisierte sich deutlich heraus: Keiner der Patienten, die die Entgiftung vor dem Nulltag abbrechen wollten, ließ sich von diesem Vorhaben abhalten. Sie waren kaum gesprächsbereit und wollten in den meisten Fällen das Video nicht anschauen. Stattdessen waren sie in der Regel bestrebt, das Krankenhaus so schnell wie möglich zu verlassen.

Doch auch, wenn die Videokonfrontation wie geplant statt fand, ergaben sich bei 2 Patienten gewisse Schwierigkeiten, diese Feedbacksitzung wie geplant durchführen: Sie brachen die Feedbacksitzung mitten drin ab und gaben an, dass eine Fortsetzung der Sitzung innen nichts bringen würde und dass sie keine Lust mehr hätten, das Video weiter anzuschauen. Beide waren ohnehin schwer zu motivieren gewesen, die Feedbacksitzung durchzuführen.

- Ein Teilnehmer deutschrussischer Abstammung, der die Sitzung nach 5 Minuten abbrach und den Raum verließ, gab im anschließenden Fragebo- 
gen an, dass inm das Video sehr peinlich sei. Eine Bearbeitung der Schamreaktion war im Rahmen eines therapeutischen Gesprächs mit der Ärztin jedoch nicht möglich. Er beendete die Entgiftungsbehandlung schließlich aber mit Erfolg.

- Der andere Rezipient gehörte zu den Dreien, die ihr Video bei Abbruchwunsch vor dem Nulltag anschauten. Er brach die Feedbacksitzung ab und beendete die Entgiftungsbehandlung noch am gleichen Tag gegen ärztlichen Rat.

\subsection{Der Fragebogen zur Erfassung der inneren Verarbei- tung}

Es wurde angenommen, dass mittels der formulierten Items das allgemeine Ausmaß aversiv erlebter gefühlsmäßiger Berührung erfasst wird. Diese Annahme wurde zunächst geprüft mittels einer Faktorenanalyse, da sie eine wesentliche Basis der weiteren Analysen darstellt. In diese Analyse wurde das zusätzliche Item zur Erfassung des Suchtdrucks einbezogen, welches einzeln auf einem unabhängigen Faktor laden sollte.

Die Faktorenanalyse wurde mittels SPSS ${ }^{\circledR} 12.0$ durchgeführt.

Wider Erwarten wurde in der faktoriellen Analyse neben den erwarteten Faktoren (a) Aversive gefüh/smäßige Berührung und (b) Suchtdruck ein weiterer relevanter Faktor aufgedeckt: Aufgrund der Zuordnung der Items ist davon auszugehen, dass dieser das Ausmaß bestehender (c) Reaktanz ${ }^{7}$ erfasst.

Die Zuordnung der Items zu Faktoren gestaltet sich im Detail wie folgt:

\footnotetext{
${ }^{7}$ Der Problemkreis der Reaktanz wurde von Brehm (1966) in seiner Theorie der Reaktanz ausgiebig untersucht. Mit psychologischer Reaktanz ist dabei eine komplexe Abwehrreaktion gemeint, die als Widerstand gegen eine äußere oder innere Einschränkung (z.B. Verbote, psychischen Druck) auftritt. Dabei bezeichnet man mit Reaktanz im eigentlichen Sinne nicht das ausgelöste Verhalten, sondern die zugrunde liegende Motivation oder Haltung.
} 


\section{Faktor 1: Aversive gefühlsmäßige Berührung}

- Item 1: Das Video berührt mich gefühlsmäßig. $(+)^{*}$

- Item 3: Es ist mir peinlich. (+)

- Item 5: Es stößt mich ab. (+)

- Item 7: Es deprimiert mich. (+)

\section{Faktor 2: Reaktanz}

- Item 1: Das Video berührt mich gefühlsmäßig. (-)

- Item 2: Es ist mir gleichgültig. (+)

- Item 6: Ich finde es hilfreich, die Entgiftung durchzuhalten. (-)

- Item 8: Es hat für mich keine Bedeutung, diese Aussage stimmt. (+)

\section{Faktor 3:}

Item 4: Es macht mir Suchtdruck (+)

$[(+)=$ positive Faktorladung; $(-)=$ negative Faktorladung $]$

Nähere Informationen zur durchgeführten Faktorenanalyse befinden sich im Anhang A dieser Arbeit (s. S. 112 f.).

Für die benannten Faktoren wurden Summenwerte der Items gebildet, die eine Faktorladung $>$ oder $=|.5|$ für den jeweiligen Faktor haben.

Aufgrund der negativen Faktorladungen der Items 1 und 6 auf dem Faktor 2 Reaktanz wurden bei diesen Items die Skalen invers angelegt. Das bedeutet, "nicht" wurde der Wert 2 zugewiesen, „etwas“ der Wert 1 und „sehr“ der Wert 0.

So wurden im Folgenden also aversive gefühlsmäßige Berührung und Reaktanz als zwei unabhängig Subskalen betrachtet, Durchschnittswerte der beteiligten Items gebildet und in den Analysen diese Subskalengesamtwerte entsprechend getrennt einbezogen. 
In der nachstehenden Tabelle finden sich die Gruppen-Mittelwerte und Streuungen der beiden Subskalen sowie des Items 4 zur Erfassung des induzierten Suchtdrucks.

Tabelle 5: Deskriptive Statistiken zur inneren Verarbeitung

\begin{tabular}{|l|c|c|c|c|c|}
\hline & N & Minimum & Maximum & M & S \\
\hline $\begin{array}{l}\text { Gefühlsmäßige Berüh- } \\
\text { rung } \\
\text { [Subskala 1 (1,3,5,7)] }\end{array}$ & 38 & 0 & 2 &, 9671 &, 58751 \\
$\begin{array}{l}\text { Reaktanz } \\
\text { [Subskala 2 (-1,2,-6,8)] }\end{array}$ & 39 & 0 & 2 &, 4744 &, 47224 \\
$\begin{array}{l}\text { Induzierter Suchtdruck } \\
\text { [Einzel-Item 4] } \\
\begin{array}{l}\text { Gültige Werte } \\
\text { (Listenweise) }\end{array}\end{array}$ & 39 & 0 & 2 &, 21 &, 570 \\
\hline
\end{tabular}

Insgesamt erfasst der konstruierte Fragebogen wie gewünscht die aversive gefühlsmäßige Berührung. Darüber hinausgehend bildet er zudem einen Aspekt ab, der bislang wenig Berücksichtigung fand: Das Ausmaß bestehender Reaktanz.

\subsection{Statistische Hypothesenprüfungen}

\section{Statistische Überprüfung zur KH-1}

Wird das Durchhaltevermögen im stationären Drogenentzug erhöht durch das Aufzeigen des Allgemeinzustands bei der Krankenhausaufnahme?

Die statistische Hypothese zum Unterschied bezüglich der Abbruchhäufigkeit kann mittels eines 2-Felder-Chi2-Tests geprüft werden. Die Anzahl der Abbrüche sowie das Ergebnis der statistischen Analyse finden sich in Tabelle 6 bzw. Abbildung 11 (S. 80). 
Tabelle 6: Anzahl der Abbrüche in Kontroll- und Experimentalgruppe

\begin{tabular}{|l|c|c|c|}
\hline & & $\begin{array}{c}\text { mit Video- } \\
\text { dokumentation }\end{array}$ & \multirow{2}{*}{ Gesamt } \\
\cline { 2 - 3 } & $0(\mathrm{KG})$ & 1 (ExpG) & \\
\hline $\begin{array}{l}\text { Anzahl } \\
\text { Abbrüche }\end{array}$ & 27 & 27 & 54 \\
\hline
\end{tabular}

$\mathrm{Chi}^{2}=0,000 ; \mathrm{df}=1 ;$ Signifikanz $=1,000$

*Nicht alle dieser Personen sahen das Video an

Deutlich wird hier, dass die Abbruchraten beider Gruppen jeweils $45 \%$ betragen und sich somit in keiner Weise unterscheiden.

Die experimentelle Gruppenbildung führte zu keinen Unterschieden hinsichtlich der Abbruchhäufigkeit.

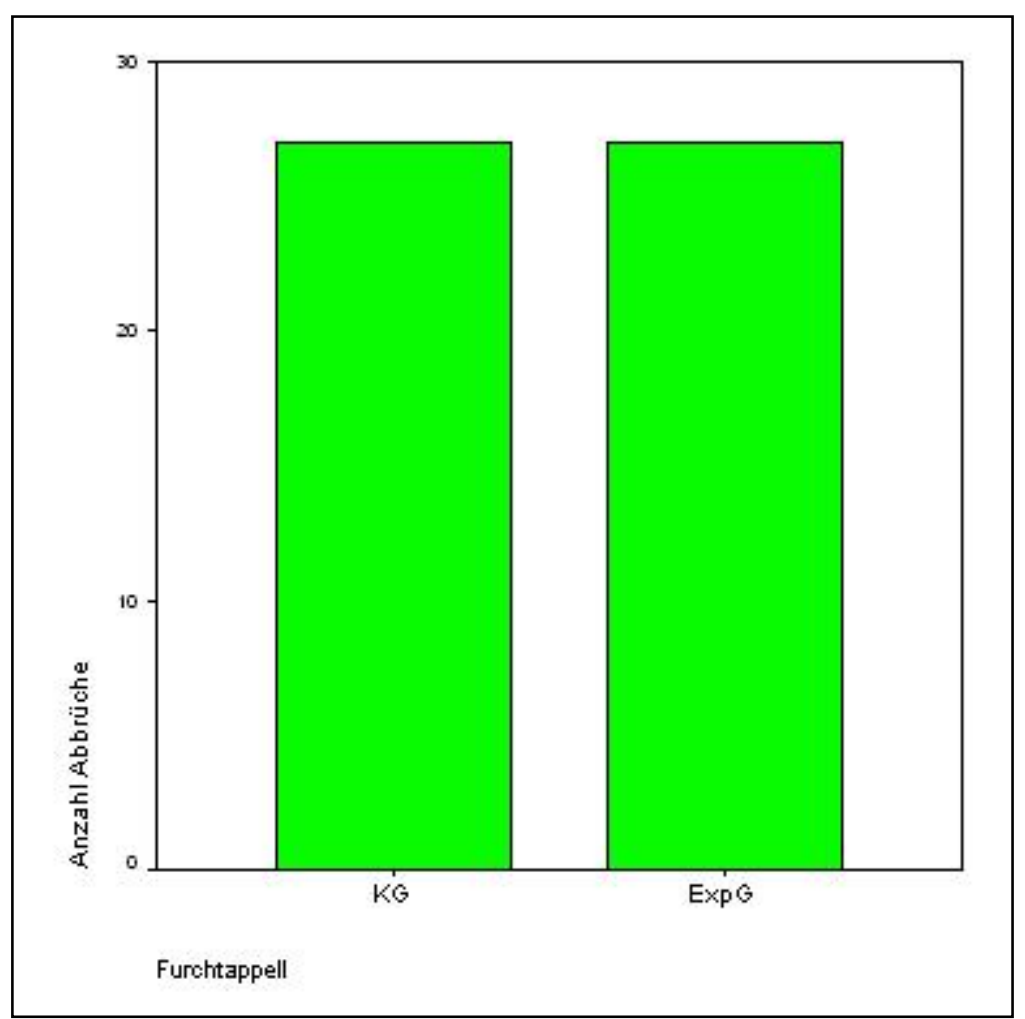

Abbildung 11: Anzahl der Abbrüche in Kontroll- (KG) und Experimentalgruppe (ExpG) 
$\rightarrow$ Die KH-1 kann daher als nicht bewährt angesehen werden.

Die Videodokumentation (und -konfrontation) als Furchtappell verbessert nicht generell das Durchhaltevermögen im stationären Drogenentzug.

\section{Ergänzendes zu KH-1:}

Es wurde bereits beschrieben, dass nicht alle Personen der Experimentalgruppe das Video tatsächlich auch ansahen: Das bedeutet, die Experimentalgruppe lässt sich in zwei Subgruppen aufteilen. Eine Gruppe erhielt nur die Videodokumentation, die anderen Teilnehmer dieser Gruppe erhielten sowohl eine Videodokumentation als auch eine -konfrontation. Die Experimentalgruppe unterschied sich demnach von der Kontrollgruppe mindestens dadurch, dass alle Rezipienten an der Dokumentation teilnahmen und wussten, sie würden dieses Video bei Abbruchwunsch oder am Nulltag ansehen.

Wie viele Patienten der Experimentalgruppe (mit Videodokumentation) sahen sich das Video nun tatsächlich an? Der folgenden Darstellung ist zu entnehmen, dass gut zwei Drittel der relevanten Personen einer Videokonfrontation auch tatsächlich zustimmten.

Tabelle 7: Darstellung der Subgruppen der Experimentalgruppe

\begin{tabular}{|l|c|c|}
\hline & Häufigkeit & Prozent \\
\hline Videokonfrontation abgelehnt & 19 & 31,7 \\
\cline { 1 - 1 } Videokonfrontation zugestimmt & 41 & 68,3 \\
\cline { 1 - 1 } Gesamt & 60 & 100,0 \\
\hline
\end{tabular}

Es ist vorstellbar, dass kein Unterschied zwischen Experimental- und Kontrollgruppe hinsichtlich der Abbruchhäufigkeit gefunden werden konnte aufgrund der fehlenden Videokonfrontation. Diese stellt einen essentiellen Bestandteil des intendierten Furchtappells dar. Um in dieser Hinsicht nähere Erkenntnisse zu gewinnen, wurden erneute Analysen für eine verkleinerte Stichprobe durchgeführt:

1. diejenigen, die tatsächlich das Video ansahen,

2. wurden den Patienten der KG hinsichtlich der Abbruch-Häufigkeit gegenübergestellt: 
Tabelle 8: Darstellung der Abbruchhäufigkeit in der Subgruppe mit Videokonfrontation und der Kontrollgruppe

\begin{tabular}{|l|c|c|c|}
\hline & & $\begin{array}{c}\text { mit Videokon- } \\
\text { frontation* }\end{array}$ & \multirow{2}{*}{ Gesamt-Anzahl } \\
\cline { 2 - 3 } & $0(\mathrm{KG})$ & $1($ ExpG) & \\
\hline Anzahl Abbrüche 1 & $27(45 \%)$ & $10(24,5 \%)$ & 37 \\
\hline kein Abbruch 0 & $33(55 \%)$ & $31(75,5 \%)$ & 64 \\
\hline Gesamt-Anzahl & $60(100 \%)$ & $41(100 \%)$ & 101 \\
\hline
\end{tabular}

$\mathrm{Chi}^{2}=4,457 ; \mathrm{df}=1 ;$ Signifikanz $=0,035$

${ }^{\star}$ Zu berücksichtigen: Diese Analyse gilt für eine verkleinerte Gesamt-Stichprobe!

In der Gruppe mit Videokonfrontation ist die Abbruchhäufigkeit signifikant geringer als in der Kontrollgruppe!

Für diejenigen, bei denen eine Videokonfrontation geplant war, wird ergänzend geprüft, inwieweit das Geschlecht das tatsächliche Ansehen des Videos vorhersagen kann. Die Analyse führt zu folgendem Ergebnis:

Tabelle 9: Abhängigkeit der Bereitschaft zur Videokonfrontation vom Geschlecht

\begin{tabular}{|l|c|c|c|}
\hline & \multicolumn{2}{|c|}{ Geschlecht } & \multirow{2}{*}{ Gesamt-Anzahl } \\
\cline { 2 - 3 } & $0=\mathrm{w}$ & $1=\mathrm{m}$ & 41 \\
\hline Videokonfrontation & 15 & 26 & 19 \\
\hline keine Videokonfrontation & 5 & 14 & 60 \\
\hline Gesamt-Anzahl & 20 & 40 & \\
\hline
\end{tabular}

$\mathrm{Chi}^{2}=0,616 ; \mathrm{df}=1 ;$ Signifikanz $=0,432$ 
Die Analysen zeigen keinen Häufigkeitsunterschied hinsichtlich der Videokonfrontation: Unter Berücksichtigung der nach dem Geschlechterverhältnis „Frauen I Männer" = 1 / 2 vorgenommenen Schichtung der Stichprobe sehen sich weder Frauen noch Männer eher das Video an (s. Abbildung 12, S. 83).

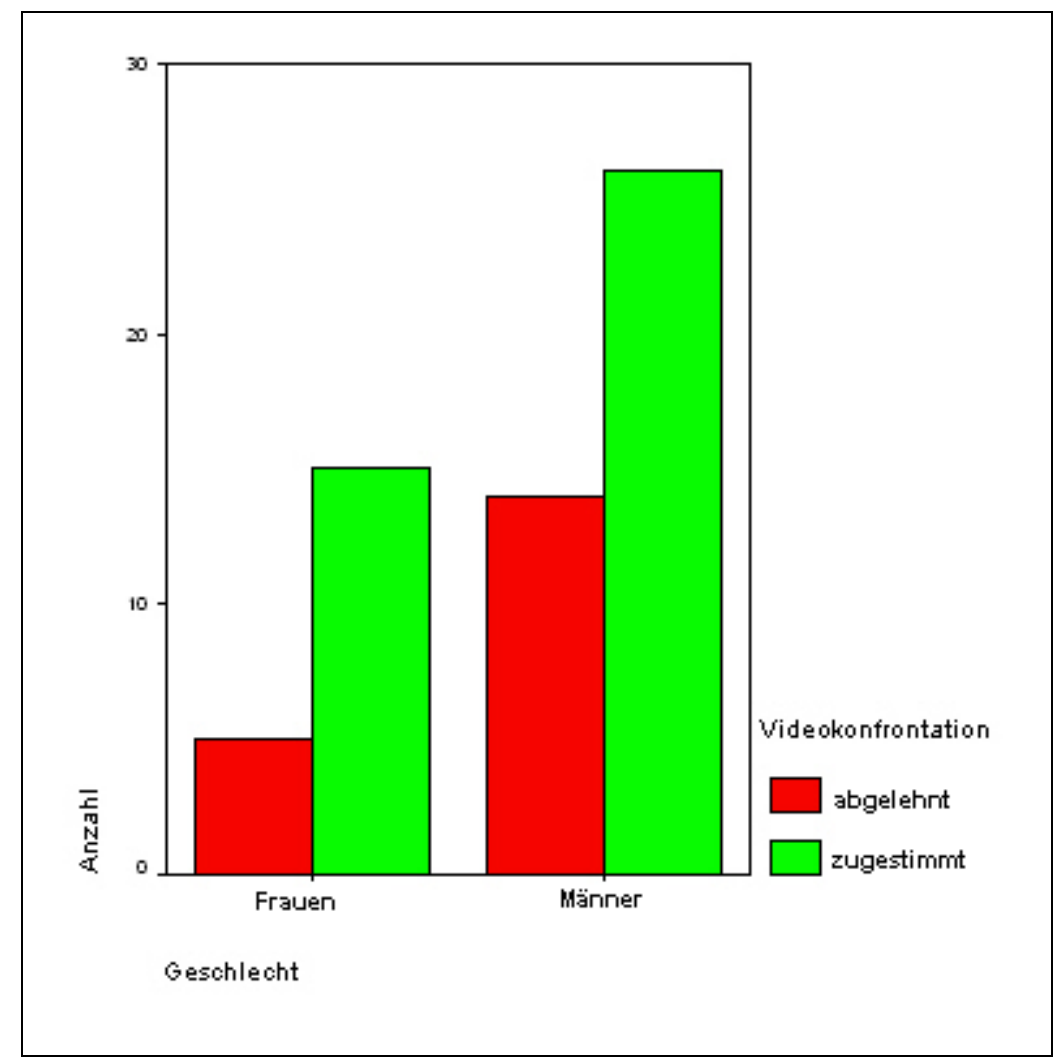

\section{Abbildung 12: Anzahl der Videokonfrontationen bei Männern und Frau- en}

\section{Statistische Überprüfung zur $\mathrm{KH}-2$}

Für die Subgruppe derer, die das Video sahen, wird nun der Fragebogen zur inneren Verarbeitung einbezogen.

Um die Zusammenhangs-Hypothese zu prüfen wurde zunächst nur Faktor 1 - Gefühlsmäßige Berührung berücksichtigt.

Die entsprechenden Skalensummenwerte des Fragebogens wurden korreliert mit der Variable Abbruch (Ja/Nein), um eventuell bestehende Zusammenhänge zwi- 
schen dem Maß der inneren Verarbeitung und dem Abbruch des Entzugs aufzudecken.

Wie den folgenden Darstellungen in Tabelle 10 (S. 84) zu entnehmen ist, wurde jedoch keine statistisch signifikante Korrelation aufgedeckt.

Tabelle 10: Zusammenhang zwischen Behandlungsabbruch und Ausmaß der inneren Berührung durch die Videokonfrontation

\begin{tabular}{|l|l|c|}
\hline & \multicolumn{1}{|c|}{$\begin{array}{c}\text { Durchschnittliche } \\
\text { gefühlsmäßige Berührung }\end{array}$} \\
\hline $\begin{array}{l}\text { Abbruch } \\
(1=\text { ja, } 0=\text { nein })\end{array}$ & $\begin{array}{l}\text { Korrelation nach } \\
\text { Pearson } \\
\text { Signifikanz } \\
(1 \text {-seitig }) \\
\mathrm{N}\end{array}$ &, 071 \\
\hline
\end{tabular}

Der angenommene Zusammenhang zwischen dem Ausmaß der gefühlsmäßigen Berührung durch den Furchtappell und der Anzahl der Abbrüche im stationären Drogenentzug konnte auf statistischer Ebene nicht abgebildet werden.

$\rightarrow \mathrm{KH}-2$ hat sich daher ebenfalls nicht bewährt. Eine stärkere aversive gefühlsmäßige Berührung geht nicht mit einem verbesserten Durchhaltevermögen einher.

\section{Ergänzendes zu KH-2:}

Überraschender Weise erfasste der Fragebogen neben dem Ausmaß GefühlsmäBiger Berührung auch das Ausmaß bestehender Reaktanz auf Seiten der Patienten. Es ist anzunehmen, das dieses in einem Zusammenhang steht mit dem Durchhaltevermögen der Patienten: Je stärker die Reaktanz, desto weniger kann das Durchhaltevermögen begünstigt werden durch den Furchtappell. Auf der statistischen Ebene sollte sich also ein positiver Zusammenhang zeigen zur Variable $\operatorname{Abbruch}(\mathrm{Ja}=1 / \mathrm{Nein}=0)$. 
Tabelle 11: Darstellung des Zusammenhangs zwischen Ausmaß der Reaktanz und dem Durchhaltevermögen in der stationären Entgiftung

\begin{tabular}{|l|l|c|}
\hline & & Durchschnittliche Reaktanz \\
\hline $\begin{array}{l}\text { Abbruch } \\
(1=\text { ja, } \\
0=\text { nein })\end{array}$ & Korrelation nach Pearson &, 056 \\
& Signifikanz (1-seitig) &, 3695 \\
& $\mathrm{~N}$ & 38 \\
\hline
\end{tabular}

Es lässt sich allerdings keine statistisch signifikante Korrelation zwischen diesen Variablen finden, d.h. auch diese klinische Hypothese hat sich nicht bestätigt. Zwischen der erfassten Reaktanz und der Abbruchhäufigkeit ist kein Zusammenhang erkennbar.

Statistische Überprüfung zur KH-3

Es gilt weiterhin die Annahme zu betrachten, dass durch das Ansehen des Videos kein zusätzlicher Suchtdruck erzeugt wird.

Die Häufigkeitsverteilung des Antwortverhaltens sieht hier wie folgt aus: 


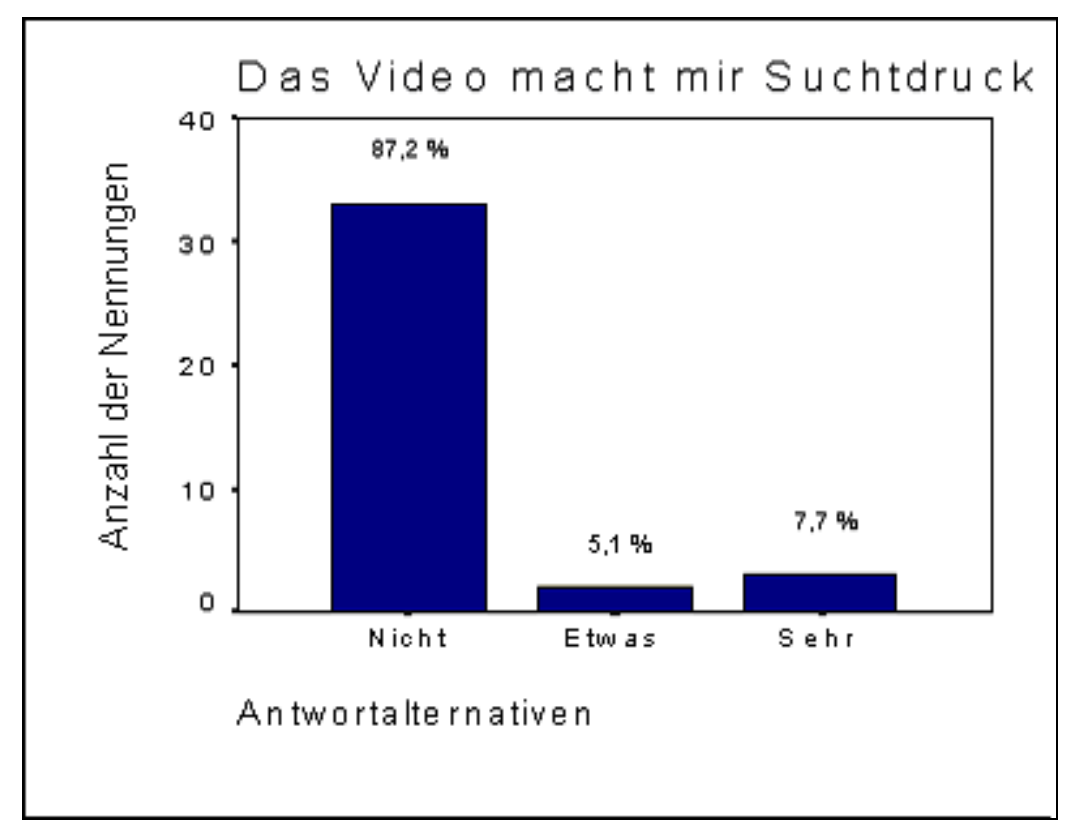

\section{Abbildung 13: Darstellung der Anzahl der Nennungen von Antwortal- ternativen zur Frage nach dem Suchtdruck infolge der Videokonfronta- tion}

Die Anzahl der Nennungen weichen signifikant von einer zufälligen Gleichverteilung ab (Chi² = 49,00; df = 2; asymptotische Signifikanz: 0,000).

92,3\% der Probanden geben im Fragebogen maximal an, dass das Video "etwas“ Suchtdruck erzeugt.

$\rightarrow \mathrm{KH}-3$ hat sich bewährt. Das Video erzeugt bei einem Großteil der Patienten keinen zusätzlichen Suchtdruck.

Es wäre zu erwarten, dass diejenigen, bei denen Suchtdruck erzeugt wird, den Furchtappell nicht in der erwünschten Richtung verarbeiten und daher ein Zusammenhang zum Durchhaltevermögen der Patienten besteht. Ist dieser Zusammenhang auf statistischer Ebene abzubilden? 
Tabelle 12: Darstellung des Zusammenhangs zwischen erzeugtem Suchtdruck und Durchhaltevermögen der Patienten

\begin{tabular}{|l|l|c|}
\hline & & Wert Item 4 \\
\hline $\begin{array}{l}\text { Abbruch } \\
(1=\text { ja, 0 = nein })\end{array}$ & Korrelation nach Pearson &, 191 \\
& Signifikanz (2-seitig) &, 258 \\
& $\mathrm{~N}$ & 37 \\
\hline
\end{tabular}

Diese Zusammenhangshypothese lässt sich auf statistischer Ebene nicht bestätigen, möglicherweise allerdings aufgrund der fehlenden Varianz in den Antworten.

\section{Statistische Überprüfung zur $\mathrm{KH}-4$}

Es wurde weiterhin eine Geschlechtsspezifität der inneren Verarbeitung, der individuellen inneren Reaktion, auf das Video angenommen: Es wurde davon ausgegangen, dass Frauen durch das Video stärker gefühlsmäßig berührt werden. Um diese Zusammenhangshypothese zu prüfen wurde auf statistischer Ebene eine Korrelation berechnet zwischen dem Skalensummenwert gefühlsmäßiger Berührung und dem Geschlecht. Es wurde eine signifikante negative Korrelation erwartet und auch gefunden!

Tabelle 13: Darstellung des Zusammenhangs zwischen Geschlecht und innerer Verarbeitung des Videos

\begin{tabular}{|l|l|c|}
\hline & & Gefühlsmäßige Berührung \\
\hline $\begin{array}{l}\text { Geschlecht } \\
(\mathrm{m}=1 / \mathrm{w}=0)\end{array}$ & Korrelation nach Pearson &,$- 333\left(^{*}\right)$ \\
\hline & $\begin{array}{l}\text { Signifikanz } \\
(1-\text { seitig })\end{array}$ &, 044 \\
\hline & $\mathrm{N}$ & 37 \\
\hline
\end{tabular}

* Die Korrelation ist auf dem Niveau von 0,05 (1-seitig) signifikant. 
$\rightarrow \mathrm{KH}-4$ hat sich demnach bewährt: Frauen werden durch den Furchtappell stärker aversiv-gefühlsmäßig berührt als Männer.

Southwick-Bensley und Wu (1991) zeigten bereits, dass Männer eher dazu tendieren, mit Reaktanz auf Vorsorgeempfehlungen zu reagieren. Dieser Zusammenhang zwischen Geschlecht und Reaktanz zeigt sich auch in der klinischen Erfahrung im Rahmen der Entgiftungsbehandlung. Aus statistischer Ebene sollte sich daher eine positive Korrelation zwischen den zugehörigen Variablen zeigen.

Diese lässt sich auch tatsächlich - zumindest tendenziell - finden.

Tabelle 14: Darstellung des Zusammenhangs zwischen Geschlecht und Reaktanz

\begin{tabular}{|l|l|c|}
\hline & & Reaktanz \\
\hline $\begin{array}{l}\text { Geschlecht } \\
(\mathrm{m}=1 / \mathrm{w}=0)\end{array}$ & Korrelation nach Pearson &, $316^{\star}$ \\
& $\mathrm{p}(1$-seitig) &, 054 \\
& $\mathrm{~N}$ & 38 \\
\hline
\end{tabular}

* Die Korrelation ist auf dem Niveau von 0,05 (1-seitig) signifikant.

$\rightarrow$ Männer reagieren tatsächlich stärker mit einer reaktanten Haltung, als Frauen es tun.

Ergänzend zu diesen Annahmen wurde des Weiteren betrachtet, ob sich ein geschlechtsspezifischer Unterschied hinsichtlich des erzeugten Suchtdrucks abbilden lässt. Es wäre davon auszugehen, dass bei Männern eher Suchtdruck erzeugt wird als bei Frauen, da sie den Furchtappell möglicherweise bei ausgeprägter Reaktanz in einer ungünstigen Weise verarbeiten. 
Auf statistischer Ebene ließe sich diese Zusammenhangs-Hypothese wiederum prüfen durch einen Korrelationstest. Erwartet wird eine statistisch bedeutsame positive Korrelation, die sich in dieser Form jedoch nicht finden lässt.

Tabelle 15: Darstellung des Zusammenhangs zwischen Geschlecht und Suchtdruck

\begin{tabular}{|l|l|c|}
\hline & & Item 4: ...erzeugt Suchtdruck \\
\hline $\begin{array}{l}\text { Geschlecht } \\
(m=1 / w=0)\end{array}$ & Korrelation nach Pearson &,$- 308^{*}$ \\
& $p(1$-seitig $)$ &, 063 \\
& $N$ & 37 \\
\hline
\end{tabular}

* Die Korrelation ist auf dem Niveau von 0,05 (1-seitig) signifikant.

Wie diesen Darstellungen zu entnehmen ist, weisen die Daten eher einen Trend in die entgegen gesetzte Richtung auf: Frauen entwickeln durch das Video induziert tendenziell einen stärkeren Suchtdruck.

Da nicht auszuschließen ist, dass der Fragebogen die geschlechtsspezifischen Unterschiede in der Reaktion auf das Video nur unzureichend erfasst, wurden für die Gruppe derer, die das Video sahen, sowie für die Gruppe ohne Videodokumentation jeweils Zusammenhänge zwischen den Variablen Geschlecht und $A b$ bruch geprüft. Erwartet wurden ein signifikanter Zusammenhang für die Gruppe mit Video (aufgrund geschlechtsspezifischer Unterschiede in der Verarbeitung des Furchtappells) und kein signifikanter Zusammenhang in der anderen Gruppe. 
Tabelle 16: Darstellung der Zusammenhänge zwischen Geschlecht und Abbruch für die Subgruppe mit Videokonfrontation

\begin{tabular}{|l|l|c|c|c|}
\hline \multicolumn{2}{|c|}{} & \multicolumn{2}{c|}{ Geschlecht } & \multirow{2}{*}{ Gesamt-Anzahl } \\
\cline { 3 - 4 } \multicolumn{2}{|c|}{ Abbruch } & Nein = 0 & $\mathbf{m}=\mathbf{1}$ & \\
\hline & Ja $=1$ & $9(60 \%)$ & $22(85 \%)$ & 31 \\
\hline Gesamt-Anzahl & $6(40 \%)$ & $4(15 \%)$ & 10 \\
\hline
\end{tabular}

$\mathrm{Chi}^{2}=3,125(\mathrm{~b}) ; \mathrm{df}=1$; Signifikanz = 0,07.

Für die Kontroll-Gruppe ohne Videodokumentation

(Hier wurden alle 60 Probanden einbezogen, da ein signifikanter Zusammenhang dadurch eher aufzudecken wäre):

Tabelle 17: Darstellung der Zusammenhänge zwischen Geschlecht und Abbruch für die Subgruppe ohne Videodokumentation (Kontrollgruppe)

\begin{tabular}{|c|c|c|c|c|}
\hline & \multicolumn{2}{|c|}{ Geschlecht } & \multirow{2}{*}{ Gesamt-Anzahl } \\
\hline & & $w=0$ & $m=1$ & \\
\hline \multirow[t]{2}{*}{ Abbruch } & Nein $=0$ & $11(55 \%)$ & $22(55 \%)$ & 33 \\
\hline & $\mathrm{Ja}=1$ & $9(45 \%)$ & $18(45 \%)$ & 27 \\
\hline \multicolumn{2}{|c|}{ Gesamt-Anzahl } & 20 & 40 & 60 \\
\hline
\end{tabular}

$\mathrm{Chi}^{2}=0,000(\mathrm{~b}) ; \mathrm{df}=1 ;$ Signifikanz $=1,000$

Die Darstellungen zeigen, dass sich tatsächlich in der Gruppe mit Video tendenziell ein Zusammenhang zwischen Geschlecht und Abbruch zeigt, der in der anderen Gruppe ohne Video so nicht aufzufinden ist. 

Furchtappells

Neben dem objektiven Maß Abbruch (Ja/Nein) als Operationalisierung des Durchhaltevermögens der Patienten in der stationären Drogenentgiftung wurde die Zielgruppe auch nach ihrer persönlichen Einschätzung des Nutzens des untersuchten Furchtappells befragt. Sie sollten dabei angeben, ob dieses Video bei allen Patienten im Drogenentzug standardmäßig eingesetzt werden sollte. Wie hilfreich schätzten die Teilnehmer der Studie das Video nun ein?

Tabelle 18: Darstellung der Antworten auf die Frage nach einem standardmäßigen Einsatz des Videobasierten Furchtappells im stationären

\section{Drogenentzug}

„Das Video sollte bei allen Patienten eingesetzt werden...“

\begin{tabular}{|l|l|l|}
\hline & Häufigkeit & Prozent \\
\hline Nein / & 5 & 13,2 \\
$\mathrm{Ja} / 1$ & 33 & 86,8 \\
Gesamt & 38 & 100,0 \\
\hline
\end{tabular}

Der deutliche Großteil der Probanden (86,8\%) hält es nach diesen Antworten zu urteilen für sinnvoll, dieses Video bei allen Patienten einzusetzen. Frauen würden eher das Video als Standard einsetzen als Männer (s. Tabelle 19 S. 91 ):

Tabelle 19: Einschätzung des Nutzens des Furchtappells bei Männern und Frauen. („Das Video sollte bei allen Patienten eingesetzt werden...“)

\begin{tabular}{|l|l|l|l|}
\hline & \multicolumn{2}{|l|}{ Geschlecht } & \multirow{2}{*}{ Gesamt } \\
\hline & $0=\mathrm{w}$ & $1=\mathrm{m}$ & \\
\hline Nein / & 0 & 5 & 5 \\
$\mathrm{Ja} / 1$ & 14 & 19 & 33 \\
Gesamt & 14 & 24 & 38 \\
\hline
\end{tabular}

$\mathrm{Chi}^{2}=3.359 ; \mathrm{df}=1 ;$ Signifikanz $=0,067$ 
Die praktischen Probleme der vorliegenden Studie werden nun eingehend beschrieben, sowohl im Hinblick auf die formulierten Hypothesen dargestellt wie auch auf die denkbare Umsetzung der Ergebnisse in die Praxis. Es folgt eine Diskussion der Befunde mit Rückgriff auf die empirischen und theoretischen Grundlagen.

\subsection{Repräsentativität der Stichprobe}

In der vorliegenden Arbeit wurde der Einfluss eines videobasierten Furchtappells auf das Durchhaltevermögen im stationären Drogenentzug untersucht. Oft sind Untersuchungen in der Furchtappellforschung unter Laborbedingungen (v. a. mit studentischen Probanden) durchgeführt worden. Um Einzeleffekte zu untersuchen kann dies durchaus sinnvoll sein. Die vorliegende Studie versuchte jedoch, den praktischen Nutzen der videogestützten Konfrontationssitzung bei suchtkranken Patienten direkt unter den realen Bedingungen der stationären Entgiftungsbehandlung zu untersuchen. Die gewählte Form der Umsetzung erscheint in diesem speziellen Fall günstiger, um praxisrelevante Ergebnisse zu erzielen.

Betrachtet man die soziodemographischen Daten, Arten der Suchterkrankungen sowie Arten und Häufigkeiten der Substitutionen, so wird deutlich, dass die Stichprobenauswahl eine Gesamtgruppe von Probanden hervorgebracht hat, die repräsentativ hinsichtlich der alläglichen Klientel der Entgiftungsstationen im ehemaligen NLKH Göttingen ist. Es handelt sich hierbei um schwerkranke Patienten, die neben ihrer Suchterkrankung eine Vielzahl körperlicher, sozialer oder interpersoneller und psychischer Beeinträchtigungen aufweist.

Es wurde eine Stichprobe von $N=60$ Patienten erhoben. Dabei wurde nach einem Randomisierungsplan eine geschichtete Randomisierung (2/3 Männer, 1/3 Frauen) gezogen. Das gewählte Geschlechterverhältnis entspricht erfahrungsgemäß dem Verhältnis von Männern und Frauen auf Drogen - Entgiftungsstationen (sie- 
he auch Tabelle 1, S. 4). Einen signifikanten Unterschied zwischen der Experimentalgruppe und der Kontrollgruppe gibt es nur hinsichtlich der Wartezeit. Dieser ist zwar im statistischen Sinne signifikant, praktisch betrachtet aber vermutlich wenig bedeutsam.

Alle Patienten hatten eine Opioidabhängigkeit. Die Diagnose einer Polytoxikomanie wurde bei 39 Patienten $(=57,5 \%)$ der Experimentalgruppe und 33 Patienten der Kontrollgruppe $(=52,5 \%)$ gestellt.

Der Altersdurchschnitt liegt in der Experimentalgruppe bei 29,2 und in der Kontrollgruppe bei 30,0 Jahren. Er liegt damit etwas höher als auf den Entgiftungsstationen, die in Tabelle 1 (S. 4) dargestellt sind. Dies deckt sich aber mit den Ergebnissen von Backmund et al. (2006), die die Klientel auf einer Drogenentzugsstation im Zeitraum von 1991-1996 sowie im Zeitraum von 2001-2005 verglichen und eine Zunahme des Altersdurchschnitts der Patienten von 28 auf 32 Jahre feststellten. Die Zunahme des Altersdurchschnitts könnte mit einer Zunahme methadonsubstituierter Patienten im Verlauf der Etablierung der Substitutionsprogramme zusammenhängen. In der aktuellen Untersuchung hatten 52 Probanden der Experimental- und 49 Probanden der Kontrollgruppe eine Substitutionstherapie bei Aufnahme in die Klinik. Ein häufiges Problem im Rahmen der Substitutionstherapien stellt der Nebenkonsum von Heroin (Hartel et al. 1995), Kokain (Dobler- Mikola et al. 2000; Leri et al. 2003), Benzodiazepinen (Backmund et al. 2005), Alkohol (Backmund et al. 2003) und auch Cannabis (Epstein und Preston 2003) dar. In Folge dessen werden auf den Entgiftungsstationen neben Opiatentgiftungen zunehmend auch Entgiftungen vom Nebenkonsum bei Methadon-substituierten Patienten durchgeführt. Isoliert heroinabhängige Patienten, die eine reine Opiatentgiftung durchführen wollen, sind selten geworden. 


\subsection{Diskussion zum Einfluss des videobasierten Furchtap- pells auf die Abbruchquote im stationären Drogenent- zug}

Behandlungsabbrüche stellen nach wie vor ein erhebliches Problem im Rahmen des stationären Drogenentzugs dar: Durch die Einführung des sogenannten „warmen" methadongestützten Opiatentzugs konnte lediglich die Rate an Frühabbrüchen in den ersten Behandlungstagen gesenkt werden, während es nicht zu einer Verminderung der Gesamtabbruchrate gekommen ist (Gößling et al. 1998). Behandlungsabbrüche während der Entgiftungsphase bedingen nach den klinischen Erfahrungen in vielen Fällen eine Verschlimmerung der Suchterkrankung. Außerdem ist bekannt, dass es in der Folge eines Behandlungsabbruchs häufig zu vital bedrohlichen und nicht selten letal endenden Drogennotfällen mit Überdosierungen kommt (Rössler et al. 1993). Unter dem Aspekt einer verminderten Rate von Frühabbrechern bei etwa gleicher Gesamtabbruchrate im Rahmen des methadongestützten Entzugs ist es sehr wichtig, Anstrengungen zu unternehmen, um das nun eröffnete therapeutische Fenster effektiv zu nutzen. Wie zuvor beschrieben, hat es in den letzten Jahren hier bereits verschiedene Ansätze gegeben, um die Behandlungsmotivation der Patienten zu stärken und sie besser auf die sie erwartenden Hürden im Verlaufe ihrer Entgiftungsbehandlung vorzubereiten (motivationale Gesprächsführung - Berman et al. 2010; Skillstraining - Lüdecke et al. 2010; Stuppe 2002). Die vorliegende Arbeit ist in diese Bemühungen einzureihen.

Frühabbrüche in den ersten Behandlungstagen kamen in Übereinstimmung mit früheren Befunden auch in der vorliegenden Studie nur in sehr wenigen Fällen vor (siehe auch Tabelle 2, S. 70).

Betrachtet man die Ergebnisse, so wird deutlich, dass kein Unterschied in der Abbruchrate zwischen den beiden Untersuchungsgruppen zu finden ist. Sowohl in der Kontroll- als auch in der Experimentalgruppe lag die Abbruchquote bei $45 \%$. Damit deckt sich die gefundene Abbruchquote mit den Ergebnissen zahlreicher anderer Autoren (siehe auch Tabelle 1, S. 4). Die Hypothese, dass der eingesetz- 
te videobasierte Furchtappell das Durchhaltevermögen in der Behandlung verbessert, ließ sich also nicht generell bestätigen, da die Abbruchquoten in der Kontrollgruppe und der Experimentalgruppe mit Videodokumentation identisch waren.

Für die komplette Durchführung des im Studiendesign angestrebten videobasierten Furchtappells bei der Experimentalgruppe (1. Videodokumentation der Aufnahmesituation und 2. Videokonfrontation am sog. Nulltag) ließen sich 2/3 der Patienten der Experimentalgruppe gewinnen. Da es sich um eine Feldstudie bei drogenabhängigen Patienten handelt, ist angesichts dieser schwierigen Klientel die Teilnahme von 2/3 der Probanden als gutes Ergebnis zu bewerten und entspricht sicher auch dem, was im klinischen Altag erreichbar wäre. Die Tatsache, dass nicht alle Patienten das Video tatsächlich ansahen, beeinträchtigt wahrscheinlich die Möglichkeit, ein verbessertes Durchhaltevermögen durch den Furchtappell aufzuzeigen. Die Videokonfrontation stellt einen zentralen Bestandteil des intendierten Furchtappells dar. Aus ethischen Gründen ist es aber natürlich nicht möglich, den Patienten das Video entgegen ihrem Willen zu zeigen. Ein solches Vorgehen würde darüber hinaus möglicherweise auch eine ungünstige innere Verarbeitung der persuasiven Botschaft mit sich bringen.

Doch kann man eine Aussage darüber treffen, welche Personen einer Videokonfrontation auch tatsächlich zustimmten? Vergleicht man diese Gruppe mit der Kontrollgruppe ohne Videodokumentation hinsichtlich ihres Durchhaltevermögens, dann findet man in der Experimentalgruppe eine signifikant geringere Abbruchquote $(22 \%$ vs. $45 \%)$. Dabei bleibt offen, inwiefern das bessere Durchhaltevermögen dieser Untergruppe durch die Videokonfrontation beeinflusst ist. Denkbar ist, dass die Probanden dieser Subgruppe von vorn herein besonders motiviert waren, die Entgiftungsbehandlung durchzuhalten und daher auch der Videokonfrontation zustimmten, um sich unterstützen zu lassen. So könnte es sein, dass die Rezipienten aufgrund ihrer besonders guten Behandlungsmotivation auch unabhängig von einer Videokonfrontation die Behandlung regulär abgeschlossen hätten.

Diese Hypothese liegt nahe, da auffällt, dass in der Subgruppe, die die Videokonfrontation ablehnte, von 19 Patienten 18 die Behandlung abbrachen, wobei 17 den Nulltag erst gar nicht erreichten. Dagegen war es in der Subgruppe mit Videodokumentation und -konfrontation so, dass von 41 Probanden nur 9 die Behandlung 
abbrachen und 38 von den 41 Rezipienten den Nulltag erreichten. Die 3, die den Nulltag nicht erreichten, schauten ihr Video aufgrund ihres Abbruchwunsches vor dem Nulltag an, was jedoch nicht dazu führte, dass sie ihre Entscheidung zum Abbruch zurücknahmen.

Insgesamt betrachtet konnte empirisch kein Zusammenhang zwischen Durchhaltevermögen und Anwendung des Furchtappells belegt werden. Es wurde aber ein anderer Aspekt sehr deutlich: Eine besondere Problematik ergibt sich bei jenen Patienten, die den Nulltag erst gar nicht erreichen. Diese waren auch durch andere therapeutische Angebote (therapeutisches Gespräch, Reservemedikation, Akupunktur, Änderung des Behandlungsziels im Sinne der Einstellung auf ein Substitut etc.) nicht von ihrem Vorhaben abzubringen, die Behandlung vorzeitig zu beenden. Es wird wichtig sein herauszufinden, welche Faktoren hier prognostischen Wert haben, und ob es Methoden gibt, präventiv die Behandlungsmotivation gerade dieser Patienten zu stärken.

\subsection{Diskussion zum Zusammenhang zwischen innerer Verarbeitung des Furchtappells und dem Durchhalte- vermögen bzw. Abbruchverhalten im stationären Dro- genentzug}

Um die innere Verarbeitung des Furchtappells und dessen Einfluss auf das Durchhaltevermögen im stationären Drogenentzug genauer zu erfassen, wurde in der vorliegenden Studie von den Rezipienten ein eigens dazu konzipierter Fragebogen direkt nach Ansicht des Videos beantwortet. Darin wurde unter anderem die aversive gefühlsmäßige Berührung erfasst. Die Furchtstärke wurde nicht bewusst experimentell variiert, daher war eine genaue Auswertung nach Subgruppen unterschiedlicher Furchtstärke nicht möglich. Vor dem Hintergrund unserer klinischen Erfahrungen wurde in der vorliegenden Untersuchung die Auslösung einer mäßigaversiven emotionalen Reaktion dem fortgesetzten Drogenkonsum gegenüber angestrebt. Im therapeutischen Gespräch direkt im Anschluss an die Videokonfrontation war es z.B. möglich - wie bereits beschrieben - übermäßige induzierte 
Scham zu reduzieren. Bei der Darstellung der inneren Verarbeitung in Tabelle 5 (S. 79) wird deutlich, dass der Mittelwert für die gefühlsmäßige Berührung bei 0,9671 (Min. 0; Max. 2; S. 0,58751) liegt. Es ist jedoch nicht quantitativ belegbar, inwiefern eine mäßige Aktivierung aversiver emotionaler Schemata im Einzelfall tatsächlich geglückt ist.

\section{Aversive gefühlsmäßige Berührung}

In der vorliegenden Arbeit stellte sich heraus, dass entgegen der Untersuchungshypothese stärkere aversive gefühlsmäßige Berührung bis zu einem mittleren Ausmaß nicht mit einem besseren Durchhaltevermögen in der Entgiftungsbehandlung einhergeht. Dieser Befund unterstützt die These von Gößling et al. (2001), dass bei Suchtpatienten die bewusste Wahrnehmung eines schlechten Gesundheitszustandes nicht als Antriebsfeder für das Durchhaltevermögen während einer Entgiftungsbehandlung fungiert, scheint aber den Ergebnissen der Furchtappellforschung zu widersprechen, nach denen die Furchtinduktion auch in der Gesundheitsprävention eine erfolgreiche Technik zur Einstellungs- und Verhaltensänderung darstellt (Barth und Bengel 1998).

Dieses Ergebnis könnte darauf hinweisen, dass eine Induktion von Furcht mittleren Ausmaßes nicht geglückt ist. Die Probanden waren eventuell zu wenig aversiv aktiviert. Eventuell handelt es sich hier aber auch um ein komplexes Zusammenspiel so unterschiedlicher Faktoren, dass ein einfacher Zusammenhang nicht aufzeigbar ist. So ist möglicherweise dieses Ergebnis mit darauf zurückzuführen, dass nach neueren Theorien zur Wirkung von Furchtappellen nicht nur die Höhe der induzierten Furcht, sondern auch das ausgelöste Selbstwerterleben und das Ausmaß angebotener Bewältigungsstrategien wichtige Einflussfaktoren auf das Ausmaß der Einstellungsänderung darstellen.

Sowohl in den Untersuchungen zum Videofeedback (Baker et al. 1975; Eisler et al. 1973; Harvey et al. 2000; Kim et al. 2002, Rapee und Hayman 1996), als auch in der Furchtappellforschung erwiesen sich therapeutische Instruktionen als wesentliche Bestandteile der therapeutischen Wirksamkeit. Demnach ist die Furchtinduktion zwar als motivationaler Faktor für eine Verhaltensänderung sinnvoll, sie sollte jedoch auf jeden Fall mit der Vermittlung von Bewältigungsmöglichkeiten 
gekoppelt sein (Leventhal 1970; Rogers 1975,1983). Diese Erkenntnisse wurden in der vorliegenden Untersuchung berücksichtigt - die Probanden wurden einerseits kognitiv auf den videobasierten Furchtappell vorbereitet, andererseits hatten sie ein detailliertes therapeutisches Gespräch im Anschluss an die Feedbacksitzung. Ein Furchtappell bedroht das bereits zuvor niedrige Selbstwertgefühl der Patienten zusätzlich. Es war daher hier besonders wichtig, durch das therapeutische Gespräch diese Bedrohung wieder aufzufangen und konkrete Bewältigungsstrategien zu vermitteln. Dies scheint auch geglückt zu sein, denn diejenigen, die das Video sahen, hatten - wie beschrieben - auch die geringere Abbruchquote. Bei denjenigen, die die Konfrontation ablehnten, war möglicherweise die Bedrohung des Selbstwertgefühls schon im Vorfeld zu massiv; diese Probanden konnten jede weitere Selbstwertbedrohung durch den Videoinhalt nicht tolerieren.

Es stellt sich des Weiteren die Frage, ob die Konzipierung des Fragebogens ausreichend geglückt ist (vergl. S. 101, Gesamtbetrachtung zur inneren Verarbeitung). Zudem ist zu überdenken, zu welchem Zeitpunkt ein Fragebogen zur inneren Verarbeitung des videobasierten Furchtappells von den Rezipienten am besten beantwortet wird: Direkt nach Ansicht des Videos, wie es in der vorliegenden Untersuchung geschehen ist, oder besser im Anschluss an das begleitende therapeutische Gespräch, das ja offensichtlich einen zentralen Wirkfaktor darstellt. Möglicherweise wäre es auch sinnvoll, den Rezipienten vor Beantwortung des Fragebogens einen oder mehrere Tage Zeit zur Reflexion und Verarbeitung der Videofeedbacksitzung zu lassen; zumal innen manche Gedankengänge eventuell auch erst mit dem Nachlassen von Entzugssymptomen und Suchtdruck möglich sind.

\section{Induzierter Suchtdruck}

Neben der aversiven Reaktion auf das Video wurde danach gefragt, ob durch die Ansicht des Videos zusätzlicher Suchtdruck ausgelöst wurde. Die Videokonfrontation erzeugte bei den meisten Probanden keinen zusätzlichen Suchtdruck. Auch dieser Befund deutet darauf hin, dass die Gestaltung des Furchtappells insoweit geglückt ist, dass keine zu starke aversive Aktivierung erfolgt ist. Daneben war bei denjenigen, die das Video ansahen, ein besseres Durchhaltevermögen aufzeigbar. Es wurde insgesamt kein Zusammenhang zwischen dem Ausmaß induzierten 
Suchtdrucks und dem Durchhaltevermögen gefunden. Es ist allerdings davon auszugehen, dass ein zu starker induzierter Suchtdruck mit einem wahrscheinlicheren Abbruch der Entgiftung einhergeht. Im vorliegenden Fall lässt die geringe Varianz in den Daten es kaum zu, Zusammenhänge auf statistischer Ebene aufzudecken.

Für zukünftige Studien ist anzumerken, dass das Ausmaß des induzierten Suchtdrucks durch das Item 4 des Fragebogens nicht optimal erfasst wird. Günstiger erscheint es, vor bzw. nach Ansicht des Videos eine visuelle Analogskala zu nutzen, um den absoluten Wert des Suchtdrucks zu bestimmen. Die Differenz der beiden erhobenen Werte könnte dann genutzt werden, um den induzierten Suchtdruck und seinen Zusammenhang zum Durchhaltevermögen zu bestimmen.

\section{Reaktanz der Probanden}

Der bei der Auswertung gefundene dritte Faktor: „Das Video berührt gefühlsmäßig nicht", wurde als Reaktanz interpretiert. Nach Brehm (1966) ist mit psychologischer Reaktanz eine komplexe Abwehrreaktion gegen eine äußere oder innere Einschränkung gemeint. Dabei bezeichnet man mit Reaktanz im eigentlichen Sinne nicht das ausgelöste Verhalten, sondern die zugrunde liegende Haltung.

In der vorliegenden Studie erschien uns einerseits nicht plausibel, dass keinerlei gefühlsmäßige Reaktion durch das Video ausgelöst worden sein soll. Es wurden andererseits auch Beobachtungen aus der Phase der Erhebung genutzt: Die Probanden brachten zum Teil auch sehr offensichtlich verbal und nonverbal inre Abwehr dem Videofeedback gegenüber zum Ausdruck. Manche Patienten hatten schon in der Aufnahmesituation eine ablehnende Haltung gegenüber der Studie und stimmten einer Teilnahme erst gar nicht zu. Es wäre interessant zu wissen, wie die Abbruchquote dieser Patienten aussieht. Andere stimmten zwar der Videodokumentation zu, lehnten aber die Videokonfrontation ab, noch andere begannen zwar die Feedbacksitzung, brachen diese jedoch im Verlauf ab, und schließlich mag es auch Probanden gegeben haben, deren Reaktanz sich vor allem gegen die Beantwortung des Fragebogens gerichtet haben mag. Ob die erfasste Reaktanz sich mehr auf die Videokonfrontation, die Beantwortung des Fra- 
gebogens oder vielleicht auch auf das noch anstehende therapeutische Gespräch bezog, bleibt unklar.

Insgesamt ist zwischen der erfassten Reaktanz und der Abbruchhäufigkeit kein Zusammenhang erkennbar. Dies belegt möglicherweise die Hypothese, dass das bessere Durchhaltevermögen der Patienten mit Videokonfrontation kein spezifischer Effekt des videobasierten Furchtappellls ist, sondern dass es sich bei der Subgruppe mit Videokonfrontation um Patienten handeln könnte, die die Entgiftung auch unabhängig von der Viedeokonfrontation besser abgeschlossen hätten als der Durchschnitt (siehe auch Kapitel 4.2, S. 94 f.).

\section{Gesamtbetrachtung zur inneren Verarbeitung}

Offensichtlich steht das bessere Durchhaltevermögen der Patienten in der Experimentalgruppe mit Videodokumentation und Videokonfrontation also weder in einem nachweisbaren Zusammenhang mit der aversiven Reaktion auf das Video noch mit deren Ausmaß an Reaktanz, noch mit etwaig zusätzlich ausgelöstem Suchtdruck.

Demnach muss das Durchhaltevermögen im stationären Drogenentzug mit einem anderen nicht erfassten Wirkfaktor in Zusammenhang stehen. Dabei bleibt offen, ob dieser Faktor vom Furchtappell abhängig ist oder nicht.

Möglicherweise werden in dem Fragebogen die Gefühle, Kognitionen und Handlungstendenzen, die das Video bei den Probanden hinsichtlich des Durchhaltevermögens in der Entgiftungsbehandlung induziert, nicht hinreichend erfasst. Diese Vermutung liegt nahe, da in der Gruppe mit Videokonfrontation zum einen eine signifikant geringere Abbruchquote vorhanden ist als in einer gleich großen Kontrollgruppe. Zum anderen antwortete die Mehrzahl der Rezipienten mit „ja“ bei der Frage danach, ob die Videodokumentation und -konfrontation bei allen Patienten durchgeführt werden sollte. Daraus könnte man schließen, dass die Anwendung der Videokonfrontation als hilfreich erlebt wird. Einschränkend ist anzumerken, dass die Rezipienten vielleicht auch aus Überangepasstheit mit „ja“ auf die o.g. Frage antworteten und der Furchtappell tatsächlich keine Relevanz im Hinblick auf die Abbruchquote hat. 
Ein wichtiger Gesichtspunkt ist die Konstruktion des Fragebogens. Es wäre günstiger gewesen, die Voraberhebung ausführlicher zu gestalten, da das Augenmerk dann frühzeitiger auf den Aspekt der Reaktanz gelenkt worden wäre. Es gilt nun den Fragebogen eingehend zu überarbeiten: Augenscheinvalidität ist gegeben. Andere Validitätsmaße zu finden erweist sich in diesem speziellen Fall als schwierig, physiologische Parameter beispielsweise sind unbrauchbar aufgrund der akuten Entzugssymptomatik. Alternative Verfahren zum Fragebogen existieren nicht. Schließlich sollte auch genauer betrachtet werden, welche Items die jeweilige Skala optimal erfassen.

Es könnte sein, dass bei den Rezipienten durch die Teilnahme an der Furchtappelluntersuchung eine besondere Behandlungsmotivation gewachsen ist, welche innen eher einen erfolgreichen Behandlungsabschluss ermöglichte. In diesem Sinne könnte es sein, dass das durch die Studienteilnahme bekundete Interesse am Schicksal der Probanden und die zusätzlich erlebte Zuwendung sich positiv auf die Behandlungsmotivation und Durchhaltefähigkeit auswirkten, indem sie z. B. die therapeutische Beziehung positiv beeinflussten. In verschiedenen Untersuchungen wurde nachgewiesen, dass interaktionelle Prozesse, persönliche Einstellung des Patienten und subjektive Vorerwartungen einen maßgeblichen Einfluss auf den Entzugsverlauf ausüben (Gößling et al. 2001, Hirsch und Tretter 2000). Des Weiteren ist bekannt, dass Drogen-Patienten ein besonders schwierig zu behandelndes Patientenkollektiv darstellen, in deren Behandlung eine tragfähige therapeutische Beziehung ausgesprochen wichtig ist (Liese und Beck 1997; Meier et al. 2005 a; Meier et al. 2005 b). Die Teilnahme an der Furchtappelluntersuchung mittels Videodokumentation und Videokonfrontation beeinflusst möglicherweise gerade diese für entscheidend gehaltenen Faktoren nämlich: interaktionelle Prozesse, persönliche Einstellung des Patienten und subjektive Verantwortung sowie die Art der Arzt-Patientenbeziehung günstig.

Darüber hinaus ist es nicht unwahrscheinlich, dass für unterschiedliche Individuen verschiedene, individuelle Wirkfaktoren hinsichtlich eines erfolgreichen Behandlungsverlaufs existieren, die man nur durch gezielte Befragung der einzelnen Personen ermitteln könnte. 
Auf individuelle Faktoren (wie z.B. Intelligenzniveau, Selbstbild, Sympathieempfinden für den Sender der Botschaft...), die die Verarbeitung einer präventiven Botschaft und damit das Ausmaß und die Richtung der erzielten Einstellungs- und vor allem der Verhaltensänderung beeinflussen, weisen schon Petty et al. (1991) hin (siehe auch Abbildung 5, S. 36).

Nach der klinischen Erfahrung bilden drogenabhängigen Menschen eine spezielle Klientel, die in der Therapie ein besonderes auf die spezifischen Bedürfnisse dieser Menschen und deren Besonderheiten abgestimmtes Vorgehen benötigt. Zusätzlich handelt es sich um eine heterogene Gruppe (Geschlecht, Art der Suchtmittel, mono- oder polyvalenter Konsum, Krankheitsdauer, individuelle Einstellungen zum Suchtverhalten bzw. zur Abstinenz, unterschiedliche Selbstbilder, etc.). Möglicherweise bedarf es einerseits eines zielgruppenspezifischen Furchtappells mit andererseits individueller Nachbereitung desselben im therapeutischen Gespräch, wie es in der vorliegenden Studie geschehen ist. Dabei eröffnet sich ein weites Feld zur Erforschung individueller Wirkfaktoren in der Therapie suchtkranker Menschen, um das therapeutische Angebot zu optimieren und die Effizienz der Behandlung zu verbessern.

In einem Einzelfall wurden kulturelle Aspekte bei der Wirkung des FurchtappellVideos deutlich. Ein Proband deutschrussischer Abstammung empfand das Ansehen des Videos als hochgradig peinlich, brach die Konfrontation daraufhin sogar ab. Dennoch beendete er die Entgiftungsbehandlung erfolgreich, obwohl davon auszugehen ist, dass bei ihm ein sehr hohes $\mathrm{Ma} ß$ an induzierter aversiver Aktivierung bestand. Dieser Einzelfall lenkt die Aufmerksamkeit auf einen Gesichtspunkt, der bislang noch keine Berücksichtigung fand: Es erscheint wichtig, bei der praktischen Anwendung des Furchtappells im Rahmen einer stationären Entgiftung auch kulturelle Besonderheiten zu berücksichtigen und in folgenden empirischen Untersuchungen näher zu betrachten. 


\subsection{Diskussion zur geschlechtsspezifischen Wirkung von Furchtappellen}

Nach den Literaturangaben sind substanzabhängige Frauen weniger als Männer dazu bereit, sich einer Therapie ihrer Substanzabhängigkeit zu unterziehen. Sie neigen außerdem eher dazu, begonnene Therapieversuche abzubrechen (Petry und Bickel 2000; Gößling et al. 2001). Dieses konnte in der vorliegenden Arbeit nicht bestätigt werden. Weder in der Experimentalgruppe noch in der Gruppe ohne Videokonfrontation war ein Zusammenhang zwischen Geschlecht und Abbruch feststellbar. Ob bei einer größeren Stichprobe ein diesbezüglicher Effekt bei den Frauen aufgetreten wäre, bleibt offen.

In der vorliegenden Studie, die nach Nasseri (1979) auch als Studie zur Rückfallprävention gelten kann, ergab sich kein Unterschied hinsichtlich der Bereitschaft von Männern und Frauen sich das Video auch tatsächlich anzusehen. Lairson und Swint (1978) dagegen zeigten, dass Frauen Maßnahmen der Gesundheitsprävention gegenüber eher zugänglich sind als Männer.

Frauen wurden wie erwartet durch das Video stärker aversiv gefühlsmäßig berührt als die betroffenen Männer. Die Frauen der Experimentalgruppe mit Videokonfrontation entwickelten tendenziell einen stärkeren durch die Ansicht des Videos induzierten Suchtdruck und sie reagierten auf das Video weniger reaktant als die männlichen Rezipienten. Letzteres Ergebnis befindet sich in Übereinstimmung mit den geschlechtsspezifischen Unterschieden bei Alkoholikern, bei denen Männer die größte Reaktanz und größere Intention zu trinken nach Aufforderung zur Abstinenz zeigten (Southwick-Bensley und Wu 1991). Allerdings war in der vorliegenden Studie kein Zusammenhang zwischen dem Ausmaß an Reaktanz bzw. Suchtdruck und einem Therapieabbruch nachweisbar. Es zeigte sich aber, dass die Frauen, die der Experimentalgruppe mit Videokonfrontation angehörten tendenziell eher abbrachen als die Männer der Subgruppe mit Videokonfrontation. Dieses Ergebnis befindet sich im Gegensatz zu den Befunden von SouthwickBensley und Wu (1991), in deren Studie die männlichen Alkoholiker nach Aufforderung zur Abstinenz eine größere Reaktanz und Intention zu Trinken zeigten! Angesichts der in der vorliegenden Arbeit gefundenen Ergebnisse ist es um so 
überraschender, dass Frauen eher als Männer dafür sind, das Vorgehen mittels Videodokumentation und -konfrontation bei allen Patienten in der Entgiftungsbehandlung durchzuführen, zumal bei den Frauen der Experimentalgruppe mit Videokonfrontation kaum eine Verbesserung ihres Durchhaltevermögens in der Entgiftungsbehandlung gegenüber der Kontrollgruppe ( $40 \%$ vs. $45 \%)$ feststellbar ist. Möglicherweise schätzen die Frauen zwar sehr wohl das innen durch die Videodokumentation und -konfrontation entgegengebrachte Interesse. Und obwohl sie dadurch emotional stärker berührt werden als die männlichen Rezipienten (mehr Reaktanz bei den Männern) und somit den Hypothesen von Leventhal (1970) und Rogers (1975) zufolge eher von dem videobasierten Furchtappell profitieren müssten, trifft dies nicht zu.

Zwar induzierte die Ansicht des Videos tendenziell einen stärkeren Suchtdruck bei den Frauen als bei den Männern. Insgesamt führte die Ansicht des Videos aber zu keiner zusätzlichen signifikanten Suchtdruckbelastung führte. Es war auch kein Zusammenhang zu einem Behandlungsabbruch erkennbar. Die Ergebnisse einer höheren Suchtdruckbelastung bei Frauen gegenüber Männern befinden sich in Übereinstimmung mit Ergebnissen von Elman et al. (2001). Die Autoren konnten bei kokainabhängigen Frauen im Vergleich zu Männern signifikant höhere Craving scores nachweisen. Sie stellten zudem ein größeres Ausmaß depressiver Symptome und eine höhere Belastung mit sozialen und familiären Problemen bei den betroffenen Frauen fest, brachten diese jedoch nicht mit den erhöhten Craving scores in Verbindung. Sie vermuten vielmehr einen über das dopaminerge System vermittelten Zusammenhang zwischen erhöhtem Craving und weiblichen Geschlechtshormonen.

Das Unvermögen der Frauen der Experimentalgruppe eine Verbesserung ihres Durchhaltevermögens in der Entgiftungsbehandlung gegenüber der Kontrollgruppe zu erzielen, könnte möglicherweise mit einer deutlich höheren Belastung drogenabhängiger Frauen mit psychiatrischen Begleiterkrankungen zusammenhängen.

Die psychiatrischen Begleiterkrankungen wurden zwar in der vorliegenden Untersuchung nicht diagnostiziert, da zu Beginn einer Entgiftungsbehandlung häufig Intoxikationen/ Mischintoxikationen oder auch Entzugssyndrome bestehen. Letzte- 
re dominieren den psychopathologischen Zustand der Probanden auch in der Endphase der Entgiftungsbehandlung, so dass insgesamt aufgrund der Überlappung der Syndrome einerseits sowie der Kürze der Behandlungsdauer einer dreivierwöchigen stationären Entgiftung andererseits eine zu hohe Wahrscheinlichkeit von Fehldiagnosen resultiert (siehe auch Kapitel 1.1.1).

Es ist jedoch bekannt, dass bei opiatabhängigen Frauen etwa zweimal so häufig eine weitere psychiatrische Diagnose gestellt wird wie bei opiatabhängigen Männern. Opiatabhängige Frauen haben auch häufiger eine zusätzliche dritte psychiatrische Diagnose nach ICD-10 (69\% der Frauen gegenüber 49\% der Männer) (Günther et al. 2000). Bei der Lebenszeitprävalenz lagen die Werte der drogenabhängigen Frauen bei den Angst- und Zwangsstörungen sowie den affektiven Störungen mit jeweils 55\% höher als bei den Männern (49\% bzw. 37\%) (Günther et al. 2000). Außerdem zeigen drogenabhängige Frauen mehr suizidales Verhalten als drogenabhängige Männer (Cottler et al. 2005). Auch traumatisierende Kindheitserfahrungen erhöhen das Risiko für einen Suizidversuch um das 2-5-fache (Dube et. al. 2001) und stellen einen Risikofaktor für die spätere Entwicklung einer Angststörung oder Depression (Safren et al. 2002; Engel et al. 1993; Bremner et al. 1993 a, b) sowie Essstörung (Newton-Taylor et al. 1998) dar. Kessler et al. (1995) fanden eine Lebenszeitprävalenz für die PTBS von 7,8\%, dabei waren Frauen mehr als doppelt so häufig betroffen wie Männer (10,4\% vs. 5,0\%). Das Risiko für die Entwicklung einer Substanzabhängigkeit ist nach den Ergebnissen der Epidemiologic-Catchment-Area-Studie (Burnam et al. 1988) bei Frauen nach sexuellem Missbrauch erhöht. McCauley et al. (1997) berichten bei kindlicher Traumatisierung durch körperliche Gewalt oder sexuellen Missbrauch über eine 4,7-fach erhöhte Prävalenz von Drogenmissbrauch, eine 2,2-fach höhere Prävalenz von Alkoholabusus, 3,7-fach mehr Suizidversuche und ein 3,2-fach erhöhtes Risiko psychiatrischer Hospitalisierungen. Kreyssig (1997) beziffert den Anteil sexuell missbrauchter Frauen an allen drogensüchtigen Frauen in Deutschland auf $70 \%$. Krausz und Briken (2002) fanden bei opiatabhängigen Frauen einen Anteil von $41,3 \%$ mit sexuellen Missbrauchserfahrungen. Einen sexuellen Missbrauch vor dem 14. Lebensjahr stellten Teegen und Zumbeck (2000) bei $38,5 \%$ der opiatabhängigen Frauen und 4,2\% der opiatabhängigen Männer fest. In einer Untersu- 
chung von Schäfer et al. (2000) gaben 70\% der Frauen und 56\% der Männer mit polyvalentem Substanzgebrauch bei einer weiten Missbrauchsdefinition an vor dem 16. Lebensjahr sexuell missbraucht worden zu sein.

Als Problem in der Behandlung von Patienten mit komplexen Traumafolgestörungen beschreibt Wöller (2006) umfassende Störungen der Emotionsregulierung. Diese sind dabei gekennzeichnet durch den Wechsel unterschiedlicher emotionaler Zustände. Außerdem sind die betroffenen Patienten nur eingeschränkt in der Lage, die Intensität ihrer Affekte zu regulieren und geraten nicht selten in Zustände, in denen sie sich von Affekten überflutet fühlen. Meist ist auch die Fähigkeit reduziert, die Aufmerksamkeit zu fokussieren und die Impulskontrolle ist vermindert. Oft können die Gefühle nicht differenziert erfasst werden; es werden statt dessen Zustände unerträglicher diffuser Spannungen erlebt. Neben Depressivität, Gefühlen von Leere Angst, Wut, Scham- und Schuldgefühlen stehen typischerweise Gefühle des Alleingelassenseins sowie Gefühle umfassender Hilflosigkeit und ohnmächtigen Ausgeliefertseins im Vordergrund.

Bei einer Entgiftungsbehandlung geraten drogenabhängige Patienten im Rahmen eines Entzugssyndroms nicht selten in Zustände, die mit ähnlichen Schwierigkeiten verbunden sind, wie die umfassenden Folgestörungen komplexer Traumatisierungen und die sich bei Komorbidität einer Suchterkrankung und PTBS potenzieren.

Eine tendenziell stärkere affektive Berührung sowie die geringere Reaktanz hinsichtlich der inneren Verarbeitung des videobasierten Furchtappells bei den Frauen hängt möglicherweise mit deren stärkerer Belastung mit Traumatisierungen und posttraumatischen Belastungsstörungen zusammen. Damit ist auch die oben beschriebene Störung der Affektverarbeitung verbunden sowie eine reduzierte Fähigkeit, die Aufmerksamkeit zu fokussieren und Impulse zu kontrollieren. Dies könnte dazu führen, dass eine Videokonfrontation am Nulltag zwar affektive Mobilisierung hervorruft, jedoch infolge der Tatsache, dass nun psychiatrische Begleiterkrankungen nicht mehr durch etwaige Drogenwirkungen kupiert werden und sich statt dessen mit den entzugsassoziierten psychischen und körperlichen Problemen potenzieren (siehe auch Kapitel 1.1.3), nicht mehr in für die Behandlung fruchtbares Denken und Handeln umgesetzt werden kann. Für die Gruppe dro- 
genabhängiger Frauen bedarf es somit auch in der Entgiftungsbehandlung der Berücksichtigung ihrer Besonderheiten: höherer Suchtdruck, größere Belastung mit komorbiden psychiatrischen Störungen einschließlich Suizidalität, darunter auch eine höhere Belastung mit Traumatisierungen und Posttraumatischen Belastungsstörungen inklusive der damit verbundenen Störungen der Affektregulation. Für den Einsatz eines videobasierten Furchtappells wäre beispielsweise zu klären, ob Frauen in der Drogenentgiftung eher davon profitieren, wenn die Videokonfrontation nicht am sog. Nulltag stattfindet, sondern zu einem späteren Zeitpunkt, wenn die Affektlage ausgeglichener ist.

Wöller (2006) zeigt verschiedene, spezielle Behandlungsansätze auf, die sich des Problems der Affektregulationsstörung bei traumatisierten Patienten annehmen, da die betroffenen Patienten von den herkömmlichen Therapieangeboten krankheitsbedingt häufig nicht profitieren können. Es ist zu überlegen, welche dieser Behandlungsansätze sich mit den Konzepten einer Entgiftungsbehandlung verknüpfen lassen, um in Zukunft ein zielgruppenspezifisches Angebot vorhalten zu können.

Es ist allgemein bekannt, dass suchtkranke Patienten mit komorbiden psychiatrischen Störungen mehr Behandlungsschwierigkeiten, einen erhöhten Substanzkonsum und stärkere soziale Belastungen gegenüber ihren Mitpatienten ohne psychiatrische Zusatzdiagnosen haben. Das Vorliegen einer komorbiden psychiatrischen Erkrankung bei Drogenabhängigen (Rounsaville et al. 1986) ist im Allgemeinen mit einer schlechteren Behandlungsprognose verbunden. Ausschlaggebend für den Behandlungsverlauf scheint dabei die Symptombelastung zu sein (McLellan et al. 1983).

Schnieders et al. (2006) untersuchten 100 drogenabhängige Probanden mittels eines Traumafragebogens, wobei sexuelle und körperliche Missbrauchserfahrungen, Vernachlässigung, familiäre Gewalt und emotionale Misshandlung erfasst wurden. Sie fanden für die untersuchte Studienpopulation sowohl für das körperliche als auch für das psychische Befinden eine deutlich höhere Symptombelastung als die Referenzgruppe ohne sexuelle Missbrauchserfahrungen. Die Analyse bezüglich des Einflusses geschlechtsspezifischer Unterschiede wies für die Skalen 
Unsicherheit im Sozialkontakt und phobische Angst signifikant höhere Werte für Frauen auf.

Die Symptome einer PTBS, Angststörung oder Depression sind nach unseren klinischen Erfahrungen bei Opiatabhängigen bzw. Patienten mit polyvalentem Substanzkonsum inklusive einer Opioidabhängigkeit häufig durch die Suchterkrankung kupiert. Es besteht aber die Gefahr, dass sie im Rahmen einer Entgiftungsbehandlung exazerbieren, wobei nicht selten suizidale Tendenzen auftreten. Es ist bekannt, dass substanzabhängige Frauen eher dazu neigen, begonnene Therapieversuche abzubrechen (Backmund et al. 2001; Petry und Bickel 2000) bzw., dass sie generell weniger dazu bereit sind, sich einer Therapie ihrer Substanzabhängigkeit zu unterziehen (Green 2006). Die Scheu eine Therapie der Suchterkrankung anzugehen und auch der Abbruch einer Entgiftungsbehandlung bei Patienten, die mit einer oder mehreren zusätzlichen psychiatrischen Erkrankungen belastet sind - was häufiger auf Frauen zutrifft - ist möglicherweise auch einen Versuch, drohender weiterer psychischer und bzw. auch suizidaler Dekompensation entgegenzuwirken und stellt aus dieser Perspektive auch einen Akt der Selbstfürsorge dar. 
In der vorliegenden Arbeit wurde der Fragestellung nachgegangen, ob das Durchhaltevermögen im stationären Drogenentzug mittels eines videobasierten Furchtappells verbessert werden kann. Der Untersuchung wurde eine randomisierte, hinsichtlich des Geschlechts geschichtete Strichprobe von insgesamt 120 Probanden zugrunde gelegt. Dabei handelte es sich um Patienten, welche zwischen Mai 2004 und Dezember 2005 zur Entgiftung illegaler Drogen im ehemaligen Niedersächsischen Landeskrankenhaus Göttingen auf einer Drogenentzugsstation aufgenommen worden waren und bei denen nach den diagnostischen Kriterien der ICD -10 eine Opioidabhängigkeit bzw. eine Polytoxikomanie inkl. einer Opioidabhängigkeit bestand.

Es wurde geprüft, ob sich das Durchhaltevermögen im Entzug dadurch erhöht, dass der Hälfte der Probanden am sog. „Nulltag“8 ihrer Entgiftung das von innen am Tag der Krankenhausaufnahme gemachte Video gezeigt und besprochen wurde.

Hinsichtlich der Abbruchrate bestand kein Unterschied zwischen beiden Gruppen. Sowohl in der Kontrollgruppe als auch in der Experimentalgruppe betrug die Abbruchrate $45 \%$. Die Videodokumentation und -konfrontation als Furchtappell verbessert also nicht generell das Durchhaltevermögen im stationären Drogenentzug. Allerdings stimmten nur gut 2/3 der Probanden der Experimentalgruppe einer Videokonfrontation auch tatsächlich zu. Dabei ist bemerkenswert, dass in dieser Subgruppe (mit Videokonfrontation) eine signifikant geringere Abbruchhäufigkeit bestand $(24,5 \%$ Abbrecher in der Experimentalgruppe mit Videokonfrontation gegenüber $45 \%$ in der Kontrollgruppe; $p=0,035$ ).

Gegenstand weiterer Untersuchungen bleibt die Frage, ob die Verbesserung des Durchhaltevermögens dieser Patientensubgruppe ein spezifischer Effekt der Videokonfrontation ist oder ob es sich hier um eine ohnehin besonders motivierte Kli-

\footnotetext{
${ }^{8}$ Nulltag heißt der erste Behandlungstag, an dem die zu entgiftende Substanz nicht mehr verabreicht wird. Werden mehrere Substanzen entgiftet so zählt als Nulltag, der erste Tag an dem keine der zu entgiftenden Substanzklassen mehr eingenommen wurde.
} 
entel handelte, die auch ohne diese Maßnahme erfolgreich in der Behandlung gewesen wäre.

Bemerkenswert bleibt, dass etwa 1/3 der „Entgifter“ am Ende nicht bereit waren, die zum Untersuchungsdesign dazugehörige Videokonfrontation auch durchzuführen, welche einen integralen Bestandteil des Furchtappells darstellt. Dabei handelt es sich fast ausschließlich um Therapieabbrecher vor dem Nulltag. Sie sind somit ein besonderes Problem.

Im Rahmen der Untersuchung war außerdem die innere Verarbeitung des Furchtappells von Interesse. Wir fanden aber keinen Zusammenhang zwischen dem Ausmaß der gefühlsmäßigen Berührung durch den Furchtappell und der Abbruchrate im stationären Drogenentzug abzubilden (Korrelation nach Pearson: ,071; $p(1$-seitig) $=, 338)$.

Überraschenderweise erfasste der Fragebogen zur inneren Berührung durch den Furchtappell neben dem Ausmaß der gefühlsmäßigen Berührung auch das Ausmaß bestehender Reaktanz auf Seiten der Probanden. Mit Reaktanz ist dabei eine innere Haltung im Sinne einer komplexen Abwehrreaktion gemeint. Diese tritt als Widerstand gegen eine äußere oder innere Einschränkung (z.B. psychischer Druck, Verbote; hier: der Proband soll das Video anschauen und möglichst die Behandlung nicht abbrechen) auf. Es ließ sich jedoch auch keine statistisch signifikante Korrelation zwischen dem Ausmaß der Reaktanz und dem Durchhaltevermögen in der stationären Entgiftung finden (Korrelation nach Pearson: ,056; p (1seitig) $=, 3695$ ).

Die Videokonfrontation erzeugte bei einem Großteil der Patienten keinen zusätzlichen Suchtdruck. Ein möglicher Zusammenhang zwischen erzeugtem Suchtdruck und Durchhaltevermögen bei der Entgiftung konnte jedoch statistisch nicht nachgewiesen werden (Korrelation nach Pearson: ,191; p (2-seitig) = ,258); möglicherweise allerdings aufgrund der fehlenden Varianz in den Antworten.

Im Rahmen der Studie interessierten des Weiteren Geschlechtsunterschiede. Zwischen Männern und Frauen bestand kein signifikanter Unterschied in der Bereitschaft zur Videokonfrontation $\left(\mathrm{Chi}^{2}=0,616 ; \mathrm{p}=0,432\right)$. 
Statistisch ließ sich allerdings bestätigen, dass Frauen durch den Furchtappell stärker aversiv-gefühlsmäßig berührt werden als Männer (Korrelation nach Pearson: ,333; $\mathrm{p}$ (1-seitig) $=$,044). Außerdem konnte erneut nachgewiesen werden, dass Männer signifikant stärker mit einer reaktanten Haltung reagieren als Frauen (Korrelation nach Pearson: ,316, p (1-seitig) $=$,054). Frauen entwickeln durch das Video induziert tendenziell einen stärkeren Suchtdruck als Männer (Korrelation nach Pearson: ,308, p (1-seitig) = ,063). In der Gruppe mit Videokonfrontation zeigte sich tendenziell ein Zusammenhang zwischen Geschlecht und Abbruch, der in der anderen Gruppe ohne Videokonfrontation so nicht aufzufinden ist. Dabei brechen Frauen tendenziell eher ab als Männer $\left(\mathrm{Chi}^{2}=3,125(b) ; p=0,07\right)$.

Der Großteil der Probanden (86,8 \%) hält es für sinnvoll, den videobasierten Furchtappell bei allen Patienten einer stationären Entgiftungsbehandlung einzusetzen. Frauen würden eher das Video als Standard einsetzen als Männer $\left(\mathrm{Chi}^{2}=\right.$ $3,359, p=0,067)$.

Insgesamt wurde deutlich, dass Patienten, die zur Entgiftung illegaler Drogen in ein Krankenhaus kommen, keine homogene Gruppe darstellen und offensichtlich genauerer Untersuchung bedürfen, um subgruppenspezifische Behandlungsangebote unter Einbeziehung geschlechtsspezifischer Besonderheiten entwickeln zu können. Geschlechtsunterschiede wirken sich auch in der Entgiftungsbehandlung auf den Behandlungserfolg aus und erfordern anscheinend ein spezielles Behandlungsangebot. Die Erweiterung des therapeutischen Angebots auf einer Drogenentgiftungsstation um einen videobasierten Furchtappell ist möglicherweise für eine Subgruppe von Nutzen und hat sich für alle Probanden als unschädlich erwiesen. Weitere Anwendung und Erforschung der Methode sind jedoch nötig, um das therapeutische Angebot im stationären Drogenentzug zu optimieren. 


\section{6}

Anhang

\subsection{Anhang A \\ Tabellen: $\mathbf{2 0 - 2 7}$}

Einbezogen wurden 8 Items (siehe auch Abbildung 8 Seite 57), da diese jeweils auf einer Intervallskala beantwortet wurden.

Korrelationsmatrix: Stellt die jeweiligen Interkorrelationen der verschiedenen Variablen dar.

Tabelle 20: Korrelationsmatrix

\begin{tabular}{|l|l|c|c|c|c|c|c|c|c|}
\hline & & Frage1 & Frage2 & Frage3 & Frage4 & Frage5 & Frage6 & Frage7 & Frage8 \\
\hline \multirow{5}{*}{ Kor- } & Frage1 & 1,000 &,- 341 &, 424 &, 117 &, 490 &, 389 &, 514 &,- 461 \\
& Frage2 &,- 341 & 1,000 &, 146 &,- 107 &, 024 &,- 379 &,- 020 &, 335 \\
rela- & Frage3 &, 424 &, 146 & 1,000 &, 039 &, 733 &, 162 &, 441 &,- 286 \\
tion & Frage4 &, 117 &,- 107 &, 039 & 1,000 &, 099 &, 059 &, 148 &, 004 \\
& Frage5 &, 490 &, 024 &, 733 &, 099 & 1,000 &, 151 &, 535 &,- 243 \\
& Frage6 &, 389 &,- 379 &, 162 &, 059 &, 151 & 1,000 &,- 004 &,- 460 \\
& Frage7 &, 514 &,- 020 &, 441 &, 148 &, 535 &,- 004 & 1,000 &, 011 \\
& Frage8 &,- 461 &, 335 &,- 286 &, 004 &,- 243 &,- 460 &, 011 & 1,000 \\
\hline
\end{tabular}

Wichtiges Gütekriterium für FA: Die Kommunalitäten $\mathrm{h}^{2}$

Diese Kommunalitäten sollten jeweils mindestens .50 betragen. Wenn die Kommunalität einer Variablen kleiner ist, dann sollte man sie lieber aus der Analyse herausnehmen. Dies ist hier aber nicht der Fall (siehe Tabelle). 
Tabelle 21: Kommunalitäten

\begin{tabular}{|l|c|c|}
\hline & Anfänglich & Extraktion \\
\hline Frage1 & 1,000 &, 715 \\
Frage2 & 1,000 &, 676 \\
Frage3 & 1,000 &, 783 \\
Frage4 & 1,000 &, 757 \\
Frage5 & 1,000 &, 787 \\
Frage6 & 1,000 &, 619 \\
Frage7 & 1,000 &, 679 \\
Frage8 & 1,000 &, 684 \\
\hline
\end{tabular}

Extraktionsmethode: Hauptkomponentenanalyse

Erklärte Gesamtvarianz

Es werden alle diejenigen Faktoren extrahiert, die ein Eigenwert $>1$ haben.

Tabelle 22: Tabelle 22: Erklärte Gesamtvarianz

\begin{tabular}{|c|c|c|c|c|c|c|c|c|c|}
\hline \multirow{2}{*}{$\begin{array}{l}\text { Kom- } \\
\text { po- } \\
\text { nente }\end{array}$} & \multicolumn{3}{|c|}{ Anfängliche Eigenwerte } & \multicolumn{3}{|c|}{$\begin{array}{l}\text { Summen von qua- } \\
\text { drierten Faktorladun- } \\
\text { gen für Extraktion }\end{array}$} & \multicolumn{3}{|c|}{$\begin{array}{l}\text { Rotierte Summe der } \\
\text { quadrierten Ladungen }\end{array}$} \\
\hline & $\begin{array}{c}\text { Ge- } \\
\text { samt }\end{array}$ & $\begin{array}{c}\% \text { der } \\
\text { Varianz }\end{array}$ & $\begin{array}{l}\text { Kumu- } \\
\text { lierte \% }\end{array}$ & $\begin{array}{l}\text { Ge- } \\
\text { samt }\end{array}$ & $\begin{array}{l}\% \text { der } \\
\text { Vari- } \\
\text { anz }\end{array}$ & $\begin{array}{l}\text { Kumu- } \\
\text { lierte } \\
\% \\
\end{array}$ & $\begin{array}{l}\text { Ge- } \\
\text { samt }\end{array}$ & $\begin{array}{l}\% \text { der } \\
\text { Vari- } \\
\text { anz }\end{array}$ & $\begin{array}{c}\text { Kumu- } \\
\text { lierte } \\
\% \\
\end{array}$ \\
\hline 1 & 2,919 & 36,486 & 36,486 & 2,919 & 36,486 & 36,486 & 2,525 & 31,557 & 31,557 \\
\hline 2 & 1,714 & 21,422 & 57,908 & 1,714 & 21,422 & 57,908 & 2,077 & 25,956 & 57,513 \\
\hline 3 & 1,068 & 13,352 & 71,260 & 1,068 & 13,352 & 71,260 & 1,100 & 13,747 & 71,260 \\
\hline 4 & ,789 & 9,868 & 81,128 & & & & & & \\
\hline 5 &, 537 & 6,717 & 87,845 & & & & & & \\
\hline 6 & ,444 & 5,551 & 93,396 & & & & & & \\
\hline 7 & ,285 & 3,567 & 96,963 & & & & & & \\
\hline 8 & ,243 & 3,037 & 100,000 & & & & & & \\
\hline
\end{tabular}

Extraktionsmethode: Hauptkomponentenanalyse 
Tabelle 23: Komponentenmatrix(a)

\begin{tabular}{|l|c|c|c|}
\hline & \multicolumn{3}{|c|}{ Komponente } \\
\cline { 2 - 4 } & 1 & 2 & 3 \\
\hline Frage1 &, 830 &,- 138 &, 083 \\
Frage2 &,- 296 &, 714 &,- 280 \\
Frage3 &, 734 &, 425 &,- 252 \\
Frage4 &, 185 &,- 002 &, 850 \\
Frage5 &, 783 &, 408 &,- 089 \\
Frage6 &, 492 &,- 604 &,- 113 \\
Frage7 &, 622 &, 459 &, 285 \\
Frage8 &,- 573 &, 512 &, 307 \\
\hline
\end{tabular}

Extraktionsmethode: Hauptkomponentenanalyse.

a 3 Komponenten extrahiert

Tabelle 24: Rotierte Komponentenmatrix(a)

\begin{tabular}{|l|c|c|c|}
\hline & \multicolumn{3}{|c|}{ Komponente } \\
& 1 & 2 & 3 \\
\hline Frage1 &, 598 &,- 565 &, 194 \\
Frage2 &, 182 &, 726 &,- 341 \\
Frage3 &, 864 &,- 081 &,- 171 \\
Frage4 &, 077 &,- 017 &, 867 \\
Frage5 &, 881 &,- 106 &,- 001 \\
Frage6 &, 075 &,- 783 &,- 027 \\
Frage7 &, 745 &, 064 &, 347 \\
Frage8 &,- 211 &, 771 &, 212 \\
\hline
\end{tabular}

Extraktionsmethode: Hauptkomponentenanalyse.

Rotationsmethode: Varimax mit Kaiser-Normalisierung.

a Die Rotation ist in 4 Iterationen konvergiert. 


\section{Faktor 1:}

- Item 1: Das Video berührt mich gefühlsmäßig. (+)

- Item 3: Es ist mir peinlich. (+)

- Item 5: Es stößt mich ab. (+)

- Item 7: Es deprimiert mich. (+)

\section{Faktor 2:}

- Item 1: Das Video berührt mich gefühlsmäßig. (-)

- Item 2: Es ist mir gleichgültig. (+)

- Item 6: Ich finde es hilfreich, die Entgiftung durchzuhalten. (-)

- Item 8: Es hat für mich keine Bedeutung, diese Aussage stimmt. (+)

\section{Faktor 3:}

- Item 4: Es macht mir Suchtdruck

Tabelle 25: Komponententransformationsmatrix

\begin{tabular}{|c|c|c|c|}
\hline Komponente & 1 & 2 & 3 \\
\hline 1 &, 823 &,- 554 &, 129 \\
2 &, 562 &, 827 &,- 036 \\
3 &,- 087 &, 102 &, 991 \\
\hline
\end{tabular}

Extraktionsmethode: Hauptkomponentenanalyse

Rotationsmethode: Varimax mit Kaiser-Normalisierung 
Tabelle 26: Zusammenhang von Traumatisierung und Suchterkrankung (zitiert aus: Lüdecke et al. 2010, S. 14-15)

\begin{tabular}{|c|c|c|c|c|}
\hline Studie & Jahr & Anzahl & Traumatisierung & Suchtmittelkonsum \\
\hline Mullen et al. & 1993 & $\begin{array}{l}492 \\
\text { Frauen }\end{array}$ & $\begin{array}{l}\text { sexueller Missbrauch } \\
\text { in der Kindheit }\end{array}$ & $\begin{array}{l}\text { Alkohol-Missbrauch/- } \\
\text { Abhängigkeit: aktuell } \\
34 \% \text { versus AB 9\% } \\
\text { Drogen-/Medikamenten- } \\
\text { Missbrauch/- } \\
\text { Abhängigkeit: aktuell } \\
25 \% \text { versus AB } 4 \%\end{array}$ \\
\hline Silvermann et al. & 1996 & $\begin{array}{l}375 \\
\text { Männer } \\
\text { und } \\
\text { Frauen }\end{array}$ & $\begin{array}{l}\text { sexueller Missbrauch } \\
\text { und körperliche } \\
\text { Misshandlung }\end{array}$ & 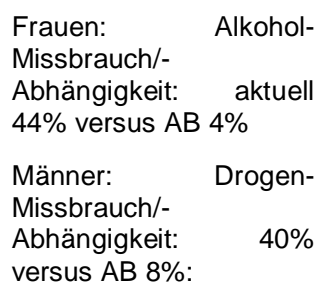 \\
\hline Ducan et al. & 1996 & $\begin{array}{l}4000 \\
\text { Frauen }\end{array}$ & $\begin{array}{l}\text { körperliche } \\
\text { Misshandlung } \\
\text { in der Kindheit }\end{array}$ & 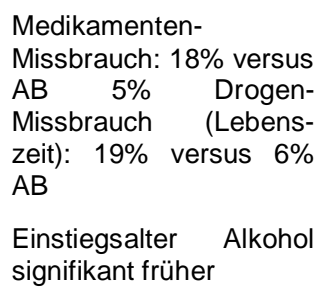 \\
\hline Wilsnack et al. & 1997 & $\begin{array}{l}1099 \\
\text { Frauen }\end{array}$ & $\begin{array}{l}\text { sexueller Missbrauch } \\
\text { in der Kindheit }\end{array}$ & $\begin{array}{l}\text { Alkohol-Abhängigkeit: } \\
\text { aktuell } 19 \% \text { versus } A B \\
6 \% \\
\text { Konsum illegaler Dro- } \\
\text { gen (Lebenszeit): } \\
35 \% \text { versus } A B 14 \%\end{array}$ \\
\hline Clark et al. & 1997 & $\begin{array}{l}183 \\
\text { Jugendliche }\end{array}$ & $\begin{array}{l}6 \text { bis } 12 \text {-fach erhöhte } \\
\text { Wahrscheinlichkeit für } \\
\text { körperliche Misshand- } \\
\text { lung } \\
\text { 18- bis } 21 \text {-fach erhöh- } \\
\text { te Wahrscheinlichkeit } \\
\text { für sexuellen Miss- } \\
\text { brauch }\end{array}$ & $\begin{array}{l}100 \% \text { Alkoholabhängig- } \\
\text { keit }\end{array}$ \\
\hline
\end{tabular}




\section{Tabelle 26 (Fortsetzung): Zusammenhang von Traumatisierung und}

\section{Suchterkrankung (zitiert aus: Lüdecke et al. 2010, S. 14-15)}

\begin{tabular}{|c|c|c|c|c|}
\hline Studie & Jahr & Anzahl & Traumatisierung & Suchtmittelkonsum \\
\hline Schmidt & 2000 & $\begin{array}{l}\text { } 215 \\
\text { opiatabhängige Männer } \\
\text { und Frauen }\end{array}$ & $\begin{array}{l}\text { Bei } 60 \% \text { der Frauen } \\
\text { versus } 11 \% \text { (studie- } \\
\text { rende Kontrollgrup- } \\
\text { pe). Bei } 25 \% \text { der } \\
\text { Männer versus } 6 \% \\
\text { (studierende Kon- } \\
\text { trollgruppe). Opiat- } \\
\text { Abhängige: häufiger } \\
\text { Inzest, schwerer, } \\
\text { häufiger und in jün- } \\
\text { gerem Alter stattge- } \\
\text { habter sexueller } \\
\text { Missbrauch in der } \\
\text { Kindheit }\end{array}$ & Opiat-Abhängigkeit \\
\hline Schäfer et al. & 2000 & $\begin{array}{l}100 \\
\text { Männer } \\
\text { und } \\
\text { Frauen }\end{array}$ & $\begin{array}{l}\text { erzwungener Ge- } \\
\text { schlechtsverkehr in } \\
\text { der Kindheit: } \\
50 \% \text { der Frauen, } \\
40 \% \text { der Männer }\end{array}$ & $\begin{array}{l}\text { polyvalenter Suchtmittel- } \\
\text { Konsum }\end{array}$ \\
\hline Kendler et al. & 2000 & $\begin{array}{l}700 \\
\text { weibliche Zwillingspaare }\end{array}$ & sexueller Missbrauch & $\begin{array}{l}\text { 6- bis 7-fach erhöhte } \\
\text { Wahrscheinlichkeit für } \\
\text { Entwicklung einer späteren } \\
\text { Alkohol- oder Drogen- } \\
\text { Abhängigkeit }\end{array}$ \\
\hline $\begin{array}{l}\text { Simpson } \quad u \text {. } \\
\text { Miller }\end{array}$ & 2002 & $\begin{array}{l}\text { weibliche Patienten in } \\
\text { klinischer Suchtbehand- } \\
\text { lung }\end{array}$ & $\begin{array}{l}\text { kindlicher sexueller } \\
\text { Missbrauch }\end{array}$ & $\begin{array}{l}\text { durchschnittliche Prävalenz } \\
\text { bei } 45 \% \text { der suchtmittelab- } \\
\text { hängigen Frauen in klini- } \\
\text { scher Behandlung }\end{array}$ \\
\hline Krausz u. Briken & 2002 & 75 Frauen & $\begin{array}{l}40 \% \text { sexueller Miss- } \\
\text { brauch } \\
72 \% \text { körperliche } \\
\text { Misshandlung } \\
80 \% \text { emotionale } \\
\text { Misshandlung }\end{array}$ & $\begin{array}{l}\text { manifeste Opiatabhängig- } \\
\text { keit }\end{array}$ \\
\hline $\begin{array}{l}\text { Simpson } \\
\text { Miller }\end{array}$ & 2002 & 53 Studien & $\begin{array}{l}27 \% \text { bis } 67 \% \text { der } \\
\text { Frauen sexuell miss- } \\
\text { braucht, } 33 \% \text { der } \\
\text { Frauen körperlich } \\
\text { missbraucht } \\
95 \text { bis } 13 \% \text { der Män- } \\
\text { ner sexuell miss- } \\
\text { braucht, } 53 \% \text { körper- } \\
\text { lich missbraucht }\end{array}$ & $\begin{array}{l}\text { Substanzmittelabhängigkeit } \\
\text { (nicht näher definiert) }\end{array}$ \\
\hline Kemner et al. & 2004 & $\begin{array}{l}313 \text { Männer und Frauen, } \\
\text { die sich in einer Entwöh- } \\
\text { nungsbehandlung befan- } \\
\text { den }\end{array}$ & $\begin{array}{l}\text { Körperliche } \\
\text { handlung in der } \\
\text { Kindheit }\end{array}$ & $\begin{array}{l}14 \% \text { der } \quad \text { Alkohol- } \\
\text { Abhängigen versus } 5 \% \mathrm{AB}\end{array}$ \\
\hline
\end{tabular}


Tabelle 27: Zusammenhang von Traumatisierung, Posttraumatischer

Belastungsstörung und Substanzmittel-Konsum (zitiert aus: Lüdecke et al. 2010, S. 17-18)

\begin{tabular}{|c|c|c|c|c|c|}
\hline Studie & Jahr & Anzahl & Traumatisierung & PTBS & Suchtmittelkonsum \\
\hline $\begin{array}{l}\text { NCS- Studie } \\
\text { Kessler et al. }\end{array}$ & 1995 & $\begin{array}{l}5877 \\
\text { Männer und Frau- } \\
\text { en zwischen } 15 \\
\text { und } 54 \text { Jahren }\end{array}$ & $\begin{array}{l}\text { 60\% der Männer } \\
50 \% \text { der Frauen }\end{array}$ & $\begin{array}{l}\text { Männer: } \\
5 \% \text { Lebenszeit } \\
\text { Frauen: } \\
10 \% \text { Lebenszeit } \\
\text { Höchstrisiko } \\
\text { Vergewaltigung: } \\
65 \% \text { Männer, } \\
45 \% \text { Frauen }\end{array}$ & $\begin{array}{l}\text { 2,5-fach erhöhte Wahr- } \\
\text { scheinlichkeit Alkohol } \\
\text { (Frauen) } \\
\text { erhöhte Wahrschein- } \\
\text { lichkeit Drogen (Frau- } \\
\text { en) 2-fach erhöhte } \\
\text { Wahrscheinlichkeit } \\
\text { Alkohol (Männer) } \\
\text { 3-fach erhöhte Wahr- } \\
\text { scheinlichkeit Drogen } \\
\text { (Männer) }\end{array}$ \\
\hline $\begin{array}{l}\text { Early Adult- } \\
\text { hood Research } \\
\text { Project } \\
\text { Giaconia et al. }\end{array}$ & 1995 & $\begin{array}{l}384 \\
\text { 18-jährige Männer } \\
\text { und Frauen }\end{array}$ & $\begin{array}{l}43 \% \\
\text { jeweils } \quad \begin{array}{l}\text { Männer } \\
\text { und }\end{array} \quad \text { Frauen }\end{array}$ & $\begin{array}{l}\text { Frauen: } \\
10,5 \% \\
\text { Männer: } \\
2,1 \%\end{array}$ & $\begin{array}{l}\text { 4,5-fach erhöhte Wahr- } \\
\text { scheinlichkeit, eine } \\
\text { Alkohol-Abhängigkeit } \\
\text { zu entwickeln } \\
\text { 8,8-fach erhöhte Wahr- } \\
\text { scheinlichkeit, eine } \\
\text { Drogen-Abhängigkeit } \\
\text { zu entwickeln }\end{array}$ \\
\hline $\begin{array}{l}\text { ECA-Survey } \\
\text { Helzer et al. }\end{array}$ & 1987 & $\begin{array}{l}3000 \\
\text { Männer und } \\
\text { Frauen }\end{array}$ & / & $\begin{array}{l}\text { Männer: } \\
0,5 \% \quad \text { Lebens- } \\
\text { zeit } \quad \text { Frauen: } \\
1,3 \% \\
\text { Lebenszeit }\end{array}$ & $\begin{array}{l}\text { Bei PTBS: } \\
\text { 1,5-fach erhöhte Wahr- } \\
\text { scheinlichkeit, eine } \\
\text { Drogenerkrankung zu } \\
\text { entwickeln (bei Frauen) } \\
\text { 3-fach erhöhte Wahr- } \\
\text { scheinlichkeit, eine } \\
\text { Alkohol-Abhängigkeit } \\
\text { zu entwickeln (bei } \\
\text { Frauen) } \\
\text { fach erhöhte Wahr- } \\
\text { scheinlichkeit, eine } \\
\text { Drogenerkrankung zu } \\
\text { entwickeln (bei Män- } \\
\text { nern) } 2- \\
\text { fach erhöhte Wahr- } \\
\text { scheinlichkeit, eine } \\
\text { Alkoholerkrankung zu } \\
\text { entwickeln (bei Män- } \\
\text { nern) }\end{array}$ \\
\hline Breslau et al. & 1998 & $\begin{array}{l}1000 \text { Männer } \\
\text { und Frauen }(21 \\
\text { bis 30J.) Nachun- } \\
\text { tersuchung nach } \\
\text { 5J. an } 55 \text { Männern } \\
\text { und Frauen }\end{array}$ & $\begin{array}{l}\text { innerhalb des Untersu- } \\
\text { chungszeitraumes } \\
25 \% \text { Traumatisierungen } \\
\text { davon } \quad \rightarrow \mathbf{1 0 \%} \\
\rightarrow \mathbf{1 5 \%}\end{array}$ & $7 \%$ & $\begin{array}{l}\text { Bei PTBS: Im Untersu- } \\
\text { chungszeitraum } \\
\text { fach erhöhtes Risiko, } \\
\text { eine Sucht zu entwi- } \\
\text { ckeln }(8,6 \% \text { mit PTBS } \\
\text { versus } 2,2 \% \text { ohne } \\
\text { PTBS) } \\
\text { bei } \rightarrow 10 \% \text { keine Abhängig- } \\
\text { keitserkankung, bei } \rightarrow 15 \% \text { im } \\
\text { Vorfeld Abhängigkeits- } \\
\text { erkankung bekannt }\end{array}$ \\
\hline
\end{tabular}


Tabelle 27 (Fortsetzung): Zusammenhang von Traumatisierung, Posttraumatischer Belastungsstörung und Substanzmittel-Konsum (zitiert aus: Lüdecke et al. 2010, S. 17-18)

\begin{tabular}{|c|c|c|c|c|c|}
\hline Studie & Jahr & Anzahl & Traumatisierung & PTBS & Suchtmittelkonsum \\
\hline North et al. & 1999 & $\begin{array}{l}182 \\
\text { Männer und } \\
\text { Frauen }\end{array}$ & $\begin{array}{l}100 \% \text { Bombenan- } \\
\text { schlag auf Regie- } \\
\text { rungsgebäude in } \\
\text { Oklahoma City }\end{array}$ & $\begin{array}{l}62 \text { Personen } \\
\text { ca. } 30 \%\end{array}$ & $\begin{array}{l}\text { davon Drogen in der } \\
\text { Vorgeschichte: } 41 \% \\
\text { davon Alkohol in der } \\
\text { Vorgeschichte: } 37,5 \% \\
\text { keine psychiatrische } \\
\text { Diagnose: } 27 \%\end{array}$ \\
\hline Kutscher et al. & 2002 & $\begin{array}{l}\text { Männer und } \\
\text { Frauen }\end{array}$ & / & $\begin{array}{l}\text { 8\% Männer } \\
22 \% \text { Frauen }\end{array}$ & Alkohol-Abhängigkeit \\
\hline Zumbeck et al. & 2003 & $\begin{array}{l}\text { Hamburger } \\
\text { Prostituierte }\end{array}$ & $\begin{array}{lr}83 \% & \text { schwere } \\
\text { Traumat in } \quad \text { der } \\
\text { Prostitution } \quad(61 \% \\
\text { sexueller Miss- } \\
\text { brauch, 60\% körper- } \\
\text { liche Misshandlung) }\end{array}$ & $\begin{array}{l}59 \% \text { davon: } \\
65 \% \text { körperliche } \\
\text { Misshandlung } \\
\text { davon: } 48 \% \\
\text { sexueller Miss- } \\
\text { brauch in der } \\
\text { Kindheit }\end{array}$ & $\begin{array}{l}74 \% \text { Substanzmittel- } \\
\text { Abhängigkeit } \\
\text { (nicht näher definiert) } \\
\text { (Lebenszeit) }\end{array}$ \\
\hline Mills et al. & 2005 & 495 & / & $42 \%$ & $\begin{array}{l}\text { Opiat-Abhängigkeit } \\
100 \%\end{array}$ \\
\hline $\begin{array}{l}\text { Traumab- } \\
\text { Studie } \\
\text { Driessen et al. }\end{array}$ & 2008 & 459 & $67 \%$ & $\begin{array}{l}\text { insgesamt } \\
25,3 \% \\
\text { davon Alkohol: } \\
15 \% \\
\text { davon Drogen } \\
30 \% \\
\text { davon Alkohol u. } \\
\text { Drogen } \\
34 \%\end{array}$ & $\begin{array}{l}\text { Suchtmittel: } \\
100 \% \text { Beginn des } \\
\text { Alkohol-Konsums. } \\
4 \text { bis } 7 \text { Jahre eher }\end{array}$ \\
\hline
\end{tabular}


Anhang B

Patienteneinverständniserklärungen und Patientenaufklärungen

Dorothee Pletke, Ärztin am NLKH Göttingen, Rosdorfer Weg 70, 37081 Göttingen

\title{
6.2.1 Patienteneinverständniserklärung zur Studie
}

\author{
„Videodokumentation und -konfrontation - ein wirksames Mittel zur Verminderung \\ der Abbruchquote beim stationären Drogenentzug“
}

Ich, wurde von meinem Arzt vollständig über Wesen, Bedeutung und Tragweite der medizinischen Untersuchung mit o.g. Titel aufgeklärt. Ich habe den Aufklärungstext gelesen und verstanden. Ich hatte die Möglichkeit, Fragen zu stellen und habe die Antworten verstanden und akzeptiere sie. Meine Ärztin hat mich über die mit der Teilnahme an der Untersuchung verbundenen Risiken und die möglichen Nutzen informiert.

Ich hatte ausreichend Zeit, mich zur Teilnahme an dieser Studie zu entscheiden und weiß, dass die Teilnahme daran freiwillig ist. Ich weiß, dass ich jederzeit ohne Angabe von Gründen diese Zustimmung widerrufen kann, ohne dass sich dieser Entschluss nachteilig auf die spätere Behandlung durch meine Ärztin auswirken wird. Mir ist bekannt, dass meine persönlichen Daten in verschlüsselter Form gespeichert werden. Im Falle meines Widerrufs werden Fragebögen, Daten und Videoaufzeichnung gelöscht. Ich habe eine Kopie der Patienteninformation und dieser Einwilligungserklärung erhalten. Ich erkläre hiermit meine freiwillige Teilnahme an dieser klinischen Studie.

Unterschrift Patient/Patientin Unterschrift Aufklärender Arzt/Ärztin

Ort, Datum

Ort,

Datum 


\title{
6.2.2 Patientenaufklärung zur Studie
}

\author{
„Videodokumentation und -konfrontation - ein wirksames Mittel zur Verminderung \\ der Abbruchquote beim stationären Drogenentzug?"
}

Liebe Patientin, lieber Patient,

wir möchten Sie über eine Studie aufklären, die wir hier auf der Drogenentzugsstation durchführen, um die Therapieabbrüche zu vermindern. Die Behandlung von Suchterkrankungen ist schwierig und nicht selten kommt es zum Behandlungsabbruch. In der wissenschaftlichen Literatur liegen die Abbruchquoten auf Drogenentzugsstationen bei $50 \%$ und höher. Es gibt zahlreiche Untersuchungen, womit diese hohe Abbruchquote zusammenhängen könnte und wie man das Durchhaltevermögen der Patienten verbessern könnte, ohne dass es hier bisher Fortschritte gegeben hätte. Einzig der Narkoseentzug („Turboentzug") ist günstiger, wird aber erfahrungsgemäß von den Krankenkassen nicht bezahlt. Es ist daher wichtig, Möglichkeiten zu finden, um das Durchhaltevermögen unserer Patienten zu verbessern. Aus diesem Grund möchten wir gucken, ob die Patienten bei uns die Behandlung besser durchhalten, wenn wir sie bei Aufnahme per Video filmen und innen ihren Film am sogenannten Nulltag, das heißt dem Tag an dem sie von der zu entgiftenden Substanz nichts mehr bekommen, zeigen. Von einigen Patienten wird also bei der Aufnahme ein Video aufgenommen, bei anderen Patienten ist das nicht der Fall. In beiden Patientengruppen (mit Video/ohne Video) soll dann die Abbruchquote verglichen werden. Die Zuteilung der Patienten in die beiden Gruppen erfolgt nach dem Zufallsprinzip. Wollen diejenigen, die per Video aufgenommen wurden, ihre Behandlung schon vor dem „Nulltag“ abbrechen, so sollten sie ihr Video dann anschauen, um den Entschluss, die Entgiftung zu beenden gegebenenfalls zu ändern. Im Anschluss an das gemeinsame Anschauen des Videos mit der Stationsärztin erhalten sie einen Fragebogen zur Beurteilung der Videokonfrontation. Außerdem werden Sie mit der Ärztin in einem oder auch mehreren Gesprächen Gelegenheit haben über den Einfluss der Videoaufnahme auf sie zu sprechen. Alle anderen üblichen Hilfen, um die Suchttherapie durchzuhalten, kommen Ihnen natürlich auch zu Gute wie z. B. Akupunktur - wenn gewünscht - oder der Einsatz von Medikamenten wie Aponal, Clonidin oder Dominal. Sie nehmen freiwillig an der Studie teil und können ihr Einverständnis zur Teilnahme jederzeit widerrufen. Dann werden alle ihre Daten gelöscht. Dies oder Nichtteilnahme ohne Angabe von Gründen hat keine Nachteile für die weitere Behandlung. Alle Daten und Inhalte der Untersuchung unterliegen der ärztlichen Schweigepflicht. Mit den Daten wird vertraulich umgegangen und zur Auswertung werden diese pseudonymisiert. Gelöschte oder pseudonymisierte Daten stehen später nicht mehr zur Verfügung. Der Datenschutz wird eingehalten. In Notfällen sind eure Stationsärztin und die Prüfärztin sowie die Oberärztin der Station jederzeit ansprechbar.

Dorothee Pletke, Prüfärztin

Christel Lüdecke, Oberärztin 
Dorothee Pletke, Ärztin am NLKH Göttingen, Rosdorfer Weg 70, 37081 Göttingen

\subsubsection{Patientenaufklärung zur Studie (Kontrollgruppe)}

\section{„Videodokumentation und -konfrontation - ein adäquates Mittel zur Verminderung der Abbruchquote beim stationären Drogenentzug“}

Liebe Patientin, lieber Patient,

wir möchten Sie über eine Untersuchung aufklären, die wir hier auf der Drogenentzugsstation durchführen, um die Therapieabbrüche zu vermindern. Die Behandlung von Suchterkrankungen ist schwierig und nicht selten kommt es zum Behandlungsabbruch. In der wissenschaftlichen Literatur liegen die Abbruchquoten auf Drogenentzugsstationen ungefähr bei $50 \%$. Es gibt zahlreiche Untersuchungen dazu, womit diese hohe Abbruchquote zusammenhängen könnte und wie man das Durchhaltevermögen der Patienten verbessern könnte, ohne dass es hier bisher wesentliche Fortschritte gegeben hätte. Es ist daher wichtig, Möglichkeiten zu finden, um das Durchhaltevermögen unserer Patienten zu verbessern. Aus diesem Grund möchten wir gucken, ob die Patienten die Behandlung besser durchhalten, wenn sie bei Aufnahme per Video gefilmt werden und ihren Film am sogenannten Nulltag anschauen können. Nulltag heißt der Tag, an dem der jeweilige Patient von der zu entgiftenden Substanz nichts mehr bekommt. Von einigen Patienten wird also bei der Aufnahme eine Videoaufnahme gemacht, bei anderen Patienten ist das nicht der Fall. Die Zuteilung der Patienten in die beiden Gruppen erfolgt nach dem Zufallsprinzip. In beiden Patientengruppen (mit Video/ohne Video) solle dann die Abbruchquote verglichen werden. Wollen diejenigen, die per Video aufgenommen wurden, ihre Behandlung schon vor dem "Nulltag" abbrechen, so sollten sie ihr Video dann anschauen, um den Entschluss, die Entgiftung zu beenden gegebenenfalls zu ändern.

Sie nehmen auch im Sinne einer Vergleichsgruppe freiwillig an der Studie teil und können Ihr Einverständnis zur Teilnahme jederzeit widerrufen. Dies oder Nichtteilnahme ohne Angabe von Gründen hat keine Nachteile für die weitere Behandlung. Alle Daten und Inhalte der Untersuchung unterliegen der ärztlichen Schweigepflicht. Mit den Daten wird vertraulich umgegangen und zur Auswertung werden diese anonymisiert. Die Auflagen des Datenschutzes werden eingehalten. In Notfällen sind ihre Stationsärztin und die Prüfärztin sowie die Oberärztin der Station jederzeit ansprechbar.

Dorothee Pletke, Prüfärztin;

Christel Lüdecke, Oberärztin 
Dorothee Pletke, Ärztin am NLKH Göttingen, Rosdorfer Weg 70, 37081 Göttingen

6.2.4 Patienteneinverständniserklärung zur Studie (Kontrollgruppe)

\begin{abstract}
„Videodokumentation und -konfrontation - ein adäquates Mittel zur Verminderung der Abbruchquote beim stationären Drogenentzug“"
\end{abstract}

Ich,

wurde von meinem Arzt vollständig über Wesen, Bedeutung und Tragweite der medizinischen Untersuchung mit o.g. Titel aufgeklärt. Ich habe den Aufklärungstext gelesen und verstanden. Ich hatte die Möglichkeit, Fragen zu stellen und habe die Antworten verstanden und akzeptiere sie. Meine Ärztin hat mich über den mit der Teilnahme an der Untersuchung verbundenen möglichen Nutzen informiert. Meine Daten werden in der Untersuchung als Kontrolldaten verwandt.

Ich hatte ausreichend Zeit, mich zur Teilnahme an dieser Untersuchung zu entscheiden und weiß, dass die Teilnahme daran freiwillig ist. Ich weiß, dass ich jederzeit ohne Angabe von Gründen diese Zustimmung widerrufen kann, ohne dass sich dieser Entschluss nachteilig auf die spätere Behandlung durch meine Ärztin auswirken wird. Mir ist bekannt, dass meine persönlichen Daten in verschlüsselter Form gespeichert werden. Ich habe eine Kopie der Patienteninformation und dieser Einwilligungserklärung erhalten. Ich erkläre hiermit meine freiwillige Teilnahme an dieser klinischen Studie.

Unterschrift Patient/Patientin

Unterschrift Aufklärender Arzt/Ärztin

Ort, Datum.

Ort, Datum 
6.3

\section{Anhang C}

\section{Erhebungsbögen}

Dorothee Pletke, Ärztin am NLKH Göttingen, Rosdorfer Weg 70, 37081 Göttingen

Datum:

Uhrzeit:

Station:

Patient Code:

geb.:

Geschlecht:

$\begin{array}{ll}\square & m \\ \square & w\end{array}$

Wartezeit auf den Entgiftungsplatz

$$
\begin{aligned}
& \text { ㅁ } 0 \text { = ohne } \\
& \text { ㅁ } \quad 1=1-2 \text { Wochen } \\
& \text { ㄴ } 2=2-3 \text { Wochen } \\
& \text { ㅁ } 3=3-4 \text { Wochen } \\
& \text { ㅁ } 4=>4 \text { Wochen }
\end{aligned}
$$

Eingewiesen durch:

Diagnose:

Begleitdiagnosen:

somatisch + psychiatrisch

Aufnahmeanlass

Therapiemotivation, Ziele

\begin{tabular}{|l|}
\hline \\
\hline 2 \\
\hline 2 \\
\hline
\end{tabular}




\begin{tabular}{|l|l|l|l|l|l|l|}
\hline Suchtanamnese & & & & & & \\
\hline & $\begin{array}{l}\text { Suchtstoff } \\
\text { seit }\end{array}$ & regelm. & Unregelm & Menge & Xmal/Tag & zuletzt \\
\hline Heroin: & & & & & & \\
\hline Kokain: & & & & & & \\
\hline Benzod. & & & & & & \\
\hline Cannabis: & & & & & & \\
\hline Alkohol: & & & & & & \\
\hline Amphetamine: & & & & & & \\
\hline Metha./Pola: & & & & & & \\
\hline Nikotin: & & & & & & \\
\hline Sonst: & & & & & & \\
\hline
\end{tabular}

Sozialanamnese (Heim?, Schule?, Berufsausbildung?, Arbeitsstelle?, Wohnsituation), Bezugspersonen?

Soz.Sit.: ledig, verheiratet, geschieden, getrennt lebend. Mietwhg,; Eigentum; obdachlos, lebt - allein, mit Partner, mit Eltern, Kind(ern), mit....

lebt von - Sozialhilfe, Arbeitslosengeld/-hilfe, Einkommen, Rente ( Euro)

Berufstätig als

Biographie: Eltern:

Geschwister:

Heimkind/Pflegefam.:

Fam- Gewalt:

Traumata:

Schule,- Abschluss

Beruf:

Auffälligkeiten 
Haftstrafen (von - bis, wo? Grund?)

$\S 35 ? \mathrm{~J} / \mathrm{Nn} \quad$ Bewährung: Nn/J Dauer:

Offene Verfahren?

Familienanamnese: Sucht?, Psych. Erkr.? Somat. Erkr.?

Interessen

\begin{tabular}{|ll|}
\hline Internistische Anamnese: & $\square$ Hepatitis A \\
& $\square$ Hepatitis B \\
& $\square$ Hepatitis C \\
& $\square$ HIV \\
\hline
\end{tabular}

\section{Psychiatrische Vorgeschichte:}

Suizidversuch in der Vorgeschichte?

Regelmäßiger Drogenkonsum in den letzten Wochen (Stoffgruppen, jeweilige Tagesdosis?)

Letzte Drogeneinnahme (wann?, welche?) 


\begin{tabular}{|l|l|l|l|}
\hline Frühere Entgiftung /drogenfreie Zeit & & drogenfreie Zeit? \\
\hline Von-bis & Wo & Erfolg? & von-bis \\
\hline & & & \\
\hline & & & \\
\hline & & & \\
\hline
\end{tabular}

Medikamentöse Therapie

( $\mathrm{P}=$ Polamidon, $\mathrm{M}=$ Methadon, $\mathrm{C}=$ Codein, $\mathrm{B}=$ Benzodiazepine, $\mathrm{N}=$ Naltrexon)

\begin{tabular}{|l|l|l|}
\hline von-bis & Art & Wo \\
\hline & & \\
\hline & & \\
\hline & & \\
\hline & & \\
\hline & & \\
\hline
\end{tabular}

Langzeittherapien

\begin{tabular}{|l|l|l|}
\hline von-bis & wo & Ergebnis \\
\hline & & \\
\hline & & \\
\hline & & \\
\hline & & \\
\hline & & \\
\hline
\end{tabular}

Psychischer Befund: 
Körperlicher Untersuchungsbefund

Größe: $\quad$ Gewicht: AZ:

RR: Puls: Zahnstatus: 
Abbass A (2004): Small-group videotape training for psychotherapy skills development. Acad Psychiatry $\underline{28}(2), 151-155$

Alger I (1969): Therapeutic use of videotape playback. J Nerv Ment Dis $148(4)$, 430-436

Alger I, Hogan P (1967): The impact of videotape recording on involvement in group therapy. J Psychoanal Groups $\underline{2}, 50-56$

Alger I, Hogan P (1969): Enduring effects of videotape playback experience on family and marital relationships. Am J Orthopsychiatry $\underline{39}$ (1), 86-98

Angermeyer M C, Schulze B, Dietrichs S (2003): Courtesy stigma - a focus group study of relatives of schizophrenia patients. Soc Psychiatry Psychiatr Epidemiol $\underline{38}$ (10), 593-602

Armenian SH, Chutuape MA, Stitzer ML (1999): predictors of discharges against medical advice from a short-term hospital detoxification unit. Drug Alcohol Depend $\underline{56}(1), 1-8$

Aronson E, Wilson TD, Akert RM : Social Psychology. Prentice Hall, Upper Saddle River, NJ 2002

Backmund M, Meyer K, Eichenlaub D, Schütz CG (2001): Predictors for completing an inpatient detoxification program among intravenous heroin users, methadone substituted and codeine substituted patients. Drug Alcohol Depend $\underline{64}$ (2), 173-180

Backmund M, Schütz CG, Meyer K, Eichenlaub D, Soyka M (2003): Alcohol consumption in Heroin Users, Methadone-Substituted and Codeine-Substituted $\mathrm{Pa}$ tients- Frequency and Correlates of Use. Eur Addict Res $\underline{9}$ (1), 45-50

Backmund M, Meyer K, Henkel C, Soyka M, Reimer J, Schütz CG (2005): COConsumption of Benzodiazepines in Heroin Users, Methadone Substituted, and Codeine-Substituted Patients. J Addict Dis 24 (4), 17-29

Backmund M, Bernhard-Wehmeier W, Meyer K, Abeck C, Holzke D und das VillaTeam 1991-2006 (2006): 15 Jahre qualifizierte Drogenentzugsbehandlung an einem Münchner Akutkrankenhaus. Suchtmed Forsch Prax $\underline{8}(1), 23-28$

Bailey K G (1970): Audiotape self confrontation in group psychotherapy. Psychol Rep $\underline{27}$ (2), 439-444

Bailey K G, Sowder W T (1970): Audiotape and videotape self-confrontation in Psychotherapy. Psychol Bull $\underline{74}$ (2), 127-137 
Baker T B, Udin H, Vogler R E (1975): The effects of videotaped modelling and self-confrontation on the drinking behaviour of alcoholics. Int J Addict 10 (5), 779793

Banbery J, Wolff K, Raistrick D (2000): Dihydrocodein: a Useful tool in the detoxification of methadone maintained patients. J Subst Abuse Treat 19 (3), 301-305

Barth J, Bengel $\mathrm{J}$ : Prävention durch Angst? Bundeszentrale für gesundheitliche Aufklärung (BZgA), Köln 1998

Bartter T, Gooberman LL (1996): Rapid opiate detoxification. Am J Drug Alcohol Abuse $\underline{22}$ (4), 489-495

Basdekis-Josza R, Krausz M (2002): Schizophrenie und Opiatabhängigkeit. Psychoneuro $\underline{28}, 367-370$

Beebe B (2005): Mother-infant research informs mother-infant treatment. Psychoanal Study Child $\underline{60}, 7-46$

Behrendt K, Bonorden-Kleij K, Krausz M, Degkwitz P, Kellermann B (1993): Niederschwelliger Drogenentzug: Konzept, Erfahrungen, Konsequenzen. Dtsch Arztebl $\underline{90}, 147-151$

Berger M M: Videotape technique in psychiatric training and treatment. Bruner/Mazel, New York 1970

Berger M M, Sherman B, Spalding J, Westlake R (1968): The use of videotape with psychotherapy groups in a community mental health service program. Int $\mathrm{J}$ Group Psychother 18 (4), 504-515

Berman AH, Forsberg L, Durbeej N, Källmén H, Hermansson U (2010): Singlesession motivational interviewing for drug detoxification inpatients: effects on selfefficacy, stage of change and substance use. Subst Use Misuse 45 (3), 384-402

Bertschy G (1995): Methadone maintenacnce treatment: an update. Eur Arch Psychiatry Clin Neurosci 245 (2), 114-124

Bickel W, Stitzer M, Bigelow G, Liesbon IA, Jasinski DR, Johnson RE (1988): A clinical trial of Buprenorphin: Comparison with Methadone in the Detoxifikation of Heroin addicts. Clin Pharmacol Ther $\underline{43}$ (1), $72-78$

Bilodeau E A , Bilodeau IMcD: Principles of skill acquisition. Academic Press, London 1969

Bless H: Stimmung und Denken: Ein Modell zum Einfluss von Stimmungen auf Denkprozesse. Hans Huber, Bern 1997 
Boehle C, Kindgen-Milles D, Burtscheidt W, Tarnow J, Gaebel W (2000): Antagonisteninduzierte Opiatentgiftung. Nervenarzt $\underline{71}$ (9), 745-759

Bonorden-Kleij K, Behrendt K, Degkwitz P, Krausz M (1993a): Vom Abstinenzparadigma zur praktischen Gesundheitsvorsorge - die Bedeutung des niederschwelligen Entzuges im Drogenhilfesystem. Teil I Krankenhauspsychiatrie 4 (3), 128130

Bonorden-Kleij K, Behrendt K, Degkwitz P, Krausz M (1993b): Vom Abstinenzparadigma zur praktischen Gesundheitsvorsorge - die Bedeutung des niederschwelligen Entzuges im Drogenhilfesystem. Teil II Krankenhauspsychiatrie 4 (4), 175179

Boothby LA, Doering PL (2007): Buprenorphine for Treatment of opioid dependence. Am J Health Syst Pharm $\underline{64}$ (1), 266-272

Borges G, Walters EE, Kessler RC (2000): Associations of substance use, abuse, and dependence with subsequent suicidal behaviour. Am J Epidemiol 151 (8), 781-789

Bortz J: Statistik: Für Human- und Sozialwissenschaftler. 5. Auflage; SpringerVerlag, Berlin 1999

Boyd H S, Sisney V V (1967): Immediate sef- image confrontation and changes in self-concept. J Consult Psychol 31 (3), 291-294

Brack J (2002): Der Einsatz von Buprenorphin in der stationären Entzugsbehandlung. Suchttherapie $\underline{3}, 205-210$

Brack J, Behrendt K (2004): Erfahrungen mit dem Einsatz von Buprenorphin (Subutex) in der qualifizierten stationären Entzugsbehandlung Opiatabhängiger. Suchtmed Forsch Prax $\underline{6}$ (3), 241-248

Brehm JW: A therory of psychological reactance. Academic press, New York 1966

Bremner JD, Scott TM, Delaney RC, Southwick SM, Mason JW, Johnson DR, Innis RB, Mc Carthy G, Charney DS (1993 a): Deficits in short-term memory in posttraumatic stress disorder. Am J Psychiatry 150 (7), 1015-1019

Bremner JD, Southwick SM, Johnson DR, Yehuda R, Charney DS (1993 b): Childhood physical abuse and combat-related posttraumatic stress disorder in Vietnam veterans. Am J Psychiatry 150 (2), 235-239

Breslau N, Kessler RC, Chiloat HD, Schultz LR, Davis GC, Andreski P (1998): Trauma and posttraumatic stress disorder in the community: The 1996 Detroit Area Survey of trauma. Arch Gen Psychiatry 55, 726-732 
Brewer C (1996): Anaesthesia or sedation for drug misusers. Lancet $\underline{347}$ (9005), 904

Brewer C (1997): Ultra-rapid, Antagonist-precipitated opiate detoxification under General anaesthesia or Sedation. Addict Biol 2, 291-302

Brewington V, Smith M, Lipton D (1994): Acupuncture as a detoxification treatment: An analysis oft controlled research. J Subst Abuse Treat 11 (4), 289-307

Bühringer G, Adelsberger F, Heinemann A, Kirschner J, Knauß I, Kraus L, Püschel K, Simon R (1997): Schätzverfahren und Schätzungen zum Umfang der Drogenproblematik in Deutschland. Sucht $\underline{43}$, Sonderheft 79-141

Burnam MA, Stein JA, Golding JM, Siegel JM, Sorensen SB, Forsythe AB, Telles CA (1988): Sexual assault and mental disorders in a community population. $J$ Consult Clin Psychol $\underline{56}$ (6), 843-850

Callaghan RC, Cull R, Vettese LC, Taylor L (2006) : A gendered analysis of Canadian Aboriginal individuals admitted to inpatient substance abuse detoxification : a three-year medical chart review. Am J Addict 15 (5), 380-386

Caplan G: Principles of preventive psychiatry. N Y Basic Books, New York 1964

Carroll KM (2005): Recent advances in the psychotherapy of addictive disorders. Curr Psychiatry Rep $\underline{7}$ (5), 329-336

Chaiken S, Liberman A, Eagly A H: Heuristic and systemic information processing within and beyond the persuasion context; in: Unintended thought: Limits of awareness, intention, and control; hrsg. v. Uleman JS, Bargh JA: Guilford, New York 1989, 242-252

Chiang SC, Chan HY, Chang YY, Sun HJ, Chen WJ, Chen CK (2007): Psychiatric comorbidity and gender difference among treatment-seeking heroin abusers in Taiwan. Psychiatry Clin Neurosci 61 (1), 105-111

Chung K M, Reavis S, Mosconi M, Drewry J, Matthews T, Tasse M J (2006): peermediated social skills training program for young children with high-functioning autism. Res Dev Disabil 28 (4), 423-436

Clark DB, Lesnick L, Hegedus AM (1997): Traumas and other adverse life events in adolescents with alcohol abuse and dependence. J Am Acad Child Aolesc Psychiatry $\underline{36}, 1744-1751$

Clerici M, Carta I (1996): Personality disorders among psychoactive substance users: diagnostic and psychodynamic issues. Eur Addict Res 2 (2), 147-155 
Clore G, Schwarz N, Conway M (1994) The Cognitive Causes and Consequences of Emotion; in: Handbook of Social Cognition, vol.1; hrsg. v. Wyer und TK Srull; Erlbaum, Hillsdale , NJ 1994, 331-417

Comfort M, Sockloff A, Loverro J, Kaltenbach K (2003): Multiple predictors of substance-abusing women's treatment and life outcomes: A prospective longitudinal study. Addict Behav 르, 199-224

Cook CC (1989): Inpatient heroin detoxification: a comment. Br J Addict $\underline{84}$ (11), 1369-1371

Cornelison F, Tausig T (1964): A Study of Self-image experience using videotapes at Delware State Hospital. Del Med J 36, 229-231

Cottler LB, Campbell W, Krishna VAS, Cunningham-Williams RM, Abdallah AB (2005): Predictors of High Rates of Suicidal Ideation Among Drug Users. J Nerv Ment Dis $\underline{193}$ (7), 431-437

Craig RJ, Rogalski C, Veltri D (1982): Predicting treatment dropouts from a drug abuse rehabilitation program. Int J Addict 17 (4), 641-653

Danet B N (1968): Self confrontation in psychotherapy reviewed. Am J Psychother $\underline{22}(2), 245-258$

Darke S, Ross J (2002): Suicide among heroin users: rates, risk factors and methods. Addiction 97, 1383-1394

Darke S, Swift W, Hall W, Ross M (1993): Drug use, HIV risk taking and psychosocial correlates of benzodiazepine use among methadone maintenance clients. Drug Alcohol Depend 34, 67-70

De Lisi LE, Maurizio A, Yost M, Papparozzi C, Fulchino C, Katz CL, Altesman J, Biel M, Lee J, Stevens P (2003): A survey of New Yorkers after the September 11 2001 terrorist attacks. Am J Psychiatry 160, 780-783

De los Cobos JP, Trujols J, Ribalta E, Casas M (1997): Cocaine use immediately prior to entry in an inpatient heroin detoxification unit as a predictor of discharges against medical advice. Am J Drug Alcohol Abuse 23, 267-279

Demmel R (2001): Motivational Interviewing: A Review. Sucht 47 (3), 171-188

Deutsche Gesellschaft für Suchtforschung und Therapie e.V. (Hrsg) (2001): Dokumentationsstandards III für die Evaluation der Behandlung von Abhängigen. Sucht 47. Jahrgang, Sonderheft $\underline{2}, 1-95$

Diekstra RF, Gulbinat W (1993): The epidemiology of suicidal behaviour: a review of three continents. World Health Stat Q $\underline{46}$ (1), 52-68 
Dilling H, Mombour W, Schmidt MH, Schulte-Markwort E: Weltgesundheitsorganisation Internationale Klassifikation psychischer Störungen ICD-10, Kapitel V (F); Diagnostische Kriterien für Forschung und Praxis. 2. Auflage; Hans Huber Verlag, Bern 2000

Dixon L, McNary S, Lehman A (1997): One-Year follow-up of secondary versus primary mental disorder in persons with comorbid substance use disorders. Am J Psychiatry $\underline{154}(11), 1610-2$

Dobler-Mikola A, Pfeifer S, Müller V Uchtenhagen A (2000): Vergleich Methadonund Heroinverschreibung in der Schweiz. Suchttherapie 1, 63-66

Dole VP, Nyswander ME (1965) A Medical Treatment for diacetylmorphine (heroin-) addiction. JAMA $\underline{193}, 646-650$

Dollard J, Miller N: Personality and Psychotherapy. Mc Graw-Hill, New York 1950.

Driessen M, Schulte S, Luedecke C, Schaefer I, Sutmann F, Ohlmeier M, Kemper U, Koesters G, Chodzinski C, Schneider U, Broesse T, Dette C, HavemannReinecke U, Traumab-Study Group (2008): Trauma and PTSD in patients with alcohol, drug, or dual dependence: a multi-center study. Alcohol Clin Exp Res $\underline{32}$ (3), 481-488

Drogenbeauftragte der Bundesregierung: Drogen- und Suchtbericht Mai 2011; Bundesministerium für Gesundheit, Berlin 2011

Drogenbeauftragte der Bundesregierung: Drogen- und Suchtbericht Mai 2012; Bundesministerium für Gesundheit, Berlin 2012

Dube SR, Anda RF, Felitti VJ, Chapman DP, Williamson DF, Giles WH (2001): Childhood abuse, household dysfunction, and the risk of attempted suicide throughout the life span: findings from the Adverse childhood Experiences Study. JAMA 286, 3089-3096

Ducan RD, Saunders BE, Kilpatrick DG, Hanson RF, Resnick HS (1996): Childhood physical assault as a risk factor for PTSD, depression and substance abuse: Findings from a national survey. Am J Orthopsychiatry $\underline{66}, 437-448$

Eisenbach-Stangl I, Uhl A: Geschichte und Praxis der Substitutionsbehandlung in Österreich; in Methadonbehandlung. Ein Leitfaden, hrsg. v. Bossong H, Stöver H; Campus, Frankfurt 1992, 181-197

Eisler R M, Hersen M, Agras W St (1973): Effects of videotape and instructional feedback on nonverbal marital Interaction. An analog study. Behav Ther $\underline{4}, 551$ 558 
Ekblad S, Manicavasagar V, Silove D, Baarnhielm S, Reczycki M, Mollica R, Coello M (2004): The use of international videoconferencing as a strategy for teaching medical students about transcultural psychiatry. Transcult Psychiatry $\underline{41}$ (1), 120-129

Elman I, Karlsgodt KH, Gastfriend DR (2001): Gender Differences in Cocaine Craving Among Non-Treatment-Seeking Individuals with Cocaine Dependence. Am J Drug Alcohol Abuse $2 \underline{7}$ (2), 193-202

Engel CC, Engel AL, Campbell SI, McFall ME, Russo J, Katon W (1993): Posttraumatic stress disorder symptoms and precombat sexual and physical abuse in Desert Storm veterans. J Nerv Ment Dis $\underline{181}$ (11), 683-688

Epstein DH, Preston KL (2003): Does Cannabis use predict poor outcome for heroin dependent patients on maintenance treatment? Past findings and more evidence against. Addiction $\underline{98}$ (3), 269-279

Esse S, Schulz W, Wild A: Evaluierung des qualifizierten Drogenentzugs im Niedersächsischen Landeskrankenhaus Königslutter, in: Schriftenreihe der Suchtforschungsstelle Ostniedersachsen, Heft 22; hrsg. v. Mauthe J-H, Schulz W; Braunschweig 1994, 1-93

Feinstein J S, Tamerin J S (1972): Induced intoxication and videotapefeedback in alcoholism treatment. J Stud Alcohol $\underline{33}, 408-416$

Festinger L: A Theory of Cognitive Dissonance. Stanford University Press, Stanford, CA 1957

Finkelhor TJ (1994): The international epidemiology of child sexual abuse. Child Abuse Negl $\underline{18}$ (5), 409-417

Franken IH, Hendriks VM (1999): Predicting outcome of inpatient detoxification of substance abusers. Psychiatr Serv $\underline{50}$ (6), 813-817

Furman S, Feighner A (1973): Videofeedback in treating hyperkinetic children: a preliminary report. Am J Psychiatry $\underline{130}$ (7), 792-796

Gallimberti L, Cibin M, Pagnin P, Sabbion R, Pani PP, Pirastu R, Ferrara SD, Gessa GL (1993): Gamma-hydroxybutyric acid for treatment of opiate withdrawal syndrome. Neuropsychopharmacology $\underline{9}(1), 77-81$

Gastpar M, Rösinger C (1996): Therapie der Opiatabhängigkeit. Dtsch Arztebl $\underline{93}$ (5), A 249-251

Gearing FR (1970): Evaluation of methadone maintenance treatment program. Int J Addict $\underline{5}$ (3), 517-543 
Geertsma R S, Reivich R S (1965): Auditory and visual dimensions of

externally mediated self-observation. J Nerv Ment Dis $\underline{148}, 210-223$

Giaconia RM, Reinherz HZ, Silverman AB, Pakiz B, Frost AK, Cohen E (1995): traumas and posttraumatic stress disorder in a community population of older adolescents. J Am Acad Child Adolesc Psychiatry 34, 1369-1380

Gößling HW (1997): Klinischer Drogenentzug im Schnittpunkt zwischen akzeptierenden und abstinenzorientierten Behandlungsstrategien. Sozialpsychiatr Inf $\underline{2}$, 69

Gößling HW, Sorge J, Werry C, (1997): Der Turbo-Entzug - bequem schneller clean? Möglichkeiten und Grenzen des narkosegestützten Opiatschnellentzugs (FORNO). Niedersachs Arztebl (71) 9, 32-33

Gößling HW, Gunkel S, Wegener U. Woiton V (1998): Auswirkungen fraktionierter Methadonangabe im klinischen Opiatentzug. Fortschr Neurol Psychiatr $\underline{66}$ (6), 278-285

Gößling HW, Gunkel S, Schneider U, Melles W (2001): Häufigkeit und Bedingungsfaktoren des Behandlungsabbruchs im stationären Drogenentzug. Fortschr Neurol Psychiatr $\underline{69}$ (10), 474-481

Goldfield M D, Levy R ( 1968): The use of television videotape to enhance the therapeutic value of psychodrama. Am J Psychiatry 125 (5), 690-692

Gossop M, Strang J (1991): A comparison of the withdrawal responses of heroin and methadone addicts during detoxification. Br J Psychiatry 158, 697-699

Gossop M, Griffiths P, Bradley B, Strang J (1989): Opiate withdrawal symptoms in response to 10-day and 21-day methadone withdrawal programmes. Br J Psychiatry $\underline{154}, 360-363$

Gossop M, Marsden J, Stewart D (2006): Remission of Psychiatric Symptoms Among Drug Misusers After Drug Dependence Treatment. J Nerv Ment Dis 194 (11), 826-832

Grant BF, Stinson FS, Dawson DA, Chou SP, Dufour MC, Compton W, Pickering RP, Kaplan K (2004): Prevalence and co-occurrence of substance use disorders and independent mood and anxiety disorders: results from the national Epidemiologic Survey on Alcohol and Related Conditions. Arch Gen Psychiatry $\underline{61}$ (8), 807816

Green CA (2006): Gender and Use of Substance Abuse Treatment Services. Alcohol Res Health $\underline{29}$ (1), 55-62

Griffiths RD (1974): Videotape Feedback as a therapeutic technique: Retrospect and prospect. Behav Res Ther 12 (1), 1-8 
Griffiths R D, Hinkson J (1973): The effects of videotape feedback on the self assessments of psychiatric patients. Br J Psychiatry 123 (573), 223-224

Grönbladh L, Ohlund LS, Gunne LM (1990) : Mortality in heroin addiction: impact of methadone treatment. Acta Psychiatr Scand $\underline{83}$ (3), 223-227

Günther A, Dedner C, Schäfer G, Berl J, Kuder T, Bader T, Dengler W (2000): Komorbidität bei Drogenabhängigen. Empirische Ergebnisse und therapeutische Konsequenzen. Suchttherapie 1, 16-20

Gunne LM (1983): The case of the Swedish methadone maintenance treatment program. Drug Alcohol Depend 11 (1), 99-103

Gutjahr E, Gmel G, Rehm J (2001): Relation between average alcohol consumption and disease: An overview. Eur Addict Res $\underline{7}, 117-127$

Handmaker N S, Hester R K, Delaney H D (1999): Videotaped training in alcohol counselling for obstetric care pracitioners: A randomized controlled trial. Obstet Gynecol $\underline{93}(2), 213-218$

Harden C L, Burgut F T, Kanner A M (2003): The diagnostic significance of videoEEG monitoring findings on pseudoseizure patients differs between neurologists and psychiatrists. Epilepsia 44 (3), 453-456

Harris EC, Barraclough B (1997): Suicide as an outcome for mental disorders. A meta-analysis. Br J Psychiatry 170, 205-228

Hartel DM, Schoenbaum EE, Selwyn PA, Kline J, Davenny K, Klein RS, Friedland GH (1995): Heroin Use during Methadone Maintenance Trreatment: The Importance of Methadone Dose and Cocaine Use. Am J Public Health $\underline{85}$ (1), 83-88

Harvey A G, Clark D M, Ehlers A, Rapee R M (2000): Social anxiety and selfimpression: cognitive preparation enhances the beneficial effects of video feedback following a stressful social task. Behav Res Ther $\underline{38}$, 1183-1192

Havard A, Teesson M, Darke S, Ross J (2006): Depression among heroin users: 12-Month outcomes from the Australian Treatment Outcome Study (ATOS). J Subst Abuse Treat $\underline{30}$ (4), 355-362

Helander A, von Wachenfeldt J, Hiltunen A, Beck O, Liljeberg P, Borg S (1999): Comparison of urinary 5-hydroxytryptophol, breath ethanol, and self-report for detection of recent alcohol use during outpatient treatment: A study on methadone patients. Drug Alcohol Depend $\underline{56}(1), 33-38$

Helzer JE, Robin L N, McEnvoy L (1987): Post-traumatic stress disorder in the general population. N Engl J Med 317, 1630-1634 
Hirsch D, Tretter F (2000): Einflussfaktoren für Therapieabbrüche beim qualifizierten Drogenentzug. Suchtmed Forsch Prax $\underline{2}$ (1), 31-37

Hoffmann M, Weithmann G, Grupp D, Rothenbacher H, Kapp B, Mann K (1997): Erreichen niederschwellige Entzugsstationen neue Gruppen von Drogenabhängigen? Psychiatr Prax $\underline{24}$ (6), 296-299

Hornstein C, Schenk S, Wortmann-Fleischer S, Downing G, Schwarz M (2006) Videotherapie bei postpartalen Störungen. Psychotherapeut $\underline{5}, 363-368$

Hulse GK, English DR, Milne E, Holman CDJ (1999): The quantification of mortality resulting from the regular use of illicit opiates. Addiction $\underline{94}$ (2), 221-229

Hunt DE, Strug DL, Goldsnith DS, Lipton DS, Robertson K, Truitt L (1986): Alcohol use and abuse: Heavy drinking among methadone clients. Am $\mathrm{J}$ Drug Alcohol Abuse 12 (1-2), 147-164

Hunt D P (1964): The effects of nonlinear and discrete transformations of feedback information on human tracking performance. J Exp Psychol 67, 486-494

Iguchi M, Handelsman L Bickel W, Griffiths R (1993): Benzodiazepine and sedative use/abuse by methadone maintenance clients. Drug Alcohol Depend $\underline{32}$ (3), 257-266

Janis IL: Effects of fear arousal on attitude change: Recent developments in theory and experimental research; in: Advances in experimental social psychology, vol. 3; hrsg. v. Berkowitz L; Academic Press, New York 1967, 166-224

Jeremiah J, O’Sullivan P, Stein MD (1995): Who leaves against medical advice. J Gen Intern Med 10 (7), 403-405

Johanson A, Risberg J, Tucker D M, Gustafson L (2006): Changes in frontal lobe activity with cognitive therapy for spider phobia. Appl Neuropsychol $\underline{13}$ (1), 34-41

Jones P: The technique of the television camera. 3. Auflage; Focal Press, London 1972

Kalbag AS, Levin FR (2005): Adult ADHD and Substance Abuse: Diagnostic and Treatment Issues. Subst Use Misuse $\underline{40}$ (13-14), 1955-1981

Kang H, Dalager N, Mahan C, Ishii E (2005): The role of sexual assault on the risk of PTSD among Gulf War veterans. Ann Epidemiol 15 (3), 191-195

Kanof PD, Aronson MJ, Ness R (1993): Organic mood syndrome associated with detoxification from methadone maintenance. Am J Psychiatry 150 (3), 423-428 
Kemner C, Klein M, Zemlin U (2004): Gewalterfahrungen bei Patientinnen und Patienten in einer Fachklinik für Alkoholabhängige: Ergebnisse einer Prävalenzerhebung und Konsequenzen für das Behandlungsprogramm. Suchttherapie $\underline{5,}$ 124131

Kendler KS, Bulik CM, Silberg J, Hettema JM, Prescott CA (2000): Childhood sexual abuse and adult psychiatric and substance use disorders in women: an epidemiological and cotwin control analysis. Arch Gen Psychiatry 57, 653-959

Kessler RC, McGonagle KA, Zhao S, Nelson CB, Hughes M, Eshlman S, Wittchen H U, Kendler KS (1994): Lifetime and 12-month prevalence of DSM-III-R psychiatric disorders in the United States. Results from the national Comorbidity Survey. Arch Gen Psychiatry $\underline{51}$ (1), 8-19

Kessler RC, Sonnerga A, Bromet E, Hughes M, Nelson CB (1995): Posttraumatic stress disorder in the National Comorbidity survey. Arch Gen Psychiatry $\underline{52}$ (12), 1048-1060

Kessler RC, Nelson CB, McGonagle KA, Edlund MJ, Frank RG, Leaf PJ (1996): The epidemiology of co-occurring addicitive and mental disorders: Implications for prevention and service utilization. Am J Orthopsychiatry; $\underline{66}$ (1), 17-31

Kilpatrick D, Acierno R, Resnick H, Saunders B, Best C (1997): A 2-year longitudinal analysis of the relationships between violent assault and substance use in woman. J Consult Clin Psychol $\underline{65}$ (5), 834-847

Kim H Y, Lundh L-G, Harvey A (2002): The enhancement of videofeedback by cognitive preparation in the treatment of social anxiety. A single-session experiment. J Behav Ther Exp Psychiatry $\underline{33}$ (1), 19-37

Kipp J, Stolzenburg HJ (2000): Sucht und Abstinenz im Zusammenhang mit der Aktivierung früher Persönlichkeitsstrukturen. Psychotherapeut_45, 32-38

König S, Wodarz N, Unglaub W, Lohann M (2007) Aufmerksamkeitsdefizit/ Hyperaktivitätssyndrom (ADHS) bei erwachsenen Drogenabhängigen. Psychiatr Prax $\underline{34}$ Suppl 1, 71-72

Krach C, Peschke H (1987): Das Hannoversche Methadonprogramm - 11 Jahre danach. Manuskript Hannover (erwähnt bei Hüsgen HA (2009): Über die Entwicklung der Substitution bei opiatabhängigkeit in der medizinischen Rehabilitation. Suchttherapie 10 (4), 180-185

Kraus L, Augustin R, Orth B (2005): Illegale Drogen, Einstiegsalter und Trends. Ergebnisse des Epidemiologischen Suchtsurvey 2003. Sucht $\underline{51}$ (Sonderheft 1), $19-28$

Krausz M (1995): Sucht in der psychiatrischen Praxis - Neue Tendenzen in der Suchttherapie. Psychiatr Prax 22 (5), 177-178 
Krausz M (2004): Was, wie erreichen und warum? Welche Paradigmen steuern die Suchttherapie der Zukunft? Suchttherapie $\underline{5}, 16-20$

Krausz M, Briken P (2002): Sexueller Missbrauch bei opiatabhängigen Frauen in Relation zu biografischen Faktoren, Suchtentwicklung und psychischer Symptomatik. Suchttherapie $\underline{3}$, 178-183

Krausz M, Verthein U, Dengkwitz P (1998): Prävalenz psychischer Störungen bei Opiatabhängigen mit Kontakt zum Drogenhilfesystem. Nervenarzt $\underline{69}$ (2), 557-567

Kreyssig U: Zum Zusammenhang von sexuellem Missbrauch und Sucht; in: Sexueller Missbrauch. Überblick zur Forschung, Beratung und Therapie. Ein Handbuch; hrsg. v. Amann G, Wipplinger R; dgvt-Verlag, Tübingen 1997, 260-273

Küfner H (1995): Behandlungsfaktoren bei Alkohol- und Drogenabhängigen. Z Klin Psychol Psychother 24 (2), 170-183

Kuntze M, Ladewig D, Stohler R (1998): Art und Häufigkeit der Komorbidität bei methadonsubstituierten Opiatabhängigen in der ambulanten Versorgung. Sucht 44 (2), 96-103

Kutscher S, Hayatghebi S, Birkhahn H, Thiemeier R, Reinert T, Tagay S, Langkafel M, Banger M (2002): Traumatische Lebensereignisse und posttraumatische Belastungsstörungen bei alkoholabhängigen Patienten. Nervenarzt 1, Suppl. 200201

Lairson D, Swint J (1978): A multivariate analysis of the likelihood and volume of preventive demand in a prepaid group practice. Med Care 16, 730-739

Landolt H P, Glatzel M, Blattler T, Achermann P, Roth C, Mathis J, Weis J, Tobler I, Aguzzi A, Bassetti C L (2006): Sleep-wake disturbances in sporadic CreutzfeldtJakob disease. Neurology $\underline{66}(9), 1418-1424$

Latowsky M (1996): Improving detoxification outcomes from methadone maintenance treatment: the interrelationship of affective states and protracted withdrawal. J Psychoactive Drugs $\underline{28}$ (3), 251-157

Leri F, Bruneau J, Stewart J (2003): Understanding polydrug use : review of heroin and cocaine co-use. Addiction $\underline{98}$ (1), 7-22

Leventhal $\mathrm{H}$ : Findings in theory in the study of fear communications; in: Advances in experimental social psychology, Band 5; hrsg. v. Berkowitz L; Academic Press, New York 1970,119-186

Leventhal H, Hirschman R S: Social psychology and prevention; in: Social Psychology of health and illness; hrsg. v. Sanders GS, Suls JM; Lawrence Erlbaum, Hillsdale, NJ 1982, 183-226 
Leventhal H, Watts JS (1966): Sources of resistance to fear-arousing communications on smoking and lung cancer. J Pers $\underline{34}$ (2), 155-175

Liese BS, Beck AT (1997): Back to the basics: Fundamental cognitive therapy skills for keeping drug-dependent individuals in treatment. NIDA Res Monogr $\underline{165}$, 233-244

Lincoln RS (1956): Learning and retaining a rate of movement with the aid of kinesthetic and verbal causes. J exp Psychol 51, 199-204

Linehan MM: Dialektisch-Behaviorale Therapie der BorderlinePersönlichkeitsstörung. CIP-Medien, München 1996 a

Linehan MM: Trainingsmanual zur Dialektisch-Behavioralen Therapie der Borderline-Persönlichkeitsstörungen. CIP-Medien, München 1996 b

Loimer N (1992): Vier Jahre Methadonsubstitutionstherapie in Österreich. Wien klin Wochenschr 104 (19), 583-588

Loimer N, Linzmayer L, Schmid R, Grunberger J (1991): Similar efficacy of abrupt and gradual opiate detoxification. Am J Drug Alcohol Abuse 17 (3), 307-312

Loken B, Howard-Pitney B (1988): Effectiveness of cigarette advertisements on women: an experimental study. J Appl Psychol $\underline{73}$ (3), 378-382

Longwell B, Betz T, Horton H, Witte CL, Witte MH (1979): Weight gain and edema on methadone maintenance therapy. Int J Addict 14 (3), 329-335

Lüdecke C, Sachsse U, Faure H: Sucht - Bindung - Trauma Psychotherapie von Sucht und Traumafolgen im neurobiologischen Kontext. Schattauer, Stuttgart 2010

MacKinnon D P, Nemeroff C, Nohre L (1994): Avoidance responses to alternative alcohol warning labels. J Appl Soc Psychol 24, 733-753

Maddux J E, Rogers R W (1983): Protection motivation and self efficacy: A revised theory of fear appeals and attitude change. J Exp Soc Psychol 19, 469-479

Madelung E, Dunkel D, Haring C (2006): Substitutionsmittel und Beikonsum bei Opiatabhängigen. Suchttherapie $\underline{7}, 18-23$

Marlowe DB, Husband SD, Bonieski LM, Kirby KC, Platt JJ (1997): structured interview versus self-report test vantages for the assessment of personality pathology in cocaine dependence. J Personal Disord 11 (2), 177-190

Marsch LA (1998): The efficacy of methadone maintenance interventions in reducing illict opiate use, HIV risk behaviour and criminality: a metaanalysis. Addiction 93 (4), 515-532 
Maser JD, Patterson P (2002): Spectrum and nosology: implications for DSM-V. Psychiatr Clin North Am $\underline{25}$ (4), 855-885

Mattick RP, Breen C, Kimber J, Davoli M (2003): Methadone maintenance therapy versus no opioid replacement therapy for opioid dependence. Cochrane Database Syst Rev (2), CD 002209

McCaul ME, Stitzer MC, Bigelow GE, Liebson IA (1984): Outpatient methadone detoxification: effects of diazepam and doxepin as adjunct medication. NIDA Res Monogr $\underline{55}, 191-196$

McCauley J, Kern DE, Kolodner K, Dill L, Schroeder AF, DeChant HK, Ryden J, Derogatis LR, Bass EB (1997): Clinical characteristics of women with a history of childhood abuse: unhealed wounds. JAMA $\underline{277}$ (17), 1362-1368

McGuigan F J (1959): The effects of precision, delay and schedule of knowledge of results on performance. $\mathrm{J}$ exp Psychol $\underline{58}, 79-84$

McGuire W J: Personality and suspectibility to social influence; in: Handbook of personality theory and research; hrsg. v. E. F. Borgatta EF, Lambert WW; Rand McVally, Chicago 1968 a, 1130-1187

McGuire W J: Personality and attitude change: An information processing theory; in: Psychological foundations of attitudes; hrsg. v. Greenwald AG, Brook TC, Ostrom TM; Academic Press, New York 1968 b, 171-196

McGuire W J: An information-processing model of advertising effectiveness; in: Behavioural and management science in marketing; hrsg. v. Davis HJ, Silk AJ, Wiley, New York 1978

McLellan AT, Luborsky L, Woody GE, O'Brien CP, Druley KA (1983) Predicting response to alcohol and drug abuse treatments. Role of psychiatric severity. Arch Gen Psychiatry 40 (6), 620-625

McLellan AT, Grissom GR, Zanis D, Randall M, Brill P, O’Brien CP (1997) : Problem-service 'matching' in addiction treatment. A prospective study in 4 programs. Arch Gen Psychiatry 54 (8), 730-735

Meier PS, Barrawclough C, Donmall MC (2005 a): The role of the therapeutic alliance in the treatment of substance misuse: A critical review of the literature. Addiction $\underline{100}, 304-316$

Meier PS, Donmall MC, Barrawclough C, McElduff P, Heller RF (2005 b): Predicting the early therapeutic alliance in the treatment of drug misuse. Addiction $\underline{100}$, 500-511

Meili D, Dober S, Eyal E (2004): Jenseits des Abstinenzparadigmas - Ziele in der Suchttherapie. Suchttherapie $\underline{5}, 2-9$ 
Milby JB, Hohmann AA, Gentile M, Huggins N, Sims NK, Mc Lellan AT, Woody G, Haas N (1994): Methadone maintenance outcome as a function of detoxification phobia. Am J Psychiatry 151 (7), 1031-1037

Miller WR, Rollnick S: Motivierende Gesprächsführung: Ein Konzept zur Beratung von Menschen mit Suchtproblemen. Lambertus, Freiburg im Breisgau 1999

Mills KL, Lynsky M, Teesson M, Ross J, Darke S (2005): Post-traumatic stress disorder among people with heroin dependence in the Australian treatment outcome study (ATOS): prevalence and correlates. Drug Alcohol Depend 77, 243-249

Ministerium für Arbeit, Gesundheit und Soziales des Landes Nordrhein-Westfalen (Hrsg): Medikamentengestützte Rehabilitation i.v. Opiatabhängiger. Katamnese. Prognos GmbH, Köln 1998

Moner SE (1996): Acupuncture and addiction treatment. J Addict Dis 15 (3), 79100

Moore F J, Chernell E, West M J (1965): Television as a therapeutic tool. Arch Gen Psychiatry (Chicago) 12, 217-220

Mullen PE, Martin JL, Anderson JC, Romans SE, Herbison GP (1993): Childhood sexual abuse and mental health in adult life. Br J Psychiatry 163, 721-732

Nasseri K (1979): Letters to the editor. Int J Epidemiol $\underline{8}$ (4), 389-390

Neumann M, Kasper S, Krappel C, Möller HJ, Laux G, Paul HW (2002): Niederschwelliger Drogenentzug - Ergebnisse eines Modellprojekts. Krankenhauspsychiatrie $\underline{13}, 99-103$

Newton-Taylor B, De Wit D, Gliksman L (1998): Prevalence and factors associated with physical and sexual assault of female university students in Ontario. Health Care Women Int $\underline{19}$ (2),155-164

North CS, Nixon SJ, Shariat S, Mallonee S, McMillen JC, Spitznagel EL, Smith EM (1999): Psychiatric disorders among survivors of the Oklahoma City bombing. JAMA 282, 755-762

O’Brien CP, Charney DS, Lewis L, Cornish JW, Post RM, Woody GE, Zubieta JK, Anthony JC, Blaine JD, Bowden CL (2004): Priority actions to improve the care of persons with co-occurring substance abuse and other mental disorders: a call to action. Biol Psychiatry $\underline{56}, 703-713$

O'Brien JS, Raynes AE, Patch VD (1972): Treatment of heroin addiction with aversion therapy, relaxation training and systematic desensitization. Behav Res Ther $\underline{10}$ (1), $77-80$ 
Paetzold W, Eronat V, Seifert J, Holze E, Emrich HM, Schneider U (2000): Detoxifikation polytoxikomaner Patienten mit Buprenorphin. Nervenarzt $\underline{71}$ (9), 722-729

Paredes A, Ludwig K D, Hassenfeld I N, Cornelison F S (1969): A clinical study of alcoholics using audiovisual self-image feedback. J Nerv Ment Dis 148 (4), 449456

Pelissier BM, CampSD, Gaes GG, Saylor WG, Rhodes W (2003) : Gender differences in outcomes from prison-based residental treatment. J Subst Abuse Treat 24, $149-160$

Petry NM, Bickel WK (1999): Therapeutic alliance and psychiatric severity as predictors of contempletion of treatment for opioid dependence. Psychiatr Serv $\underline{50}$ (2), 219-227

Petry NM, Bickel WK (2000): Gender differences in hostility of opioid dependent outpatients: role in early treatment termination. Drug Alcohol Depend 58, 27-33

Petty RE, Cacioppo JT (1986): The elaboration likehood model of persuasion. Adv Exp Soc Psychol 19, 123-205

Petty RE, Baker SM, Gleicher F : Attitudes and drug abuse prevention : Implications of the elaboration likehood model of persuasion ; in : persuasive communication and drug abuse prevention; hrsg. v. Donohew L, Sypher E, Bukoski WJ; Lawrence Erlbaum Associates, Inc., Hillsdale, NJ 1991, 71-90

Pfab R, Hirtl C, Hibler A, Felgenhauer N, Chlistalla J, Filker T (1996): Der Antagonistinduzierte Narkose-gestützte Opiat-Schnellentzug (AINOS). MMW Munch Med Wochenschr $\underline{138}$, 781-786

Platt JJ, Husband SD, Taube D (1990): Major psychotheraeutic Modalities for heroin addiction: a brief overview. Int J Addict 25 (12A), 1453-1477

Preuss UW, Barnow S, Zimmermann J, Watzke AB, Schultz G, Wie MW (2004): Suizidalität bei Abhängigkeitserkrankungen: Eine Übersicht. Suchtmed Forsch Prax $\underline{6}$ (4), 271-285

Probst M, van Coppenolle H, Vandereycken W, Meermann R (1990): Evaluation of body image perception in patients with anorexia nervosa. Psychiatr Prax 17 (3), 115-120

Rapee R M, Hayman K (1996): The effects of video feedback on the selfevaluation of performance in socially anxious subjects. Behav Res Ther 34 (4), 315-322

Raschke P: Substitutionstherapie. Ergebnisse langfristiger Behandlung von Opiatabhängigen. Lambertus, Freiburg i. Br. 1994 
Raschke P Schliehe F, Fischer D, Groenemeyer A: Therapie und Rehabilitation bei Drogenkonsumenten. Ministerium für Arbeit, Gesundheit und Soziales NRW (Hrsg), Düsseldorf 1985

Raskin VD, Miller NS (1993): The epidemiology of the comorbidity of psychiatric and addictive disorders: a critical review. J Addict Dis 12 (3), 45-57

Rasting M, Borsig B, Beutel M E (2005): Alexithymic characteristics and patienttherapist interaction: a video analysis of facial affect display. Psychopathology $\underline{38}$ (3), 105-111

Regier DA, Farmer ME, Rae DS, Locke BZ, Keith SJ, Judd LL, Goodwin FK (1990): Comorbidity of mental disorders with alcohol and other drug abuse. Results from the Epidemiologic Catchment Area (ECA) Study. JAMA 264 (19), 25112518

Roch I, Küfner H, Arzt J, Böhmer M, Denis A (1992): Empirische Ergebnisse zum Therapieabbruch bei Drogenabhängigen: Ein Literaturüberblick. Sucht 38 (5), 304322

Rogers R W (1975): A protection motivation theory of fear appeals and attitude change. J Psychol $\underline{\text { 91, 93-114 }}$

Rogers R W: Cognitive and physiological processes in fear appeals and attitude change: a revised theory of protection motivation; in: Social psychophysiogy: A source-book; hrsg. v. Cacioppo JT, Petty RE; Guilford Press, New York 1983, 153176

Rogers RW, Prentice-Dunn S: Protection Motivation Theory; in: Handbook of Health Behaviour Research: Personal and Social Determinants; hrsg. v. Gochman DS; Vol. 1; Plenum Press, New York 1997,113-123

Roscher D: Untersuchung zur Validierung des Video-Assistierten Monitoring therapeutischer Prozesse. Med. Diss. Göttingen 2006

Rosenbaum M (1991): Staying off methadone maintenance. J Psychoactive Drugs $\underline{23}$ (3), $251-260$

Rosenberg MJ, Hovland Cl: Cognitive, Affective, and Behavioral Components of Attitudes; in: Attitude organization and change; hrsg.v. Hovland Cl, Rosenberg MJ; Yale University Press, New Haven CT 1960, 1-14

Rössler H, Battista HJ, Riemer Y, Neumann B, Unterdorfer H, Wehinger G, Henn R, Hinterhuber H (1993): Todesfälle von Drogensüchtigen - Eine kritische Analyse. Nervenarzt $\underline{64}, 599-605$ 
Rounsaville BJ, Weissman MM, Kleber HD (1986): Prognostic significance of psychopathology in treated opiate addicts. A 2,5 year follow-up study. Arch Gen Psychiatry $\underline{43}$ (8) $739-745$

Rounsaville BJ, Kleber HD (1985): Psychotherapy/ Counseling for opiate addicts: strategies for use in different treatment settings. Int J Addict $\underline{20}$ (6-7), 869-896

Rüesch P, Hättenschwiler J (2002): Konsequenzen von Rückfall und behandlungsabbruch im stationären Drogenentzug: eine 1-Monats-Katamnese. Schweiz Arch Neurol Psychiatr 153 (5), 238-244

Safren SA, Gershuny BS, Marzol P, Otto MW, Pollack MH (2002): History of childhood abuse in panic disorder, social phobia, and generalized anxiety disorder. $\mathrm{J}$ Nerv Ment Dis 190 (7), 453-456

Salter AC: Treating child sex offender. A practical guide. Sage, London 1988

San L, Cami J, Peri JM, Mata R, Porta M (1989): Success and failure at inpatient heroin detoxification. $\mathrm{Br} \mathrm{J}$ Addict $\underline{84}$ (1), 81-87

Schaefer H H, Sobell M B, Mills K C (1971): Some sobering data on the use of self confrontation with alcoholics. Behav Ther $\underline{2}$, 28-39

Schäfer M, Schnack B, Soyka M (2000): Sexueller und körperlicher Missbrauch während der frühen Kindheit oder Adoleszenz bei späterer Drogenabhängigkeit. Psychother Psychosom Med Psychol $\underline{50}$ (2), 38-50

Schanda H, Presslich O, Hermann P (1983): Clonidin zur Behandlung des Heroinentzugssyndroms. Wien Klin Wochenschr 95 (16), 569-572

Scherbaum N, Klein S, Kaube H, Kienbaum P, Peters J, Gastpar M (1998): Alternative strategies of opiate detoxification: evaluation of the so-called utra-rapid detoxification. Pharmacopsychiatry $\underline{31}$ (6), 205-209

Schmidt SA: Prävalenz sexuellen Kindesmissbrauchs bei Opiatabhängigen Themenbezogene Grundlagen, Konzepte, Durchführung und Ergebnisse eines Kontrollgruppenvergleichs. Verlag für Wissenschaft und Bildung, Berlin 2000

Schnieders M, Rassaerts I, Schäfer M, Soyka M (2006): Der Einfluss kindlicher Traumatisierung auf eine spätere Drogenabhängigkeit. Fortschr Neurol Psychiatr $\underline{74}, 511-521$

Schulz W, Wild A, Esse S: Evaluierung des qualifizierten Drogenentzugs im Niedersächsischen Landeskrankenhaus Osnabrück; in: Schriftenreihe der Suchtforschungsstelle Ostniedersachsen; Heft 24; hrsg. v. Mauthe J-H, Schulz W, Braunschweig 1995, 1-83 
Schwarz N: Feelings as information: Informational and motivational functions of affective states; in: Handbook of motivation and cognition: Foundations of social behaviour, vol 2; hrsg.v. Sorrentino RM, Higgins ET; Guilford Press, New York 1990, 527-561

Schwarz N, Bless H: Happy and mindless, but sad and smart? Impact of affective states on analytic reasoning; in: Emotion and social judgments; hrsg. V. Forgas J; Pergamon, Oxford 1991, 55-71

Schwarzer R (1993): Defensiver und funktionaler Optimismus als Bedingungen für Gesundheitsverhalten. Z Gesundheitspsychol 1 (1), 7-31

Schwarzer R (1994): Optimism, vulnerability, and self-beliefs as health-related cognitions: a systemic overview. Psychol Health $\underline{9}, 161-180$

Sees KL, Delucchi KL, Masson C, Rosen A, Clark HW, Robillard H, Banys P, Hall SM (2000): Methadone maintenance vs 180-day psychosocially enriched detoxification for treatment of opioid dependence: a randomized controlled trial. JAMA $\underline{283}$ (10), 1303-1310

Segest E, Mygind O, Bay H (1990): The influence of prolonged Stable methadone maintenance treatment on mortality and employment: An 8-year follow-up. Int $\mathrm{J}$ Addict 25 (1); 53-63

Senay EC, Dorus W, Showalter C (1984): Methadone detoxification: self versus physician regulation. Am J Drug Alcohol Abuse 10 (3), 361-374

Seppä K, Aalto M, Raevaara L, Peräkylä A (2004): A brief intervention for risky drinking-analysis of videotaped consultations in primary health care. Drug Alcohol $\operatorname{Rev} \underline{23}(2), 167-170$

Shapiro F, Vogelmann-Sine S, Sine LF (1994): Eye movement desensitisation and reprocessing: treating trauma and substance abuse. J Psychoactive Drugs $\underline{26}$ (4), 379-391

Silvermann AB, Reinherz HZ, Giaconia RM (1996): The long-term sequelae of child and adolescent abuse: A longitudinal community study. Child Abuse Negl $\underline{20,}$ 709-724

Simons HW: Persuasion: Understanding, Practice, and Analysis. 2. Auflage; McGraw-Hill, Inc., New York 1986

Simpson TL, Miller WR (2002): Concomitance between childhood sexual and physical abuse and substance use problems. A review. Clin Psychol Rev 22, 2777

Sonntag D, Hellwich AK, Bauer C (2007): Deutsche Suchthilfestatistik 2006 für stationäre Einrichtungen. Sucht $\underline{53}, 42-64$ 
Southwick-Bensley L, Wu R (1991): The rule of psychological reactance in drinking following alcohol prevention messages. J Appl Soc Psychol 21, 1111-1124

Sporer KA (2003): Strategies for preventing heroin overdose. Br J Med 326 (7386), 442-444

Staak M, Rupp C (1993): Lebenserwartung des Drogenkonsumenten. Versicherungsmedizin $\underline{45}, 10-14$

Stainback RD, Rogers (1983) Identifying effective components of alcohol abuse prevention programms: Effect of fear appeals, message style, and source expertise. Int J Addict 18, 393-405

Stastney D, Potter M (1991) Alcohol abuse by patients undergoing methadone treatment programmes. Br J Addict $\underline{86}, 307-310$

Stein MB (2002): A 46-year-old man with anxiety and nightmares after a motor vehicle collision. JAMA 288 (12), 1513-1522

Stein A, Woolley H, Senior R, Hertzmann L, Lovel M, Lee J, Cooper S, Wheatcroft R, Challacombe F, Patel P, (2006): Treating disturbance in the relationship between mothers with bulimic eating disorders and their infants: a randomized, controlled trial of video feedback. Am J Psychiatry 163 (5), 899-906

Stoller F H (1969): Videotape Feedback in the Group setting. J Nerv Ment Dis 148 (4), 457-466

Strang J, Marks I, Dawe S, Powell J, Gossop M, Richards D, Gray J (1997): Type of hospital setting and treatment outcome with heroin addicts. Results from a randomised trial. Br J Psychiatry 171, 335-339

Stuppe M (2002): Dialektisch-Behaviorale Therapie bei Suchtkranken mit schweren Persönlichkeitsstörungen. Suchttherapie $\underline{3}, 241-245$

Subkowski P (2000): Die psychoanalytische Sicht der Abhängigkeitserkrankungen. Psychotherapeut $\underline{45}, 253-266$

Sutton S (1982): Fear arousing communications: A critical examination of theory and research; in: Social Psychology and behavioural medicine; hrsg. v. Eiser JR; John Wiley and Sons, New York 1982, 303-337

Sutton S, Eiser J R (1984): The effect of fear-arousing communications on cigarette smoking: An expectancy-value approach. J Behav Med $\underline{7}$ (1), 13-33

Sutton S, Hallet R (1989): The contribution of fear and cognitive factors in mediating the effects of fear arousing communications. Social Behavior $\underline{4}, 83-98$ 
Teegen F, Zumbeck S (2000): Prävalenz traumatischer Erfahrungen und Posttraumatischer Belastungsstörung bei substanzabhängigen Personen. Psychotherapeut $\underline{45}, 44-49$

Trafton JA, Minkel J, Humphreys K (2006): Opioid Substitution Treatment Reduces Substance Use Equivalently in Patients With and Without Posttraumatic Stress Disorder. J Stud Alcohol $\underline{67}$ (2), 228-235

Tretter F: Methadonsubstitution: Zur Problematik der zusätzlichen Verordnung von Benzodiazepinen. Diskussionspapier Bayrische Akademie für Suchtfragen e.V., München 2001, 1-8

Triandes H C: Attitude and attitude change. Wiley, New York 1971

Trüg E (1992): Evaluationsstudie zum niederschwelligen Drogenentzug opiatabhängiger Patienten in Hamburg - eine empirische Studie. Sucht $\underline{38}$, 323-335

Van den Bosch LM, Verheul R, Schippers GM, von den Brink W (2002): Dialectical Behaviour Therapy for borderline patients with an without substance use problems. Implementation and long-term effects. Addict Behav 27 (6), 911-923

Van Loey NE, Maas CJ, Faber AW, Taal LA (2003): predictors of chronic posttraumatic stress symptoms following burn injury: results of a longitudinal study. $J$ Trauma Stress $\underline{16}$ (4), 361-369

Vollmer HC (1988): Die vorzeitige Therapiebeendigung bei der Entwöhnungsbehandlung Drogenabhängiger: Analyse und Interventionen. Suchtgefahren 34, 6579

Vollmer HC, Ellgring H (1988): Die Vorhersage der vorzeitigen Therapiebeendigung bei Drogenabhängigen. Suchtgefahren $\underline{34}$ (4), 273-284

Vogt M, Küfner H, Weiler D (2000): Katamnestische Ergebnisse zur ambulanten medizinischen Rehabilitation unter Methadon-Substitution. Suchtmed Forsch Prax 2, 93-102

Wänke M: CIP. Was mit Werbung in den Köpfen der Konsumenten geschieht; in: Werbung, Strategien und Konzepte für die Zukunft; hrsg. v. Mattenklott A, Schimanski A; Vahlen, München 2002, 482-503

Walter D A, Rosenquist P B, Bawtinhimer G (2004): Distance learning technologies in the training of psychiatry residents: a critical assessment. Acad. Psychiatry $\underline{28}$ (1), 60-65

Watson M, Pettingale K W, Goldstein D (1983): Effects of fear appeal and arousal, self-reported anxiety, and attitude towards smoking. Psychol Rep $\underline{52}$ (1), 139-146 
Weinstein N D (1980): Unrealistic optimism about future life events. J Pers Soc Psychol $\underline{39}$, 806-820

Weinstein N D (1984): Why it won't happen to me. Perception of risk factors and suspectibility. Health Psychol $\underline{3}$ (5), 431-457

Wild A, Esse S, Schulz W: Evaluierung des qualifizierten Drogenentzugs im Niedersächsischen Landeskrankenhaus Lüneburg; in: Schriftenreihe der Suchtforschungsstelle Ostniedersachsen; Heft 25; hrsg. V. Mauthe J-H, Schulz W, Braunschweig 1995, 1-75

Wilsnack SC, Vogeltanz ND, Klassen AD, Harris TR (1997): Childhood sexual abuse and women`s substance abuse: national survey findings. J Stud Alcohol $\underline{58}$, 264-271

Wittchen H U, Essau C A, von Zerssen D, Krieg J C, Zaudig M (1992): Lifetime and six-month prevalence of mental disorders in the Munich Follow-up Study. Eur Arch Psychiatry Clin Neurosci 241, 247-258

Witte K, Allen M (2000): A meta-analysis of fear appeals: implications for effective public health campaigns. Health Educ Behav 27 (5), 591-615

Wöller W (2006): Störung der Emotionsregulierung bei komplexen Traumafolgestörungen. Nervenarzt $\underline{77}, 327-332$

Ziedonis DM, Kosten TR (1991) Depression as a prognostic factor for pharmacological treatment of cocaine dependence. Psychopharmacol Bull 27 (3), 337-343

Zumbeck S, Teegen F, Dahme B, Farley M (2003): Posttraumatische Belastungsstörungen bei prostituierten. Ergebnisse einer Hamburger Studie im Rahmen eines Internationalen Projekts. Z Klein Psychol Psychiatr Psychother 2 (51), 121136 


\section{Danksagung}

Ich danke Herrn Prof. Dr. med. Wolfgang Poser für sein kontinuierliches Interesse und seine anregende Unterstützung meiner wissenschaftlichen Tätigkeit. Er hatte jederzeit ein offenes Ohr für meine Fragen und stand mir mit wertvollen Hinweisen zur Seite.

Meine klinische Lehrerin Christel Lüdecke ist mir durch ihre umfangreiche und hilfreich - kritische Förderung eine Stütze meiner Tätigkeit. Ich danke ihr für ihre immer gewährte freundliche Ermutigung, Unterstützung sowie fruchtbare Diskussion während der Arbeit an meiner Dissertation.

Des Weiteren danke ich Herrn Dr. med. M. Koller, der mich in seiner Funktion als Ärztlicher Direktor des Niedersächsischen Landeskrankenhauses Göttingen bzw. des Asklepios Fachklinikums Göttingen unterstützte.

Außerdem gilt besonderer Dank den Kolleginnen und Kollegen, den Schwestern und Pflegern, den Patientinnen und Patienten der Entgiftungsstationen des ehemaligen Niedersächsischen Landeskrankenhauses Göttingen bzw. des Asklepios Fachklinikums Göttingen, die alle auf verschiedene Art hilfreich zum Gelingen dieser Arbeit beigetragen haben.

Für Beratung in statistischen Fragen und kritische Anregungen danke ich Frau Dipl.-Psych. Stefanie Müller, für Unterstützung bei der Erledigung der Schreibarbeiten danke ich Frau Ingrid Groener und Frau Helga Böker und für die kontinuierliche Ermunterung, diese Arbeit fortzusetzen, Herrn Prof. Dr. H. Friedrich. 


\section{Lebenslauf}

Ich wurde am 09.10.1964 in Lüchow geboren. Von 1970 bis 1974 besuchte ich die Grundschulen in Müssingen und Schnega. Meine gymnasiale Ausbildung, die von 1974 bis 1983 am Gymnasium Lüchow stattfand, beendete ich 1983 mit dem Abitur.

Von 1984 bis 1991 studierte ich Humanmedizin an der Medizinischen Fakultät der Georg-August-Universität Göttingen. Am 23.10.1991 schloss ich mit dem 3. Abschnitt der ärztlichen Prüfung das Staatsexamen an der Medizinischen Fakultät der Universität Göttingen ab.

Von 1992 bis 1994 begann ich meine psychiatrische und psychotherapeutische Weiterbildung als Ärztin im Praktikum an der Psychiatrischen Klinik der Universität Göttingen (Chefarzt: Prof. Dr. med. E. Rüther) und setzte diese von 1995 bis 1998 als Assistenzärztin im ehemaligen Niedersächsischen Landeskrankenhaus Tiefenbrunn (Ärztlicher Direktor: Prof. Dr. med. MA U. Streeck) fort. 2003 erlangte ich die Zusatzbezeichnung „Psychotherapie“ und 2005 die Zusatzbezeichnung „Psychoanalyse". Meine psychoanalytische Ausbildung habe ich berufsbegleitend am Lou-Andreas-Salomé-Institut Göttingen absolviert.

Von 1998 bis 1999 war ich im Rahmen meiner neurologischen Ausbildung in der Asklepios Klinik Schildautal für neurologische Rehabilitation in Seesen (Chefarzt: Prof. Dr. med. Holzgraefe) beschäftigt, und von 1999 bis 2000 habe ich mich internistisch in der Fachklinik am Hasenbach (Chefarzt: Dr. med. M. Winkler) weitergebildet.

Seit Sommer 2000 arbeite ich im ehemaligen Niedersächsischen Landeskrankenhaus Göttingen (jetzt Asklepios Fachklinikum Göttingen, Ärztlicher Direktor: Dr. med. M. Koller) und habe am 18.08.2005 die Anerkennung als Fachärztin für Psychiatrie und Psychotherapie erworben. Seit 2005 bin ich mit dem Musiker Christoph Busse verheiratet. Nach der Geburt unserer Tochter 2008 und einer einjährigen Pause durch Elternzeit bin ich seit 2009 als Oberärztin im Asklepios Fachklinikum Göttingen im Bereich Allgemeinpsychiatrie, Schwerpunkt Sucht (Chefärztin: Christel Lüdecke) tätig. 
